\title{
Analysis of hybrid-ARQ based relaying protocols under modulation constraints
}

Tarik K. Ghanim

West Virginia University

Follow this and additional works at: https://researchrepository.wvu.edu/etd

\section{Recommended Citation}

Ghanim, Tarik K., "Analysis of hybrid-ARQ based relaying protocols under modulation constraints" (2006). Graduate Theses, Dissertations, and Problem Reports. 1701.

https://researchrepository.wvu.edu/etd/1701

This Thesis is protected by copyright and/or related rights. It has been brought to you by the The Research Repository @ WVU with permission from the rights-holder(s). You are free to use this Thesis in any way that is permitted by the copyright and related rights legislation that applies to your use. For other uses you must obtain permission from the rights-holder(s) directly, unless additional rights are indicated by a Creative Commons license in the record and/ or on the work itself. This Thesis has been accepted for inclusion in WVU Graduate Theses, Dissertations, and Problem Reports collection by an authorized administrator of The Research Repository @ WVU. For more information, please contact researchrepository@mail.wvu.edu. 


\title{
Analysis of Hybrid-ARQ Based Relaying Protocols under Modulation Constraints
}

\author{
by \\ Tarik K Ghanim \\ Thesis submitted to the \\ College of Engineering and Mineral Resources \\ at West Virginia University \\ in partial fulfillment of the requirements \\ for the degree of \\ Master of Science \\ in \\ Electrical Engineering \\ Matthew C. Valenti, Ph.D., Chair \\ Daryl Reynolds, Ph.D. \\ Brian Woerner, Ph.D.
}

Lane Department of Computer Science and Electrical Engineering

Morgantown, West Virginia

2006

Keywords: Capacity, Hybrid-ARQ, Information Outage Probability

Copyright 2006 Tarik K Ghanim 


\begin{abstract}
Analysis of Hybrid-ARQ Based Relaying Protocols under Modulation Constraints

by

Tarik K Ghanim
\end{abstract}

In a seminal paper published in 2001, Caire and Tuninetti derived an information theoretic bound on the throughput of hybrid-ARQ in the presence of block fading. However, the results placed no constraints on the modulation used, and therefore the input to the channel was Gaussian. The purpose of this thesis is to investigate the impact of modulation constraints on the throughput of hybrid-ARQ in a block fading environment. First, we consider the impact of modulation constraints on information outage probability for a block fading channel with a fixed length codeword. Then, we consider the effect of modulation constraints upon the throughput of hybrid-ARQ, where the rate of the codeword varies depending on the instantaneous channel conditions. These theoretical bounds are compared against the simulated performance of HSDPA, a newly standardized hybrid-ARQ protocol that uses QPSK and 16-QAM bit interleaved turbo-coded modulation. The results indicate how much of the difference between HSDPA and the previous unconstrained modulation bound is due to the use of the turbo-code and how much is due to the modulation constraints. The same analysis is carried out on systems using hybrid-ARQ in practical relay networks as discussed by a paper by Zhao and Valenti that was published in 2005, where retransmissions can occur from relays that have overheard the source and decoded the message successfully. Then again, as in Caire's paper, Zhao's did not place any constraints upon modulation when presenting an information theoretic bound on the performance limits of multi-terminal relay networks in a block fading channel. Thus, further investigation into the impact of modulation constraints on the use of hybrid-ARQ with practical relay networks will be considered in this thesis, in contrast to the conventional hybrid-ARQ mentioned earlier. Performance limits and numerical results are presented for some representative topologies of practical relay networks. 


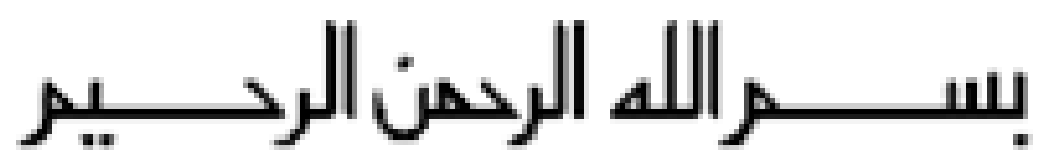

To my father, for his endless love and support and to my mother, for her patience and unconditional love. To my great brothers, for providing all the support and guidance that one needs to succeed in life. Finally, to the greatest blessing in my family, my beautiful sister who has always been there for me. In gratitude to all the sacrifices that my family has endured and their remarkable support to all my efforts in my achievements today, I dedicate this thesis. 


\section{Acknowledgments}

No work is ever the product of one person's efforts, and certainly this thesis is no exception. Without the expert guidance, support, and genuine help from my dedicated advisor, Dr. Matthew C. Valenti, this thesis would not be possible. Dr. Valenti has helped me develop in every possible way, I am really thankful to him for being an outstanding mentor and a great friend.

Special Thanks to Dr. Daryl Reynolds and Dr. Brian Woerner for being on my committee. As well for their expert knowledge and advice which they bestowed upon me.

Completing my thesis would not be possible without the technical help of my colleagues at the Wireless Communication Research Lab, to whom I am greatly indebted.

Last but not least, my appreciation goes out to the entire LDCSEE staff, students, and all my friends in Morgantown and around the states for adding depth to my graduate program. 


\section{Contents}

Acknowledgments $\quad$ iv

List of Figures vii

$\begin{array}{ll}\text { List of Tables } & \text { ix }\end{array}$

Notation $\quad$ x

1 Introduction $\quad 1$

1.1 Motivation and Objectives . . . . . . . . . . . . . . . 1

1.2 Thesis Outline . . . . . . . . . . . . . . . . . . . 4

2 Wireless Point-to-Point Communication Systems $\quad 6$

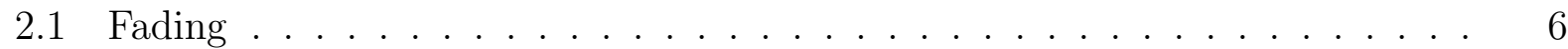

2.1.1 Large Scale Effects . . . . . . . . . . . . . . . . . . . . 7

2.1.2 Small Scale Fading . . . . . . . . . . . . . . . . . . . . . . . . 7

2.1 .3 Block Fading . . . . . . . . . . . . . . . . . . . . 10

2.2 Modulation . . . . . . . . . . . . . . . . . . . . . 10

2.2.1 Constellation Diagrams . . . . . . . . . . . . . . . . . . . . . . 12

2.2 .2 Symbol Mapping . . . . . . . . . . . . . . . . . . . . . . . 14

2.2 .3 Coded Modulation . . . . . . . . . . . . . . . . . . . 16

$2.2 .4 \mathrm{BICM} \ldots \ldots \ldots \ldots \ldots \ldots \ldots \ldots$

2.3 System Model . . . . . . . . . . . . . . . . . . . . . 17

2.4 Conclusion . . . . . . . . . . . . . . . . . . . . . . 20

3 The Information Theory of Block Fading Channels 21

3.1 Information Theory: Basic Definitions . . . . . . . . . . . . . . . . 21

3.2 Ergodic Capacity . . . . . . . . . . . . . . . . . . 23

3.2 .1 AWGN Capacity . . . . . . . . . . . . . . . . . 23

3.2.2 Ergodic Rayleigh Fading Channel Capacity . . . . . . . . . . . . . . 25

3.3 Capacity Considerations in a Block Fading Channel . . . . . . . . . . . . . 28

3.4 Conclusion . . . . . . . . . . . . . . . . . . . . . 34

4 Hybrid Automatic Repeat Request 35

4.1 Introduction . . . . . . . . . . . . . . . . . . . . 35

4.2 Hybrid-ARQ . . . . . . . . . . . . . . . . . . . 36 
4.3 Throughput . . . . . . . . . . . . . . . . . . . . . . . . 39

4.4 Numerical Results . . . . . . . . . . . . . . . . . . . . . . . . . . . . . . . . . . . . . . 40

4.5 Conclusion . . . . . . . . . . . . . . . . . . . . 43

5 Channel Coding $\quad 45$

5.1 Introduction . . . . . . . . . . . . . . . . . . . . 45

5.1 .1 Block Codes . . . . . . . . . . . . . . . . . 45

5.1 .2 Convolutional Codes . . . . . . . . . . . . . . . . 46

5.1 .3 Turbo Codes . . . . . . . . . . . . . . . . . . 48

5.2 Limits on the Throughput of HSDPA . . . . . . . . . . . . . . 51

5.3 Conclusion . . . . . . . . . . . . . . . . . . . . . 54

6 Hybrid-ARQ Based Relay Networks $\quad 56$

6.1 Introduction . . . . . . . . . . . . . . . . . . 56

6.2 Relay Network Model . . . . . . . . . . . . . . . . . . . . 58

6.3 Throughput Analysis . . . . . . . . . . . . . . . . . . . 61

6.3.1 Path Loss Exponent Coefficient . . . . . . . . . . . . . . . . . 61

6.3.2 Maximum number of Transmission, $B_{\max } \ldots \ldots \ldots . \ldots . \ldots 62$

6.3.3 Relay Network Topology . . . . . . . . . . . . . . . . . . . 64

6.4 Conclusion . . . . . . . . . . . . . . . . . . . . 67

7 Conclusions $\quad 69$

7.1 Summary and Conclusions . . . . . . . . . . . . . . . . . . . . . . . . . . . . . . 69

7.2 Future Work . . . . . . . . . . . . . . . . . . 70

$\begin{array}{ll}\text { References } & \mathbf{7 1}\end{array}$

A Numerical Integration : Information Outage Probability . . . . . . . . . . . 74

B Maximum Throughput Performance of H-Set 1 . . . . . . . . . . . . . 78 


\section{List of Figures}

2.1 Signal parameters that modulation modifies . . . . . . . . . . . . . . . . 11

2.2 I-Q Polar to Rectangular Conversion . . . . . . . . . . . . . . . . . . . . . . 12

2.3 I-Q Format: (a) BPSK (b) QPSK (c) 16PSK (d) 16QAM . . . . . . . . . . . 13

2.4 16QAM signal set with (a) gray-labeling (b) set-partioning labeling . . . . . 15

2.58 -PSK Set Partitioning . . . . . . . . . . . . . . . . 16

2.6 System model. $\pi$ denotes interleaving at the bit level. . . . . . . . . . . . . . 18

3.1 System model for input signal $X$ and output signal $Y$ in an AWGN channel with Complex Gaussian noise $\boldsymbol{\nu} \ldots \ldots$. . . . . . . . . . . . .

3.2 Capacity of QPSK, 16-QAM, and unconstrained (Gaussian-input) modulation in AWGN. For 16-QAM, the CM capacity is shown as is the BICM capacities for two types of symbol mappings (gray-labelling and set-partitioning (SP)).

3.3 Capacity of QPSK, 16-QAM, and unconstrained (Gaussian-input) modulation in an ergodic Rayleigh fading channel. For 16-QAM, the CM capacity is shown as is the BICM capacities for two types of symbol mappings (gray-labelling and set-partitioning (SP)). The unconstrained modulation capacity curve for AWGN is also shown for comparison. . . . . . . . . . . . . . . . .

3.4 Information outage probability vs. SNR for unconstrained Gaussian input modulation in Rayleigh block fading with a per block rate $R_{B}=1 / 3$ and $B=2,4$ using both code and diversity combining. . . . . . . . . . . .

3.5 Information outage probability vs. SNR for unconstrained and gray-labelled 16QAM modulation in Rayleigh block fading with rate $R_{B}=2$.

3.6 Information outage probability vs. SNR for unconstrained (solid line) and QPSK modulation (dashed line) in Rayleigh block fading for $B=1,2,3,4$ using code combining with different rates; $R_{B}=1.5,1.75$, and $2 \ldots \ldots$.

3.7 Diversity vs. number of block for the unconstrained Gaussian input (solid black), QPSK constrained modulation (dashed line), and 16 QAM constrained modulation(solid line) for different rates; $R_{B}=1.25$ (circle), 1.5 (square), and 1.75 (star) . . . . . . . . . . . . . . . . .

4.1 Information outage probability vs SNR in Rayleigh block fading using QPSK modulation with rate $R=3202 / 2400$. Unconstrained (solid line) and modulationconstrained (dashed line) theoretical limits are compared against the simulated performance of the HSDPA system (dotted line). . . . . . . . . . . . 
4.2 Throughput efficiency of hybrid-ARQ with code combining in Rayleigh block fading using QPSK or 16-QAM modulation. For each modulation type, the unconstrained and modulation-constrained theoretical limits are shown. . . .

4.3 Throughput efficiency vs. SNR for unconstrained (solid line) and QPSK modulation (dashed line) in Rayleigh block fading using different rates; $R=1.25$,

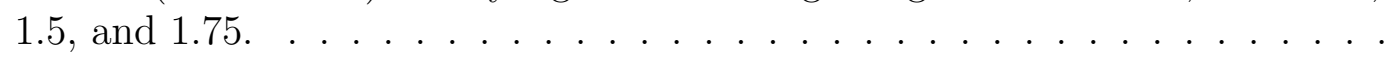

5.1 UMTS Turbo Encoder . . . . . . . . . . . . . . . . . . .

5.2 A trellis for a 4 state convolutional code. A solid line denotes a transition due to a binary input symbol 0 , where the dashed line represents the transition due to a binary input symbol $1 \ldots \ldots \ldots$

5.3 UMTS Turbo Decoder . . . . . . . . . . . . . . . . . . . .

5.4 Simulation results for a UMTS rate $\frac{1}{3}$ Turbo code in AWGN (solid line) and Rayleigh fading (dashed line) for gray-labeled 16QAM signal with $\mathrm{K}=5114$ after 14 iterations using the log-map algorithm (a) BER (b) FER . . . . . .

5.5 Throughput efficiency of HSDPA H-Sets 1 through 3 in Rayleigh block fading using QPSK or 16-QAM modulation. For each modulation type, the unconstrained and modulation-constrained theoretical limits are compared against the simulated performance of the HSDPA system. . . . . . . . . . . . . . .

6.1 A simple relay network f . . . . . . . . . . . . . . . . 58

6.2 A cluster of $\mathrm{K}$ nodes constituting a relay network . . . . . . . . . . . . .

6.3 Throughput efficiency vs. SNR for unconstrained (solid line) and QPSK modulation (dashed line) in Rayleigh block fading using different values for the path loss exponent; $\alpha=2,3,4, K_{r}=1 \ldots \ldots \ldots$

6.4 Throughput efficiency vs. SNR for unconstrained (solid line) and QPSK modulation (dashed line) in Rayleigh block fading using different values for the maximum number of transmission; $B_{\max }=2,4,6,8,10, K_{r}=1 \ldots \ldots$.

6.5 Throughput efficiency vs. SNR for unconstrained (solid line) and QPSK modulation (dashed line) in Rayleigh block fading for different relative relay distances, $K_{r}=1$. (a) Relay closer to source (b) Relay closer to destination . .

6.6 Throughput efficiency vs. SNR for unconstrained (solid line) and QPSK modulation (dashed line) in Rayleigh block fading using different number of relays; $K_{r}=1,2,3,5,10$ in an equidistant linear topology $\ldots \ldots \ldots$. . . . .

.1 Information outage probability vs SNR in Rayleigh block fading for the unconstrained Gaussian input with rate $R=2$. Numerical integration results (solid line) and Monte Carlo results (star). . . . . . . . . . . . . . .

.2 Maximum Throughput of HSDPA H-Set 1 in Rayleigh block fading using QPSK (black) or 16-QAM (red) modulation. For each modulation type, the unconstrained (solid line) and modulation-constrained (dashed line) theoretical limits are compared against the simulated performance of the HSDPA system (dotted line). 


\section{List of Tables}

4.1 Value of normalized throughput at 0.5 for different rates . . . . . . . . . . 42

5.1 Comparison of turbo code performance against AWGN and ergodic Rayleigh channel capacity $\left(B E R=10^{-5}\right)$ at rate $\frac{1}{3}$ in terms of $\frac{E_{b}}{N_{o}}$ under modulation constraints . . . . . . . . . . . . . . . . . . 51

5.2 Maximum Throughput (kbps) for the Fixed Reference Channel Definitions:

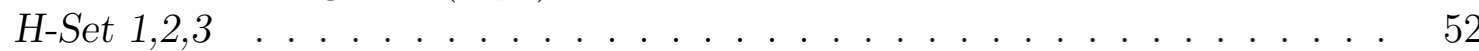




\section{Notation}

We use the following notation and symbols throughout this thesis.

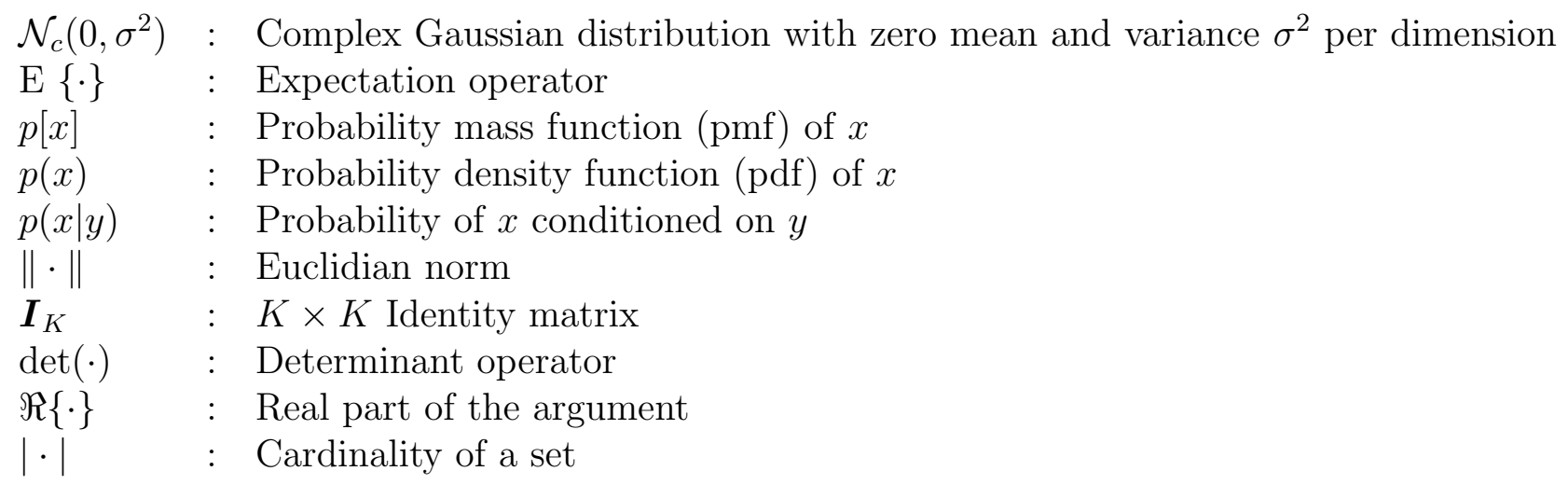

Bold uppercase letters denote matrices while bold lower case letters denote vectors. 


\section{Chapter 1}

\section{Introduction}

\subsection{Motivation and Objectives}

With the emergence of many portable devices on the market (e.g. laptops, personal digital assistants PDAs, hand-held phones...etc) that use wireless communications to interact with each other and the wireless network, reliance on battery life for these wireless devices' operation has become a major concern. Hence, the design of wireless communication protocols must be done very carefully and make prudent use of the available energy. It, therefore, makes sense to look for alternative protocol strategies focused on power savings and energy management. Instead of trying to provide the device with more power, one should try to build devices that can perform the same function and provide the same services while reducing the overall power consumption. Given the power limitation, a growing pool of wireless applications and protocols have been deployed and many others are still being developed. One of the many ongoing ventures is the IEEE 802.15.4 or Low Rate Wireless Personal Area Network (LRWPAN) [1], which has been the offspring of the success of wireless local area networks (WLANs). LRWPAN has been focused on enhancing the capabilities of WLANs to meet the emerging need for energy efficient wireless systems by providing a standard that uses extremely low-power for wireless connectivity among inexpensive, fixed, portable and moving devices. Another approach for power conservation is to choose energy efficient modulation and error control schemes that focus on the energy needs of the system. This thesis analyzes the hybrid Automatic Repeat reQuest (hybrid-ARQ) protocol that addresses 
this challenge of energy efficiency in the wireless environment while maintaining the desired throughput and error performance.

In any wireless communication system, there is always a need to deal with the high error rates arising from the complex and varying channel conditions. Two ways to control such errors are retransmission and error correction. Computer communication generally implements a reliable data transfer using either methods or a combination of them at different levels in the protocol stack. Forward error correcting codes are mainly used at the physical layer to reduce the impact of errors in the wireless connection. In most cases, these codes provide less than perfect protection and some amount of residual errors pass through. The upper protocol layers employ various block error detection and retransmission schemes. Hybrid-ARQ is one of the more mature examples of cross-layer protocol design, combining physical layer forward error correction (FEC) with link layer ARQ [2]. With hybrid-ARQ, a message is encoded by a low rate mother code and then partitioned into several blocks. Blocks are sent one by one until enough information is accumulated at the destination to correctly decode the message. Often, the channel is uncorrelated from one block to the next, in which case a block fading model may be assumed. A key performance metric for hybrid-ARQ is its throughput, which is the number of bits conveyed per unit time. In [3], Caire and Tuninetti derived information-theoretic bounds on the throughput of hybrid-ARQ in block fading. The results built upon related work on the performance of standard block fading channels $[4,5]$, i.e. channels with a fixed code rate and number of blocks per codeword. The results in [3] placed no constraints upon modulation, and as a consequence, the input to the channel was assumed to be Gaussian distributed. However, practical systems do not use Gaussian-distributed modulation, and the computation of information-theoretic limits on the throughput of hybrid-ARQ with practical modulation constraints has until now remained an open problem. The purpose of this thesis is to investigate how modulation constraints affect the performance of block fading channels in general (an issue that has also been recently discussed in [6]), and more specifically, the throughput of hybrid-ARQ over a block fading channel. This aspect of the thesis has been published in [7].

This thesis first analyzes hybrid-ARQ over point-to-point links. One specific example that will be presented is the High Speed Downlink Packet Access (HSDPA) standard 
[8, 9], which is part of the Universal Mobile Telecommunications System (UMTS) family of standards under development by the Third Generation Partnership Project (3GPP). HSDPA is a newly standardized hybrid-ARQ protocol that uses quaternary phase shift-keying (QPSK) and (gray-labelled) 16 quadrature amplitude modulation (QAM) bit interleaved turbo-coded modulation. As shown in [10], the performance of a bit-interleaved coded modulation (BICM)-constrained system can differ significantly from that of a system with an unconstrained Gaussian input, especially at high spectral efficiency.

The second emphasis of this thesis is the application of hybrid-ARQ to practical relay networks. Fuelled by the need to enable inexpensive energy efficient wireless sensor network applications, Zhao and Valenti in [11] proposed a relaying protocol that uses hybrid-ARQ. Relay networks have recently received increasing attention as it provides flexible extension and the potential capacity increase to the traditional wireless systems. Relay networks exploit the inherent spatial diversity that is available in a wireless environment much like Multiple Input Multiple Output (MIMO) systems, where multiple transmit and receive antennas are deployed. Spatial diversity is present whenever there is uncorrelated fading due to the presence of multiple devices/antennas with spatial separation.

The relay channel was first studied by Meulen [12] and then further explored by Cover et al. [13]. More recently, cooperation among source node/relay nodes have been proposed for relay network to further utilize the spatial diversity created by the independent nodes' locations (e.g. [14] [15] [16]). This is in contrast to the traditional multi-hop protocols. In multi-hop protocols, the nodes in the wireless network are treated as a cascade of point-topoint links whereby each transmission is directed to one other node. This results in a failure to make use of the spatial diversity available in the network introduced by the broadcasting nature of wireless communication. On the other hand, relay networks use cooperative communication to exploit the broadcast nature of the wireless medium (i.e. all nodes in the network can hear the source's transmission as long as they are within broadcasting range). If an intermediate node can decode the message, it can act as a relay sending information to the destination, which the destination can combine with the information it already received from the source. Space-time signal processing techniques are utilized to enable the cooperation among nodes. Adding relays in a wireless environment reduces the signal transmission 
distances, resulting in lower propagation loss and higher average signal-noise ratio to the mobile user. This results in expending less energy when transmitting messages.

In [11], an integrated approach to wireless networks comprised of multiple relays is proposed, where the relays operate over orthogonal time slots. The main contribution of the paper was the postulation that systems using hybrid-ARQ need not send all retransmissions from the source. Instead, retransmissions can occur from relays that have overheard the source and decoded the message successfully. However, as in [3], [11] placed no constraints upon modulation when presenting an information theoretic bound on the performance limits of multi-terminal relay networks in a block fading channel. As a consequence, the transmitted symbols were assumed to be drawn from a Gaussian distribution. However, as will be discussed in Chapter 2, practical digital communication systems must use symbols drawn from a finite constellation. Thus, further investigation into the impact of modulation constraints on the use of hybrid-ARQ with practical relay networks in different topologies will be considered in this thesis.

\subsection{Thesis Outline}

The focus of this thesis is the application of hybrid-ARQ protocols with practical modulation in point-to-point links and in practical relay networks. To accomplish this goal, Chapter 2 begins with an overview of wireless communication systems. Specifically, issues relating to the wireless channel such as large scale and small scale fading and ways to mitigate them are discussed. Modulation, which is a physical layer technique that is used to convey information over the wireless channel, is also discussed. The wireless link quality is significantly improved when channel coding is combined with the modulation, whether via coded modulation or bit-interleaved coded modulation. The chapter ends with an exposition of our system model for the wireless links.

In Chapter 3, a review of the basics of information theory is presented, including a discussion of BICM-constrained capacity of simple additive white Gaussian noise (AWGN) channels. This is followed by a discussion of the information outage probability of a block fading channel with both unconstrained and constellation-constrained inputs for a fixed 
rate codeword. After introducing the types of hybrid-ARQ, Chapter 4 builds upon the results in Chapter 3 to derive the throughput and latency of hybrid-ARQ, where the rate of the codeword varies depending on the instantaneous channel conditions, under modulation constraints in block fading, thereby generalizing the results of [3]. Numerical results are used to compare the simulated throughput of the unconstrained bound of [3] and the modulationconstrained bounds developed in this thesis. These results quantify the losses due to the use of modulation constraints. Chapter 5 introduces some of the coding techniques that would approach the capacity limit discussed in Chapter 3. Numerical results are used to compare the simulated throughput of HSDPA against the unconstrained and modulation-constrained bounds. These results indicate how much of the difference between HSDPA and the previous unconstrained modulation bound is due to the use of the turbo-code and how much is due to the modulation constraints.

Chapter 6 starts by presenting an overview of relay networks and its corresponding system model. A contrast of the use of conventional hybrid-ARQ protocol used in the previous chapter to the modifications for relay networks proposed by [11] is then presented. Using the results derived earlier for throughput under modulation constraints, performance limits and numerical results are presented for some representative topologies of practical relay networks. Finally, Chapter 7 provides conclusions and suggestions for future work. 


\section{Chapter 2}

\section{Wireless Point-to-Point \\ Communication Systems}

This chapter presents a general overview of the wireless channel and the types of degradation manifested in the channel as is portrayed in [17]. That is followed by a discussion about conveying information over the wireless channel through modulation of a sinusoidal carrier. Signals that are transmitted simultaneously over the same medium without modulation would clearly interfere with each other. The chapter culminates in a mathematical model for coded communications over wireless channels.

\section{$2.1 \quad$ Fading}

Ideally, under a free-space propagation model, radio frequency (RF) energy propagating from a transmitter to a receiver attenuates according to the inverse-square law; hence the power of the received signal can be predicted with a high confidence. Practically, free-space conditions are not satisfied due to a diversity of propagation paths that cause variations in the received signal, called fading. Two types of channel effects are recognized. Fading can be further classified as either large scale or small scale. 


\subsubsection{Large Scale Effects}

The large scale effects of shadowing and path-loss refer to variations in the average pathloss due to motion over large areas. Path losses arise due to eminent terrain profiles, which tend to shadow part of the free-space propagating signal, and is basically caused by reflection, diffraction and scattering of the RF energy. Statistically, the path loss has a mean that obeys the $n$-th power law and if expressed in $\mathrm{dB}$ is:

$$
L_{d B}(d)=L_{d B}\left(d_{0}\right)+10 \alpha \log _{10}\left(d / d_{0}\right)+X_{\sigma, d B}
$$

where $L_{d B}\left(d_{0}\right)$ is the free space path-loss at a far field point $d_{0}$ away from the transmitter $\left(d_{0}\right.$ is typically $1 \mathrm{~km}$ for large cells, $100 \mathrm{~m}$ for micro cells and $1 \mathrm{~m}$ for indoor environments). $\alpha$ is the path-loss exponent and is dependent on frequency, antenna heights, and the environment. $X_{\sigma} \mathrm{dB}$ is a zero mean Gaussian random variable with a standard deviation $\sigma$ which accounts for the variation in received signal strength, called shadowing. This phenomenon is usually overcome by including a shadow margin, the $d B$ loss due to shadowing, in the budget link analysis.

\subsubsection{Small Scale Fading}

Small scale (e.g. Rayleigh) fading refers to abrupt changes in the path-loss for small changes (in the order of $\lambda / 2$ ) in the separation between the transmitter and the receiver. Small-scale fading takes place due to the time-spreading of the underlying pulses within the signal and the time variant nature of the channel due to the motion involved. The time-spreading mechanism is characterized by the power delay profile resulting from the channel's non-optimum impulse response, while the time-variant behavior of the channel is characterized by the channel autocorrelation function (or Doppler spectrum).

Statistically, if there are a large number of multiple reflection paths and no line-of-sight signal component, then the envelope of the received signal follows a Rayleigh distribution. Mathematically speaking, if we have two random variables $X$ and $Y$ that are independent and identically distributed with $\mathcal{N}_{c}\left(0, \sigma^{2}\right)$ then $R=\sqrt{X^{2}+Y^{2}}$ is a Rayleigh random variable with the following probability density function: 


$$
p_{R}(r)= \begin{cases}\frac{r}{\sigma^{2}} \exp \left[\frac{-r^{2}}{2 \sigma^{2}}\right] & \text { for } r \geq 0 \\ 0 & \text { otherwise }\end{cases}
$$

Otherwise, if there is a strong line-of-sight component, the envelope will follow a Rician distribution.

Either of the two small-scale fading mechanisms can be equivalently studied in both time-domain and frequency-domain. Bello in [18] proposed the Wide-Sense Stationary Uncorrelated Scattering model (WSSUS) to study the small-scale fading phenomenon. In this model, the impulse response of the channel - viewed in the delay-time domain $(\tau)$ as the multi-path intensity profile - deviates from the ideal channel response because of the several discrete multi-path components of the received signal. A figure of merit is the maximum excess delay $\left(T_{m}\right)$, which is the time between the first and the last arrivals of the diverse signal components. A more useful parameter is the root mean squared delay spread $\sigma_{\tau}$. According to the relation between $T_{m}$ and the symbol time, $T_{s}$ (pulse width), two degradation categories are recognized:

1. Frequency-Selective fading $\left(T_{m}>T_{s}\right)$, which causes a channel induced ISI

2. Flat fading $\left(T_{m}<T_{s}\right)$, which causes the SNR to fluctuate since the unresolvable phasor components can add up constructively or destructively.

Flat fading can be mitigated by increasing the received SNR (in digital systems, signal diversity and error-correction coding are employed as an alternative to increasing received SNR). Analogous characteristics of the time-spreading mechanism can be viewed in the frequency domain using the spread-frequency correlation function, which is the Fourier transform of the multi-path intensity profile, and represents the correlation between the channel's response to two signals as a function of the frequency difference between them. A figure of merit in this case is the coherence bandwidth $\left(f_{0}=1 / T_{m}\right)$, which is a measure of the range of frequencies over which the channel passes all spectral components with approximately equal gain and linear phase. According to the relation between $f_{0}$ and the symbol rate $\left(W=1 / T_{S}\right)$, 
a channel may exhibit a frequency-selective fading $\left(f_{0}<W\right)$ or a flat fading $\left(f_{0}>W\right)$. However, there are instances when a channel undergoes frequency-selective fading despite having $\left(f_{0}>W\right)$ owing to the occasional loss of the base band DC component of the received signal [17]. This effect is less likely to occur when $f_{0}$ is much larger than $W$.

The relative motion between the receiver and the transmitter causes a time-variant channel behavior. In the time-domain, this effect is studied through the Spaced-time Correlation function, which is the auto-correlation function of the channel's response to a sinusoid at different excitation times. This approach introduces the coherence time $\left(T_{0}\right)$, which measures the expected time duration over which the channel's response is essentially invariant. According to the relation between $T_{0}$ and $T_{s}$, two degradation categories are recognized:

1. Fast fading $\left(T_{0}<T_{s}\right)$, which implies that the channel fading is likely to change several times over a symbol period. This in turn results in synchronization problems due to pulse distortion.

2. Slow fading $\left(T_{0}>T_{s}\right)$, which implies that the channel is expected to maintain a virtually constant fading level during each symbol propagation period. Hence, pulse distortion is unlikely to take place and the degradation is mostly due to the fading effect.

The time-variant nature of a mobile RF channel can also be viewed in the frequency domain using the Doppler power spectral density function, which is the Fourier transform of the spread-time correlation function. A figure of merit for this approach is the spectral broadening (Doppler Spread) $\left(f_{d}=1 / T_{0}\right)$. The time-variant nature can be interpreted as a result of the Doppler shift of frequency in the multi-path components of the received signal, which causes frequency broadening of the received signal. The Doppler spread defines the fading rate of the channel $\left(f_{d}<W\right)$ resulting in a slow fading channel, and $\left(f_{d}>W\right)$ in a fast fading channel. The spectral broadening effect due to the time-variant nature of the channel is analogous to that caused by digital signal keying [17]. It is desirable to have $f_{d}<<W$ in order to avoid the random frequency modulation of the received signal because of the varying Doppler shifts. 


\subsubsection{Block Fading}

In order to model slow frequency nonselective fading wireless channels, [5] introduced the block fading channel. Irrespective of the block length, blocks in this channel experience constant fading for the whole duration of a block, where the fading coefficients are independent from one block to the next. With block fading, the channel's coherence time, $T_{0}$, is larger than the symbol time $T_{s}$. This means that each codeword experiences only one realization of the propagation channel. Henceforth, no codeword in a block fading channel can span all the states of the channel, or in a sense average the channel effect thereby removing any ergodicity notion for the channel.

The block fading model with its simplistic assumptions is a good approximation for slow fading channels, thereby providing great means for many wireless communications. Some examples that are well-modelled by block fading include frequency hopping and hybrid-ARQ. Current standards using these examples include Global System for Mobile Communications (GSM), Enhanced Data GSM Environment (EDGE), and High Speed Downlink Packet Access (HSDPA).

\subsection{Modulation}

Modulation is the process of mapping digital information onto analog waveforms that match the characteristics of the channel. Modulation schemes are distinguished by their bandwidth efficiency, energy efficiency, and complexity (or cost efficiency). Bandwidth efficiency describes the ability of a modulation scheme to accommodate data within a limited bandwidth. Energy efficiency describes the ability of the system to reliably send information at the lowest practical power level. The parameter to be optimized depends on the demands of the particular system, for example, designers of hand-held cellular phones put a high priority on energy efficiency because these phones need to run on a battery, while bandwidth efficiency is important for wireline systems like telephone modems. Cost is also a high priority because cellular phones must be low-cost to encourage more users. Accordingly, these systems sacrifice some bandwidth efficiency to get energy and cost efficiency. Given 
Amplitude

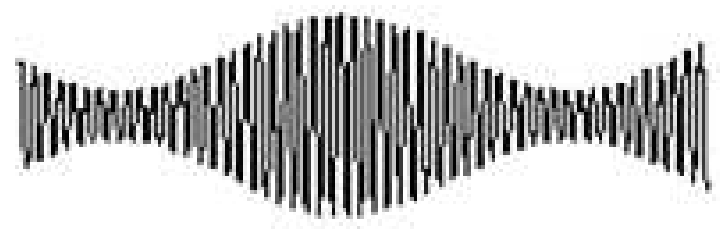

Frequency

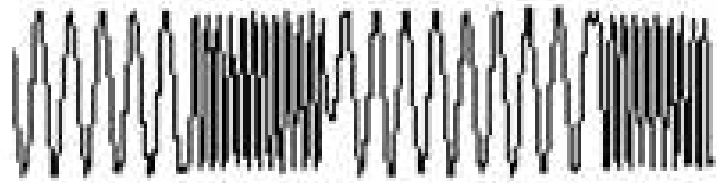

Phase

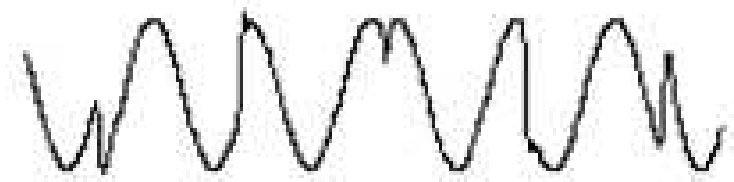

\section{Amplitude \& Phase}

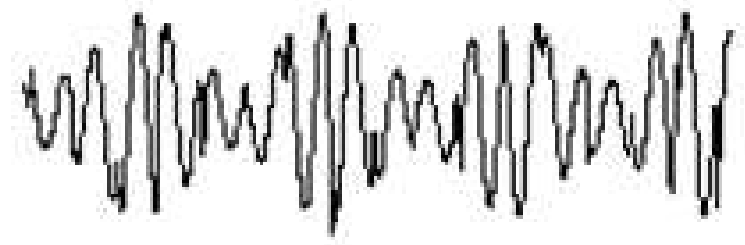

Figure 2.1: Signal parameters that modulation modifies

the tradeoffs that must be considered in digital RF communications design, this section will further elaborate on how information is modulated onto in-phase $(I)$ and quadrature $(Q)$ signals and will discuss the different types of digital modulation that are used throughout this thesis.

Modulated signals can convey information through varying three parameters of the bandpass signal $v(t)=R(t) \cos \left(2 \pi f_{c} t+\theta(t)\right)$ that has a bandwidth $W(\mathrm{~Hz})$ and is centered at $f_{c}$ $(\mathrm{Hz})$. Amplitude Shift Keying (ASK) conveys information by varying the amplitude $R(t)$ of the input signal. Phase Shift Keying (PSK) varies the phase $\theta(t)$, Frequency Shift Keying (FSK) varies the instantaneous frequency at which the signal is centered about to modulate information. Quadrature Amplitude Modulation (QAM) uses a combination of ASK and PSK to modulate signals, whereby the information is conveyed by varying the amplitude and the phase of the baseband signal.

Fig. 2.1 illustrates the modulation techniques. ASK represents different bits by simply changing the amplitude of the transmitted signal. The idea behind PSK is to have the signals 
containing different phase values correspond to the different sets of data bits. FSK transmits signals at different frequencies depending on the value of the data bits. QAM, on the other hand, combines both phase and amplitude modulation.

\subsubsection{Constellation Diagrams}

Linear modulation formats, like ASK, PSK, and QAM, are often expressed in terms of in-phase (I) and quadrature (Q) components. I and Q present a rectangular representation of the polar diagram, where the I axis lies on the zero degree phase reference, and the $\mathrm{Q}$ axis is rotated by 90 degrees. The signal vector's projection onto the I axis is its in-phase component and the projection onto the $\mathrm{Q}$ axis is its quadrature component, as shown in Fig. 2.2 and the following equation:

$$
v(t)=x_{I}(t) \cos \left(2 \pi f_{c} t\right)-x_{Q}(t) \sin \left(2 \pi f_{c} t\right)
$$

Digital modulation maps the data to a number of discrete points on the I/Q plane. These are known as constellation points.

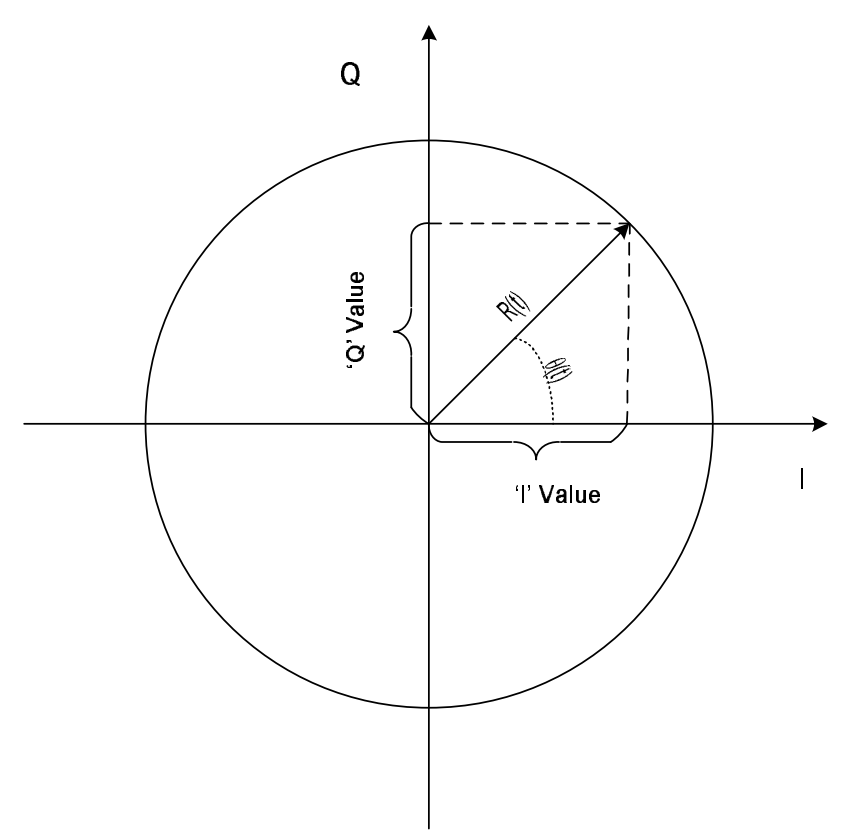

Figure 2.2: I-Q Polar to Rectangular Conversion 


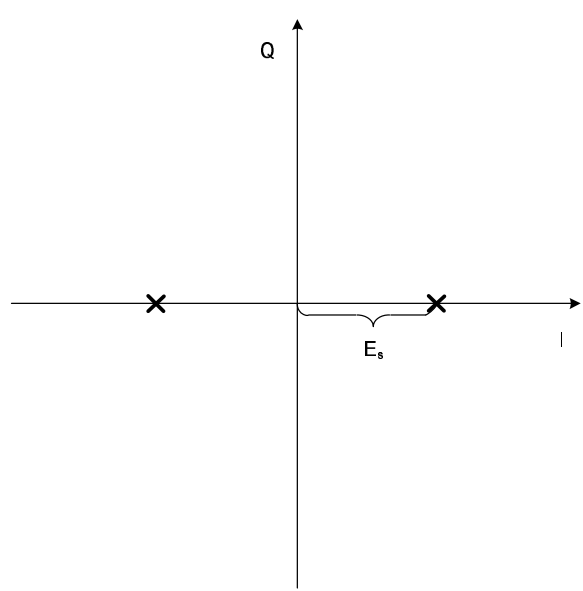

(a)

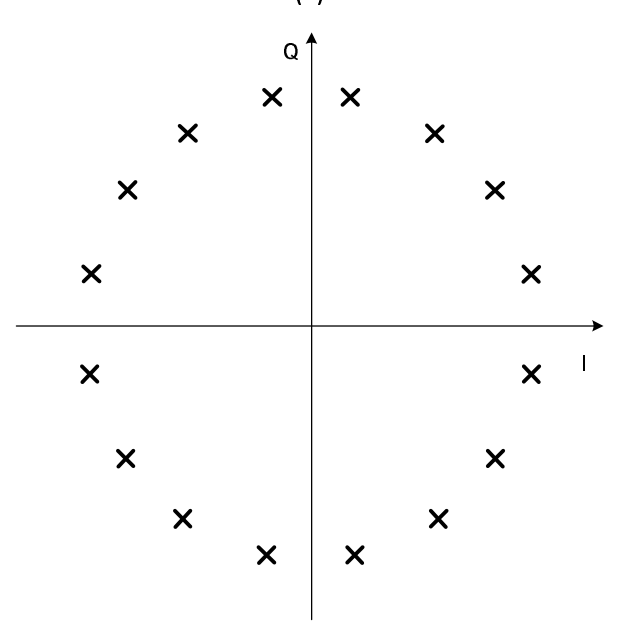

(c)

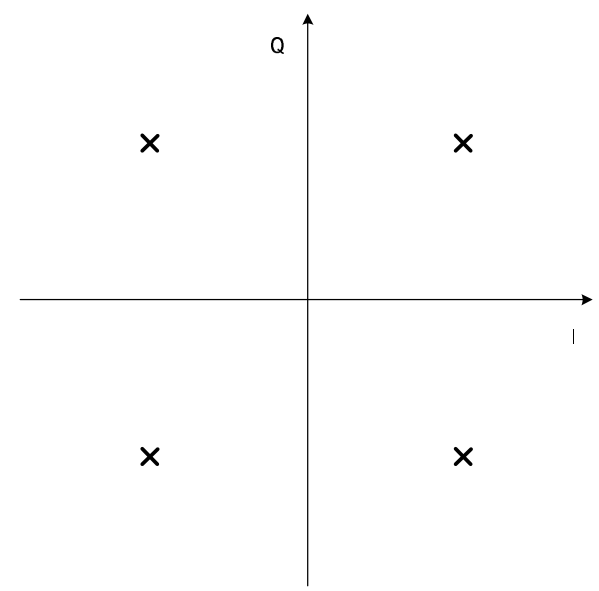

(b)

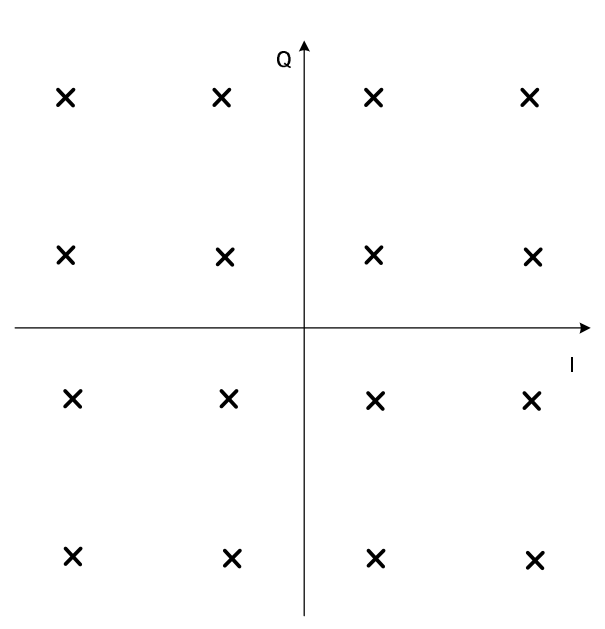

(d)

Figure 2.3: I-Q Format: (a) BPSK (b) QPSK (c) 16PSK (d) 16QAM

For example, Fig. 2.3 represents the signal constellations of Binary PSK (BPSK) and of Quadrature PSK (QPSK) in the I/Q plane. Note that the distance from the origin represents the energy of the signal $\mathcal{E}_{x}=\|\mathbf{x}\|^{2}$, also called the squared norm of $\mathbf{x}$.

To understand and compare different modulation format efficiencies, it is important to first understand the difference between bit rate and symbol rate. The signal bandwidth for the communications channel depends on the symbol rate $\left(R_{s}\right)$, not on the bit rate $\left(R_{b}\right)$. Assume that we have a finite signal constellation to be used for transmission. Define $M \triangleq|\mathcal{S}|$ to equal the number of signals in the signal set $\mathcal{S}$. Then for every signal $x \in \mathcal{S}$ there exists an $m$-tuple of binary data associated with it, where $m=\log _{2} M$. In order to represent $M$ 
signals with binary information, one needs $2^{m}$ bits. ${ }^{1}$ Hence,

$$
R_{s}=\frac{R_{b}}{m}
$$

A very simple example illustrating the efficiency of a modulation format is the difference between BPSK and QPSK, as shown in Fig. 2.3(a)(b), respectively. In BPSK, there are two constellation points and thus we need only $2^{1}$ or $m=1$ bit to represent the signals. On the other hand, we need $2^{2}$ or $m=2$ bits to represent the four QPSK symbols. Therefore, we can transmit 2 bits instead of 1 for each transmission while still occupying the same bandwidth. From equation (2.4), we can see that QPSK provides twice the bit rate when QPSK symbols are transmitted, thereby increasing the bandwidth efficiency.

Different modulation formats can have different energy efficient implementations. The key issue influencing energy efficiency is the spacing between the signals. Fig. 2.3(c)(d) illustrates 16PSK and 16QAM constellation diagrams. Both of these modulations have the same bandwidth efficiency since every point is represented by 4 bits. However, they have different energy efficiencies. The probability of mistaking one signal for another is related to the distance between signal points. It can be clearly seen that Fig. 2.3(d) has widely spaced signals which will result in lower error rates than the tightly packed signals in Fig. 2.3(c).

\subsubsection{Symbol Mapping}

Mapping, or labeling, is how bits are mapped to signals. Labeling affects the bit error rate (BER) by determining how many bit errors occur each time there is a symbol error. Fig. 2.4 shows the constellation diagram for 16 QAM with two different labelings, namely, gray and set-partitioning labeling. A gray labeling assigns to each set of neighboring symbols a vector of bits that differ by one bit only. This scheme is also known as single-distance labeling, reflecting the Hamming distance of 1 between adjacent symbols, where Hamming distance is defined as the number of positions that two symbols differ, denoted as

$$
d_{H}(\mathbf{v}, \mathbf{w})=w(\mathbf{v}+\mathbf{w})
$$

\footnotetext{
${ }^{1}$ Typically $M$ is a power of 2.
} 


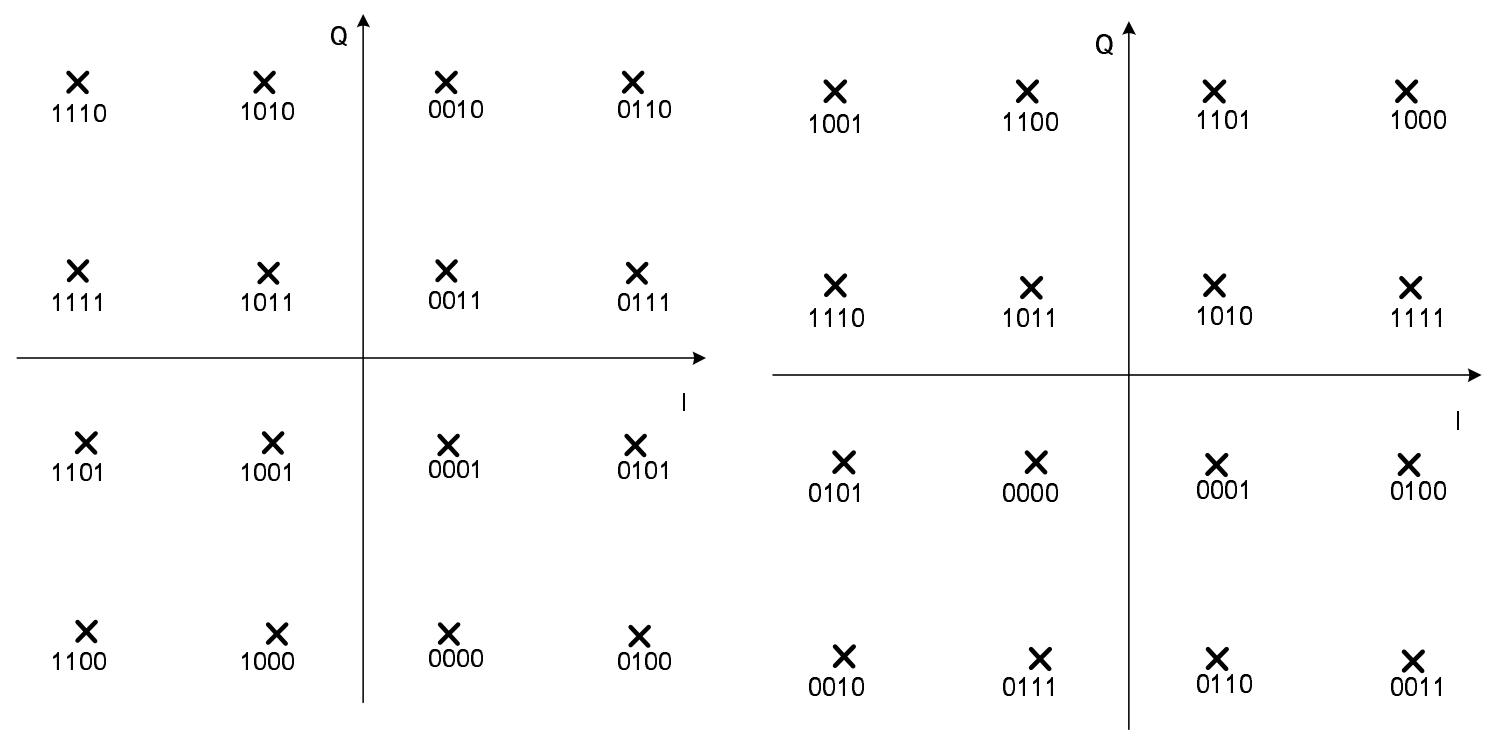

(a)

(b)

Figure 2.4: 16QAM signal set with (a) gray-labeling (b) set-partioning labeling

where $w(\mathbf{v})=\sum_{j=0}^{n-1} v_{j}$ is the Hamming Weight which represents the number of ones in a binary vector, $\mathbf{v}$. The importance of gray labeling arises from the fact that in the case of erroneously decoding a received symbol, the probability that the decoder chooses a bordering symbol instead of the transmitted one is very high. Thus, with gray coding that would lead to only one bit being in error instead of the case of casually assigning sequential binary numbers to the symbols in the signal constellation.

Mapping of coded bits by set-partitioning, or multilevel/phase channel signals [19], maximizes the Euclidean distance between signals with similar mapping (i.e. whose labellings have a Hamming distance of one). Coding gains achieved by this mapping are meant for state-oriented trellis coding rather than block coding. This mapping is a binary labeling obtained by an $m$-level binary tree partition of the signal set $\mathcal{S}$, where at each subsequent partition level or sub-constellation there exists a nondecreasing minimum Euclidean distance. The partitioning for a constellation with $M$ symbols is performed according to the following equation where the size of the $i^{t h}$ subconstellation $\mathcal{S}_{i}$ :

$$
\left|\mathcal{S}_{i}\right|=\frac{M}{2^{i}}
$$

where $i=m-1, \ldots, 2,1$ and $m=\log _{2} M$ (e.g. for a 16-QAM constellation the partitions 


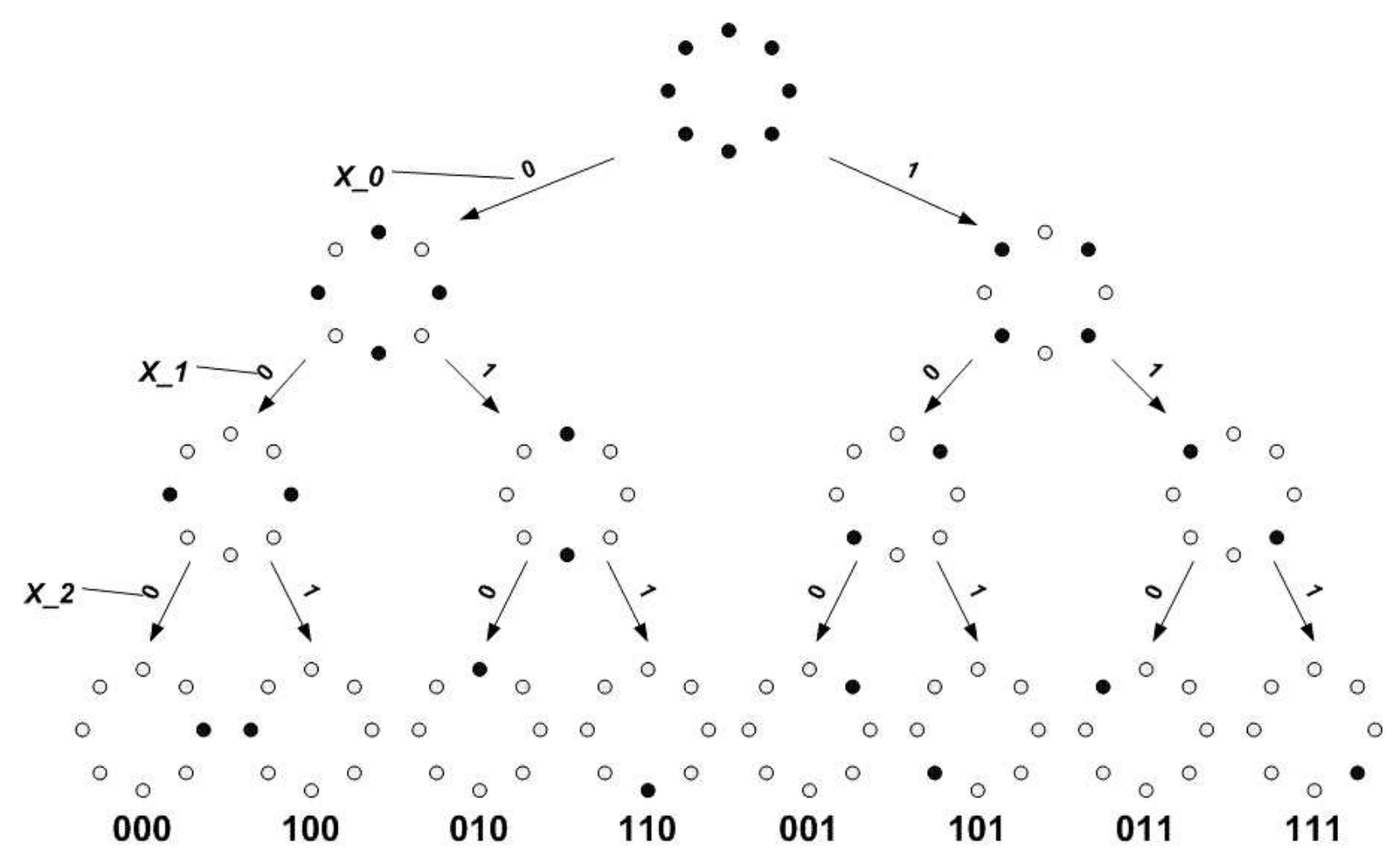

Figure 2.5: 8-PSK Set Partitioning

would be $M / 8, M / 4, M / 2, M / 1$ or $2,4,8,16)$. An example of set-partitioning mapping of 8-PSK can be seen in Fig. 2.5. In the figure the signals in the constellation whose labellings have 1 as the least significant bit is broken into one subconstellation and 0 into another. This process is performed on each subconstellation until we end up with $M$ subconstellations.

\subsubsection{Coded Modulation}

In the case where bandwidth efficiency is essential to the design of the system, a more effective system design can be obtained by using Coded Modulation (CM) (i.e. combining both modulation and coding) [6]. A CM scheme can be viewed as the concatenation of an encoder with a modulator where coding is performed onto an expanded modulation signal set (relative to that needed for uncoded transmission). Interleaving at the symbol level can be introduced before the modulation in order to overcome burst errors. CM finds applications on bandlimited channels such as voiceband telephone, terrestrial microwave, satellite and mobile channels. 


\subsubsection{BICM}

In modern wireless systems, bit interleaving is preferred over symbol interleaving because it offers improved diversity when communicating over Rayleigh channels [10]. The resulting interleaving is called bit interleaved coded modulation (BICM) and includes binary channel coding, bit interleaving, and bit-to-symbol mapping stages that are performed separately. Since BICM separates channel coding from modulation, it does not maximize Euclidean distance but instead results in a Hamming distance that is higher than that for Trellis Coded Modulation [20]. Assuming an infinite depth of the bit-interleaving, BICM transforms the multilevel signal constellation into $m$ parallel and independent binary channels, where each channel would carry one binary digit from the symbols in the constellation. Therefore, when calculating the log likelihood ratios at the receiver side, the equations (2.12) and (2.13), to be given in section 2.3, are used to transform any symbol metrics to bit metrics. Similarly, BICM capacity calculations are referenced to parallel independent channels, hence adding the capacities of the channels. BICM achieves large spectral efficiencies by combining a low rate binary channel code with a higher order signal constellation. As shown in [10], the performance of a BICM-constrained system can differ significantly from that of a system with an unconstrained Gaussian input, especially at high spectral efficiency.

\subsection{System Model}

The system model is as shown in Fig. 2.6. The system uses BICM [10], and hybrid automatic repeat request (hybrid-ARQ) [3], which will be described in section 4.2. The transmitter passes a length $K$ binary message $\mathbf{u}$ into a binary encoder, producing a codeword $\mathbf{c}^{\prime}$ of length $N$ bits. The codeword is bitwise interleaved, producing the vector $\mathbf{c}$, which is passed into an $M$-ary modulator. The modulator produces a length $\left\lceil N / \log _{2} M\right\rceil$ vector $\mathbf{x}$ of complex M-ary symbols drawn from the signal set $\mathcal{S}$. The modulated codeword is broken into $B_{\max }$ equal-length blocks, denoted $\mathbf{x}[b], 1 \leq b \leq B_{\max }$. The length of each block is $L=\left\lceil N /\left(B_{\max } \log _{2} M\right)\right\rceil$ symbols and the rate of each block is $R=K / L$.

The $b^{\text {th }}$ block is transmitted with average energy per symbol $\mathcal{E}_{s}=E\left\{|\mathbf{x}|^{2}\right\}$ over a block 


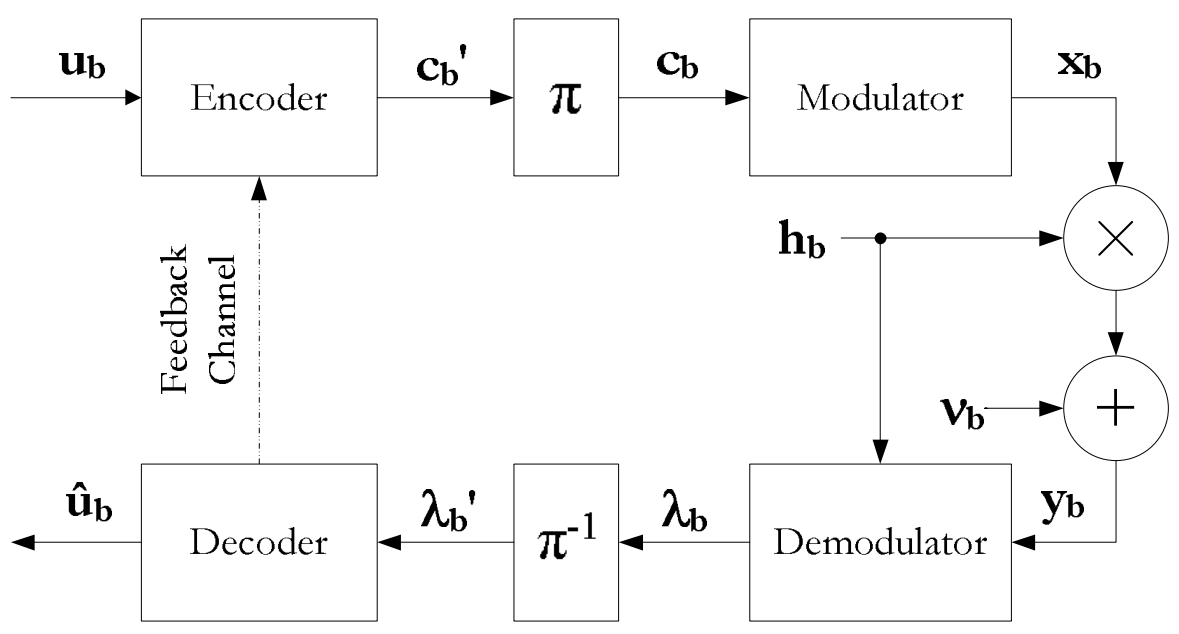

Figure 2.6: System model. $\pi$ denotes interleaving at the bit level.

fading channel so that the received signal is:

$$
\mathbf{y}[b]=h[b] \mathbf{x}[b]+\nu,
$$

where $\boldsymbol{\nu}$ is a vector of complex Gaussian noise whose dimensions match $\mathbf{x}[b]$ and whose components are zero-mean i.i.d. circularly symmetric Gaussian with variance $N_{o} / 2$ in each complex direction, and $h[b]$ is a complex scalar channel gain assumed to be independent from block to block and constant for the duration of each block. Without loss of generality, $E\left\{|h[b]|^{2}\right\}=1$ so that the average received energy per symbol is the same as the transmitted symbol energy.

Each received symbol in $\mathbf{y}[b]$ is passed through a demodulator that produces log-likelihood ratio estimates of each of the $\log _{2} M$ bits associated with the symbol. Since demodulation is on a symbol-by-symbol basis, consider the demodulation process for a single symbol $y$. For each possible signal $x_{m}$ in the signal set $\mathcal{S}, 1 \leq m \leq M$, a log-likelihood is formed ${ }^{2}$ :

$$
\begin{aligned}
\Lambda_{m} & =\log p\left(x_{m} \mid y\right) \\
& =\log \frac{p\left(x_{m} \mid y\right)}{\sum_{x \in \mathcal{S}} p(x \mid y)},
\end{aligned}
$$

Where $p(x)$ is the pdf of $x$. Letting the likelihood $f(x \mid y)=\kappa p(x \mid y)$ for any arbitrary constant $\kappa$ that is common for all postulated symbols, and applying Bayes' rule, then (2.8) can be

\footnotetext{
${ }^{2}$ unless otherwise noted, log means natural logarithm.
} 
more conveniently rewritten as

$$
\begin{aligned}
\Lambda_{m} & =\log \frac{f\left(y \mid x_{m}\right)}{\sum_{x \in \mathcal{S}} f(y \mid x)} \\
& =\log f\left(y \mid x_{m}\right)-\log \sum_{x \in \mathcal{S}} f(y \mid x) \\
& \left.=\log f\left(y \mid x_{m}\right)-\max _{x \in \mathcal{S}} * \log f(y \mid x)\right],
\end{aligned}
$$

where the max-star operator is as defined in [21], and further discussed in section 5.1.3:

$$
\max _{i} *\left\{x_{i}\right\}=\log \left\{\sum_{i} e^{x_{i}}\right\} .
$$

Coherent detection is implemented by using:

$$
\log f(y \mid x)=-\frac{\mathcal{E}_{s}}{N_{o}}|y-h x|^{2} .
$$

Notice in Fig. 2.6 that the receiver has perfect channel state information (CSI) but that the transmitter does not use any CSI.

The receiver transforms the set of $M$ log-likelihoods that are calculated for each received symbol into a set of $\log _{2} M$ bitwise log-likelihood ratios (LLRs), one for each code bit associated with the symbol. To calculate the LLR for the $i^{\text {th }}$ bit of received symbol $y$, first partition the symbol set $\mathcal{S}$ into two disjoint sets, $\mathcal{S}_{i}^{(0)}$, which is the set of symbols whose $i^{\text {th }}$ bit is a 0 , and $\mathcal{S}_{i}^{(1)}$, which is the set of symbols whose $i^{\text {th }}$ bit is a 1 . The LLR of the $i^{\text {th }}$ bit, $1 \leq i \leq \log _{2} M$, is then:

$$
\begin{aligned}
\lambda_{i} & =\log \frac{p\left(c_{i}=1 \mid y\right)}{p\left(c_{i}=0 \mid y\right)} \\
& =\log \frac{\sum_{x \in \mathcal{S}_{i}^{(1)}} p(x \mid y)}{\sum_{x \in \mathcal{S}_{i}^{(0)}} p(x \mid y)}
\end{aligned}
$$

When symbols are equally likely, this may be expressed as

$$
\lambda_{i}=\max _{x \in \mathcal{S}_{i}^{(1)}}[\log f(y \mid x)]-\max _{x \in \mathcal{S}_{i}^{(0)}}[\log f(y \mid x)] .
$$

After the $b^{\text {th }}$ block has been received, then the corresponding bit likelihoods for all blocks that have been received so far are passed into a decoder. 


\subsection{Conclusion}

An overview of wireless communication systems was presented in this chapter. Having done so, the block fading channel, which is the focus of this thesis, was fully described. Modulation, which is a physical layer technique that conveys information over the wireless channel by varying one or more of the parameters of the baseband signal, was then presented. Combining both coding and modulation, as in coded modulation, was put forward as a technique to provide bandwidth efficiency. The use of bit interleaved coded modulation was presented as an alternative for diversity gains in Rayleigh faded wireless channels. The chapter ended with an exposition of our system model for wireless links. 


\section{Chapter 3}

\section{The Information Theory of Block Fading Channels}

\subsection{Information Theory: Basic Definitions}

Information theory is a branch of mathematics founded by Claude Shannon in the 1940s, [22]. The theory addresses two main aspects of communication: the definition and measurement of information and the maximum rate that information can be sent through a communications channel (channel capacity). In this section, a review of the basics of Information Theory, taken from [22] and [23], are presented which lay the foundation for the analysis of the problem on hand.

\section{- Self Information}

Self information, which was defined by Shannon, is a measure of information content of a message $X$ :

$$
I(x)=-\log p[x]
$$

where $p[x]=\operatorname{Pr}(\mathcal{S}=X)$ is the probability of the message $X$ taken from the signal space $\mathcal{S}$ (i.e. the probability mass function) ${ }^{1}$.

\footnotetext{
${ }^{1}$ When the $\log$ is base 2 , the unit is in bits, whereas the unit is in nats when the log is base $e$.
} 


\section{- Entropy}

Entropy is a numerical measure of the uncertainty of an outcome. Entropy assigns a measure of uncertainty to the occurrence of an event which in our case is the uncertainty of a random variable.

The entropy $H_{X}$ of a discrete random variable $X$ is the expected value of the uncertainty of its outcomes

$$
H(X) \triangleq E_{x}[I(x)]=-\sum_{x \in \mathcal{X}} p[x] \log p[x]
$$

In the case of a continuous random variable, the sum can be replaced with an integral and densities can be used instead of probability mass functions. This is also called differential entropy:

$$
H(X) \triangleq-\int_{X} p(x) \log p(x) d x
$$

\section{- Mutual Information}

Mutual information measures how much information can be obtained about one random variable by observing another. Take two random variables, $X$ and $Y$; the mutual information is the amount of uncertainty removed from $X$ when $Y$ is known (i.e. thought of as the distance between $p[x, y]$ and $p[x] p[y]$, which is 0 when $X$ and $Y$ are independent). The mutual information $I(X, Y)$ as defined by [24] for discrete random variables is:

$$
I(X, Y)=\sum_{x, y} p[x, y] \log \frac{p[x, y]}{p[x] p[y]}
$$

where $p[x, y]$ is the conditional probability of $x$ given $y . x$ and $y$ range over all the values that $X$ and $Y$ can jointly take on.

As for continuous random variables, again by substituting the sum with an integral and densities instead of probability mass functions,

$$
I(X, Y)=\iint p(x, y) \log \frac{p(x, y)}{p(x) p(y)} d x d y
$$


Another way to express mutual information is:

$$
I(X, Y)=H(X)-H(X \mid Y)
$$

where $H(X \mid Y)$ is the conditional entropy defined as:

$$
H(X \mid Y)=E_{X, Y}(-\log p[x \mid y])
$$

\section{- Channel Capacity}

The capacity, $C$, of a channel with input $X$ and output $Y$ is found by maximizing the mutual information over all possible input distributions:

$$
C=\max _{p(x)} I(X, Y)
$$

Shannon's coding theorem and converse proved the existence of a code that could achieve a data rate close to capacity with negligible probability of error, and that any data rate higher than capacity could not be achieved without an error probability bounded away from zero (i.e. the highest rate at which information can be sent over the channel with arbitrarily low probability of error is the channel capacity).

\subsection{Ergodic Capacity}

The Shannon capacity of fading channels can be defined in a number of ways. The most straightforward interpretation is the ergodic capacity. A channel is classified as ergodic if the input signal is long enough to experience all the states of the channel [20]. This approach assumes that the coherence time $\left(T_{0}\right)$, the symbol time $\left(T_{s}\right)$ and the codeword transmission time $\left(T_{c}=N_{s} T_{s}\right.$ where $N_{s}$ is the number of symbols per codeword) are related by $T_{s}<T_{0}<<T_{c}$. This implies that the codeword spans a large number of coherence times.

\subsubsection{AWGN Capacity}

When there are no constraints on the input signal and the channel is an additive white Gaussian noise (AWGN) channel with complex-valued input $X$ and output $Y$, Fig. 3.1, (3.8) 


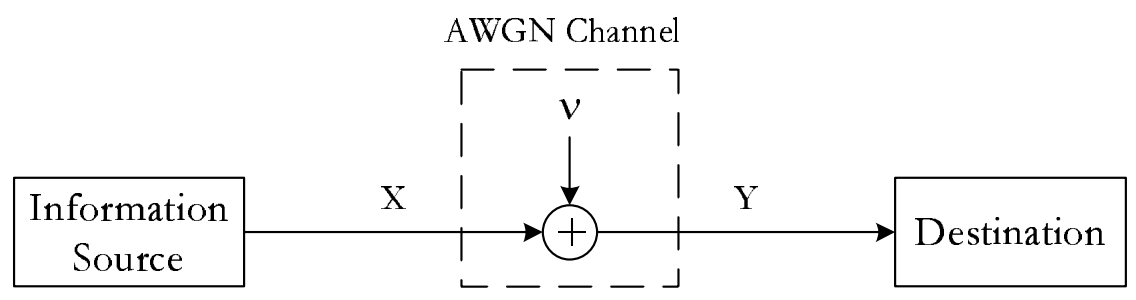

Figure 3.1: System model for input signal $X$ and output signal $Y$ in an AWGN channel with Complex Gaussian noise $\boldsymbol{\nu}$

is maximized by letting the input $p(x)$ take on a complex Gaussian distribution. This results in the classic unconstrained AWGN channel capacity²:

$$
C(\gamma)=\log (1+\gamma)
$$

where $\gamma=\mathcal{E}_{s} / N_{o}$ is the SNR and the capacity takes on units of bits per channel use (i.e. transmitted symbol).

Rather than using Gaussian distributed symbols, practical systems use symbols drawn from a finite signal set $\mathcal{S}$, usually with equal probability. Under such modulation constraints, $p(x)$ is a fixed function of $\mathcal{S}$, and since there is nothing to maximize over, the capacity is merely the mutual information given by (3.5) with $p(x)$ determined by the modulation constraint.

After some manipulation, (3.5) and (3.8), can be written in terms of the symbol likelihood $\Lambda_{m}$, equation (2.8), as the expectation [10]:

$$
\begin{aligned}
C(\gamma) & =E_{x_{m}, \nu}\left[\log M+\log p\left(x_{m} \mid y\right)\right] \\
& =\log M+E_{x_{m}, \nu}\left[\Lambda_{m}\right] \text { nats/symbol } \\
& =\log _{2} M+\frac{E_{x_{m}, \nu}\left[\Lambda_{m}\right]}{\log 2} \text { bits/symbol, }
\end{aligned}
$$

where the expectation is over all symbols $x_{m} \in \mathcal{S}$ and complex noise samples $\nu$ with SNR equal to $\gamma$. This expression represents the coded modulation (CM) capacity and can be evaluated either by numerical integration $[4,25]$ when the dimensionality is limited to 2dimensions, or Monte Carlo integration [10] for any dimensionality.

\footnotetext{
${ }^{2} C(\gamma)=\frac{1}{2} \log (1+\gamma)$ if input/output were real-valued
} 
If the system is further constrained to use BICM [10], then the channel is essentially transformed into $\log _{2} M$ parallel binary channels. The capacity of the $i^{\text {th }}$ binary channel, $1 \leq i \leq \log _{2} M$ is

$$
C_{i}(\gamma)=E_{c_{i}, \nu}\left[\log 2+\log p\left(c_{i} \mid y\right)\right] \text { nats/symbol }
$$

where the expectation is over the two possible code bits $c_{i} \in\{0,1\}$ and the complex noise samples $\nu$ with $\operatorname{SNR} \gamma$. After some manipulation, this can be expressed in terms of the binary LLR $\lambda_{i}$ as:

$$
\begin{aligned}
C_{i}(\gamma) & =\log (2)-E_{c_{i}, \nu}\left[\max *\left\{0,(-1)^{c_{i}} \lambda_{i}\right\}\right] \text { nats/symbol } \\
& =1-\frac{E_{c_{i}, \nu}\left[\max *\left\{0,(-1)^{c_{i}} \lambda_{i}\right\}\right]}{\log 2} \quad \text { bits/symbol. }
\end{aligned}
$$

Refer to section 5.1.3 for more information on the max* operator.

Since the capacities of parallel Gaussian channels add [24], the overall capacity of the BICM system is found by adding the capacities of the individual binary channels:

$$
C(\gamma)=\sum_{i=1}^{\log _{2} M} C_{i}(\gamma)
$$

As an example, the capacity when $\mathcal{S}$ is constrained to be either QPSK or 16-QAM is shown in Fig. 3.2. For comparison purposes, the unconstrained capacity (3.9) is also shown. For 16-QAM, both the CM and BICM capacities are shown. While the CM capacity does not depend on how bits are mapped to symbols, for BICM it does. The BICM-constrained capacity for two typical symbol mappings are shown, gray-labelling and set-partitioning (SP). While both BICM capacities are inferior to the CM capacity, the BICM capacity with gray-labelling is very close to the CM capacity, especially for $C(\gamma)>2$.

\subsubsection{Ergodic Rayleigh Fading Channel Capacity}

In contrast to the AWGN channel, the input signal $X$ is multiplied by a random channel gain $H$ in a Rayleigh fading channel. The input-output relationship of the system can then be described by the complex Gaussian random variable $H$ with zero mean such that $|H|$ has 


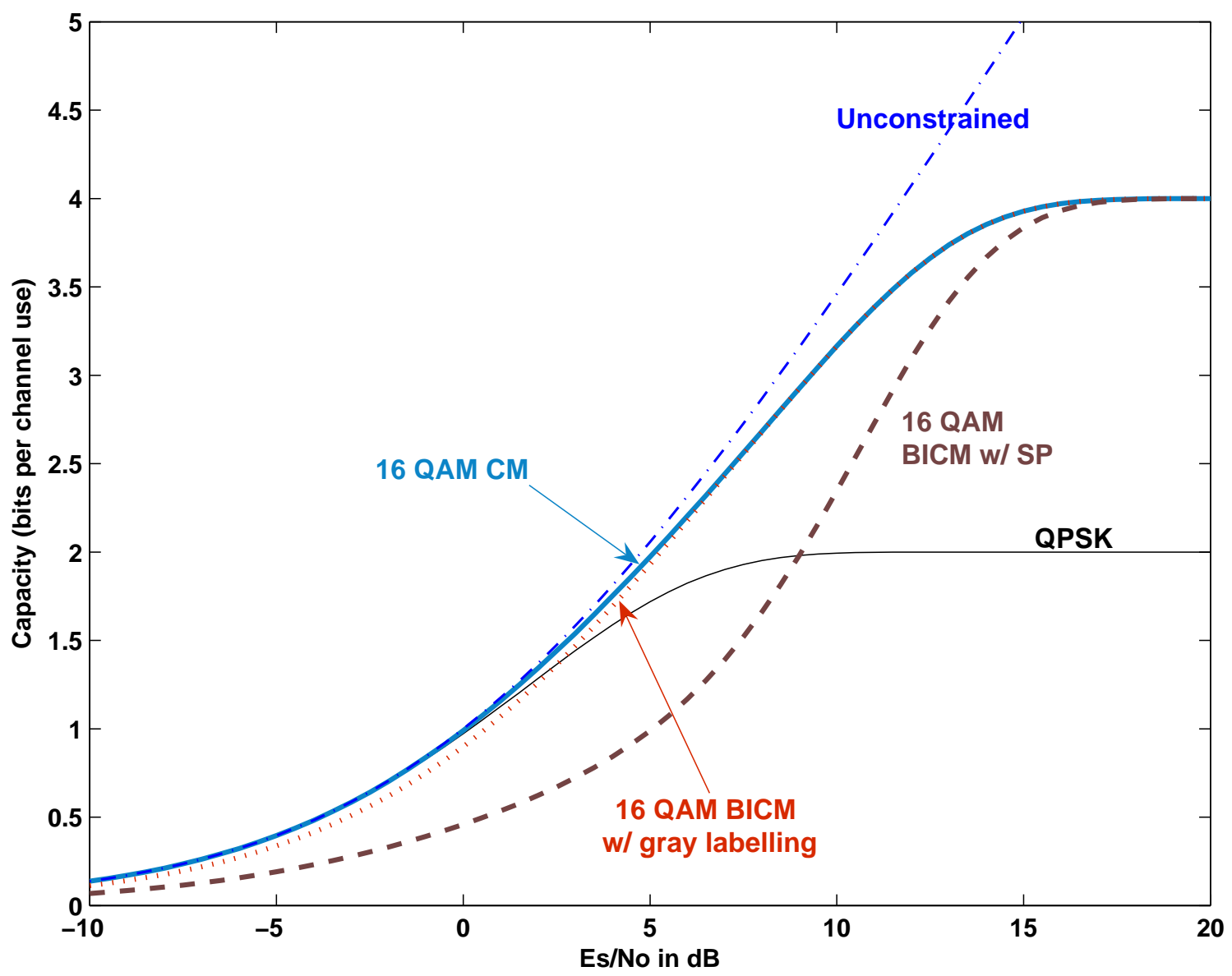

Figure 3.2: Capacity of QPSK, 16-QAM, and unconstrained (Gaussian-input) modulation in AWGN. For 16-QAM, the CM capacity is shown as is the BICM capacities for two types of symbol mappings (gray-labelling and set-partitioning (SP)).

a Rayleigh pdf, whose value affects the amplitude of the received signal. A representation of the system model with output signal $Y$ and Complex Gaussian noise $\boldsymbol{\nu}$ with Rayleigh fading is:

$$
Y=H X+\nu
$$

When the fading values are independently and identically distributed, then the values of $H$ form an ergodic process. The average or Shannon capacity with no constraints on the input signal in a Rayleigh faded channel is then maximized by letting the input $p(x)$ take on a Gaussian distribution. In an independent Rayleigh fading channel with channel state information at the receiver side only, the unconstrained ergodic Rayleigh channel capacity is $[20]$ : 


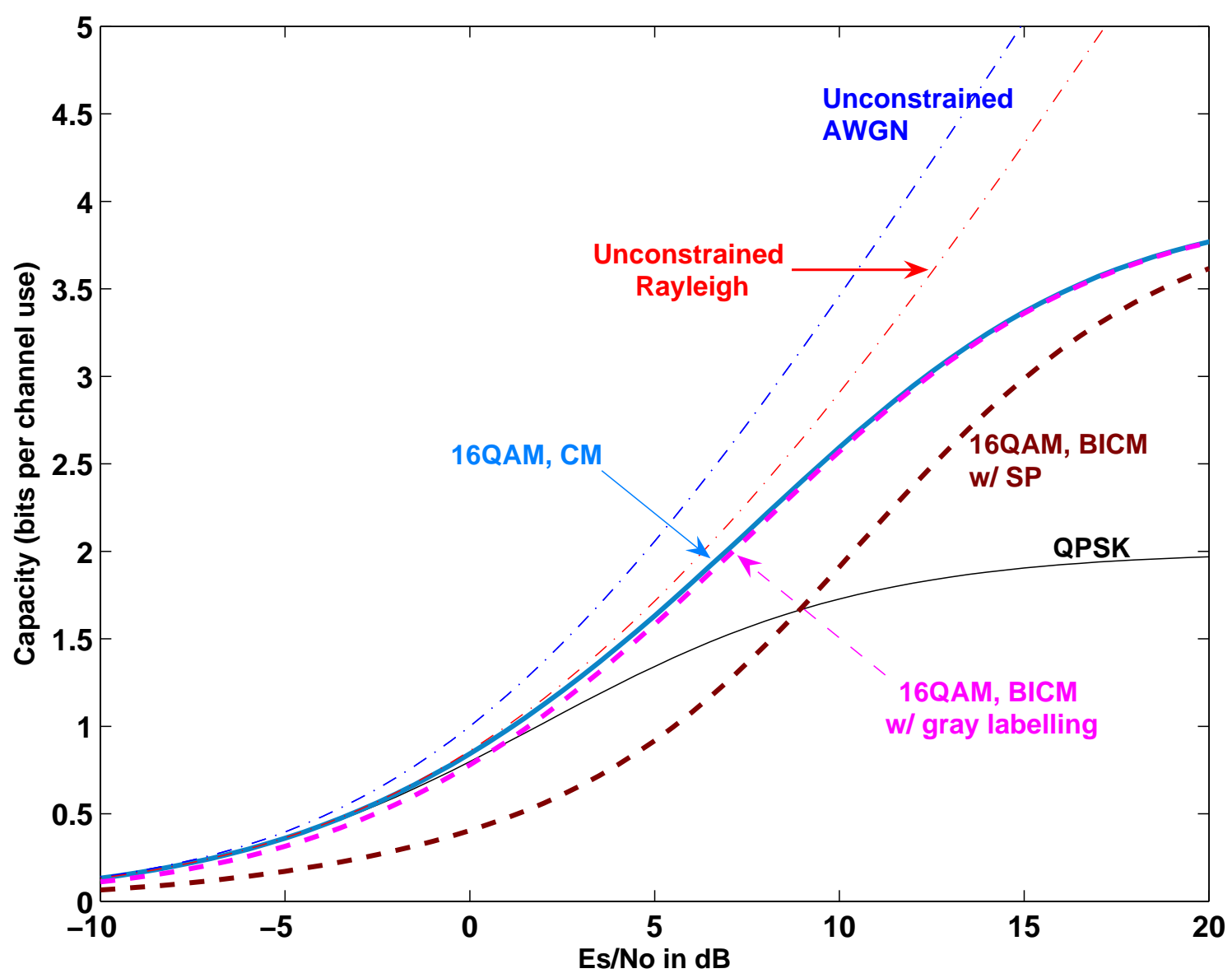

Figure 3.3: Capacity of QPSK, 16-QAM, and unconstrained (Gaussian-input) modulation in an ergodic Rayleigh fading channel. For 16-QAM, the CM capacity is shown as is the BICM capacities for two types of symbol mappings (gray-labelling and set-partitioning (SP)). The unconstrained modulation capacity curve for AWGN is also shown for comparison.

$$
\begin{aligned}
C(\gamma) & =E_{H}\left[\log \left(1+|H|^{2} \gamma\right)\right] \\
& =-\frac{1}{\ln 2} \exp \left(\frac{1}{\gamma}\right) E_{i}\left(-\frac{1}{\gamma}\right)
\end{aligned}
$$

where $E_{i}(x) \triangleq \int_{-\inf }^{x} \frac{e^{t}}{t} d t$ is the Cauchy principal value integral. In (3.15) one should observe that the channels are Gaussian with an attenuation factor of $|H|$ for every channel use, conditioned on the value of $H$. Furthermore, when $|H|$ is Rayleigh, $|H|^{2}$ is exponentially distributed.

Fig. 3.3 compares the unconstrained Gaussian input capacity in ergodic Rayleigh fading, as well as in AWGN channels. The QPSK and 16-QAM modulation constrained capacities 
are also shown. For 16-QAM, the CM capacity is shown as is the BICM capacities for two types of symbol mappings, gray-labelling and set-partitioning. The capacity of 16-QAM CM and 16-QAM BICM with gray labeling in Rayleigh fading for $\frac{E_{s}}{N_{o}}=10 \mathrm{~dB}$ is 2.5 bits per channel use, while for QPSK it is 1.73 bits per channel use. When compared to the capacity in an AWGN channel, Fig. 3.2, 16-QAM CM and 16-QAM BICM with gray labeling have a capacity of 3.25 bits per channel use and QPSK's capacity is 2 bits per channel use.

\subsection{Capacity Considerations in a Block Fading Chan- nel}

In block fading, the codeword is broken into $B$ blocks and each block is sent over an independent channel. Because the fading coefficient $h[b]$ of the $b^{t h}$ block is constant for the entire duration of the block, the channel during one block is conditionally Gaussian (conditioned on $h[b]$ ). However, since the fading coefficient is random, then so is the instantaneous SNR of the $b^{t h}$ block, which we denote $\gamma_{b} \equiv|h[b]|^{2} \mathcal{E}_{s} / N_{0}$, and therefore so is the corresponding capacity $C\left(\gamma_{b}\right)$. For Rayleigh block fading, $|h[b]|$ is Rayleigh and $|h[b]|^{2}$ is exponentially distributed.

The blocks could be encoded in such a way that all $B_{\text {max }}$ blocks are identical (a repetition code), in which case the blocks will be diversity-combined at the receiver by adding up the LLR's of each block. More generally, incremental redundancy could be used, whereby each block is obtained by puncturing a low rate mother code. With incremental redundancy, a different part of the codeword is transmitted each time, and after the $b^{\text {th }}$ block, a receiver will pass the rate $R_{b}=R / b$ code that it has until then received through its decoder (codecombining).

When code-combining is used, then the capacities of the $B$ blocks add, since each block is sent over an independent Gaussian channel. The resulting capacity is:

$$
C\left(\gamma_{1}, \ldots, \gamma_{B}\right)=\frac{1}{B}\left(\sum_{b=1}^{B} C\left(\gamma_{b}\right)\right),
$$

where the $\frac{1}{B}$ term is needed because blocks are orthogonal and therefore effectively occupy only $1 / B^{t h}$ of the channel. 
For diversity combining, the SNRs add and so the capacity when $B$ blocks are transmitted is:

$$
C\left(\gamma_{1}, \ldots, \gamma_{B}\right)=\frac{1}{B} C\left(\sum_{b=1}^{B} \gamma_{b}\right)
$$

When there are no modulation constraints, the capacities in (3.16) and (3.17) are found from the unconstrained AWGN capacity (3.9), while when there are modulation constraints equation (3.10) or equations (3.12) and (3.13) must be used for CM and BICM, respectively.

When $B$ is finite, the channel is not ergodic, and therefore a Shannon-sense channel capacity does not exist. For finite $B$, a more relevant performance metric is the information outage probability, defined in [4] and [5] as the probability that the instantaneous capacity $C\left(\gamma_{1}, \ldots, \gamma_{B}\right)$ is less than the per block rate $R_{B}=R / B$,

$$
p_{0}\left(\gamma_{1}, \ldots \gamma_{B}\right)=P\left[C\left(\gamma_{1}, \ldots, \gamma_{B}\right)<R_{B}\right]
$$

Whenever $C\left(\gamma_{1}, \ldots, \gamma_{B}\right)<R_{B}$, an information outage occurs, and reliable signaling is not possible. The information outage probability is an information theoretic bound on the frame error rate (FER) in block fading, and thus no system can have a FER that is better than the information outage probability. For convenience, we use the shorthand notation $p_{0}(B)=p_{0}\left(\gamma_{1}, \ldots \gamma_{B}\right)$ for the remainder of this thesis to denote the information outage probability of a B-block fading channel.

As seen in Fig. 3.4, using incremental redundancy and code combining always outperforms that with repetition coding and diversity combining. Therefore, due to the superiority of incremental redundancy and code combining, the remainder of the thesis will focus on code combining.

Monte Carlo simulation results for information outage probabilities are shown in the example shown in Fig. 3.5. For numerical integration results for the unconstrained input case refer to Appendix A. The information outage probability of code-combining is plotted against SNR in Rayleigh block fading for rate $R_{B}=2$ bits per symbol and $B=\{1,2,3,4,10\}$. For each value of $B$, two curves are shown, one for an unconstrained Gaussian input [obtained by substituting (3.9) into (3.16) with $R_{B}=2$ ], and the other for a BICM constrained input using gray-labelled 16-QAM [obtained by substituting (3.12) into (3.13) and (3.16)]. As 


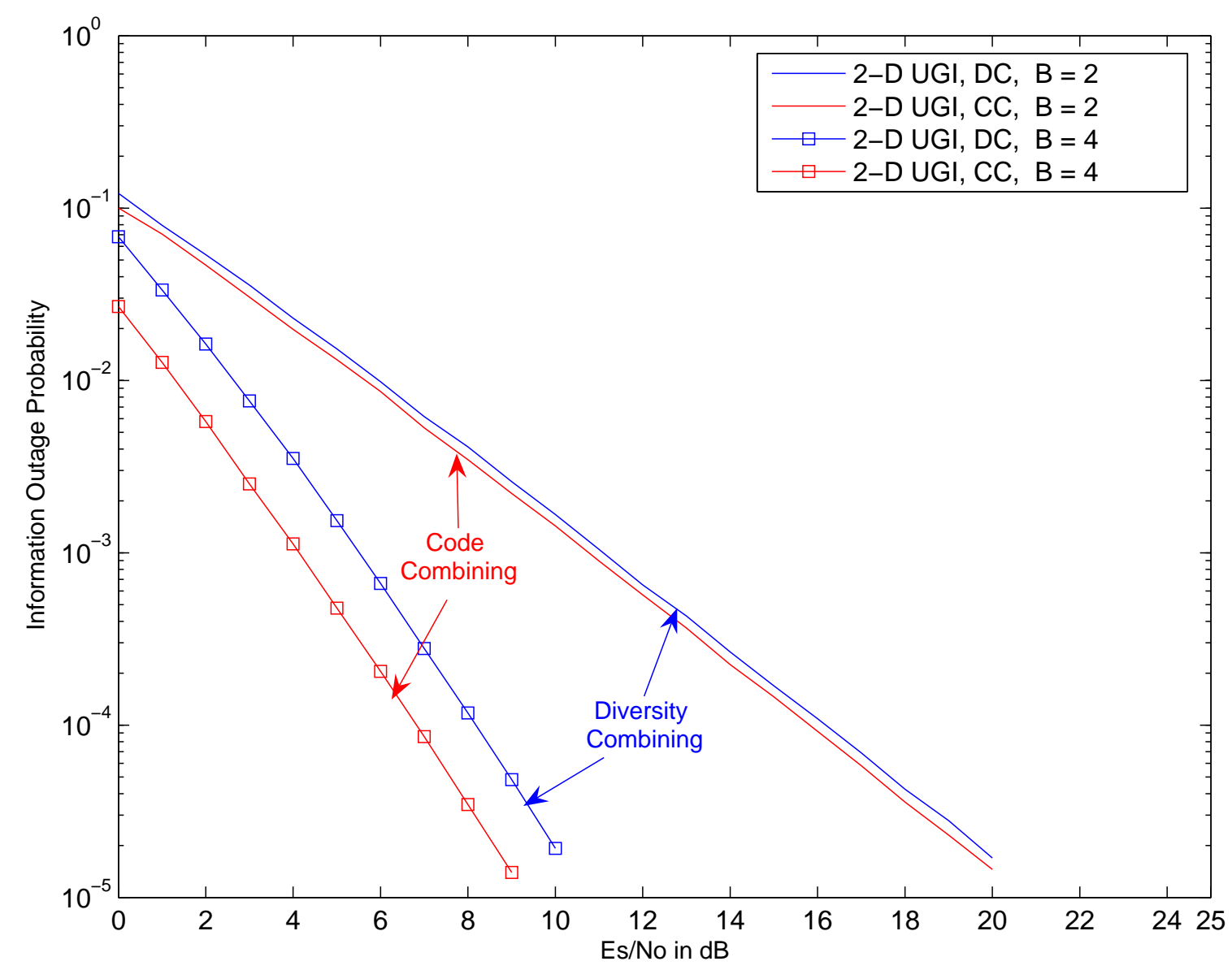

Figure 3.4: Information outage probability vs. SNR for unconstrained Gaussian input modulation in Rayleigh block fading with a per block rate $R_{B}=1 / 3$ and $B=2,4$ using both code and diversity combining.

expected, the information outage probability under modulation constraints is worse than without such constraints. However, the amount of the loss depends on the number of blocks per codeword. For $B=1$ the loss due to the modulation constraint is only about $0.5 \mathrm{~dB}$ for sufficiently high SNR, but for $B=2$ the loss is about $1.25 \mathrm{~dB}$. For $B=3$, the modulation constrained curve is less steep than that of the unconstrained curve, suggesting a loss of diversity. This is consistent with the observation made by Knopp and Humblet in [4] that "for practical (i.e. small) signaling alphabets, the attainable diversity order due to coding (which in Rayleigh fading corresponds to the slope of the information outage probability curve versus the signal-to-noise ratio (SNR) on a log-log scale) is generally smaller than $B$ ".

A more specific account for the discrepancy is discussed in [6]. Fabregas et al. explain 


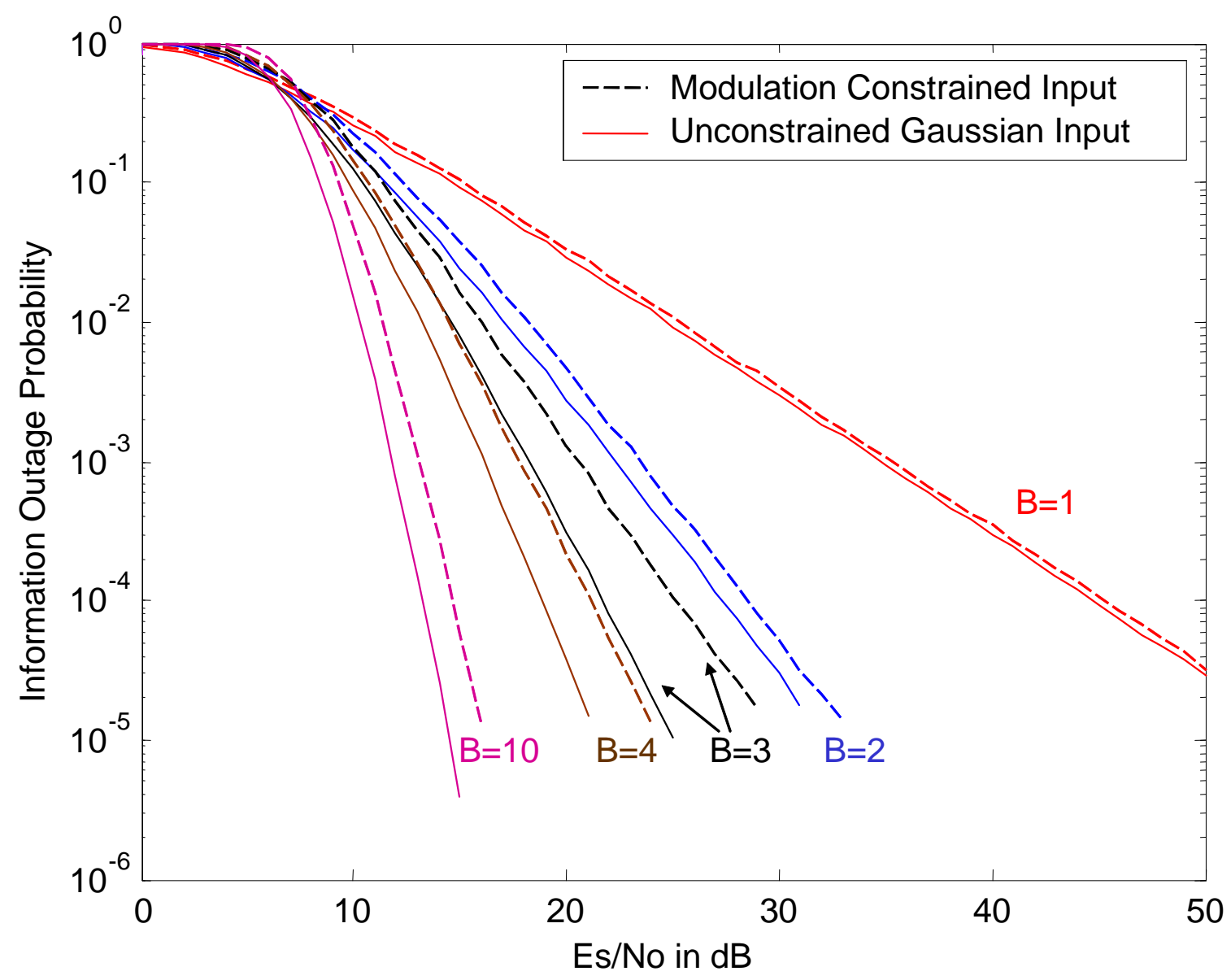

Figure 3.5: Information outage probability vs. SNR for unconstrained and gray-labelled 16QAM modulation in Rayleigh block fading with rate $R_{B}=2$.

that on this log-log scale, each curve becomes a straight line at high SNR. The slope of the line is $-d$, where $d$ is an integer in $[0, B]$ and is called the block diversity or SNR exponent. For an unconstrained Gaussian input channel, $d=B$, but under modulation constraints the diversity is upper-bounded by the Singleton bound

$$
d=1+\left\lfloor B\left(1-\frac{R_{B}}{\log _{2} M}\right)\right\rfloor .
$$

Since in this case $R_{B} / \log _{2} M=1 / 2, d=1,2,2,3$ and 6 for $B=1,2,3,4$ and 10, respectively. This behavior can be observed in the figure. For $B=1$ and 2, the outage probability under modulation constraints is worse than the unconstrained case, but asymptotically the two curves for the same value of $B$ have the same slope. However, for $B=3$ not only is the constrained case worse than the unconstrained case, but asymptotically it has the same slope 

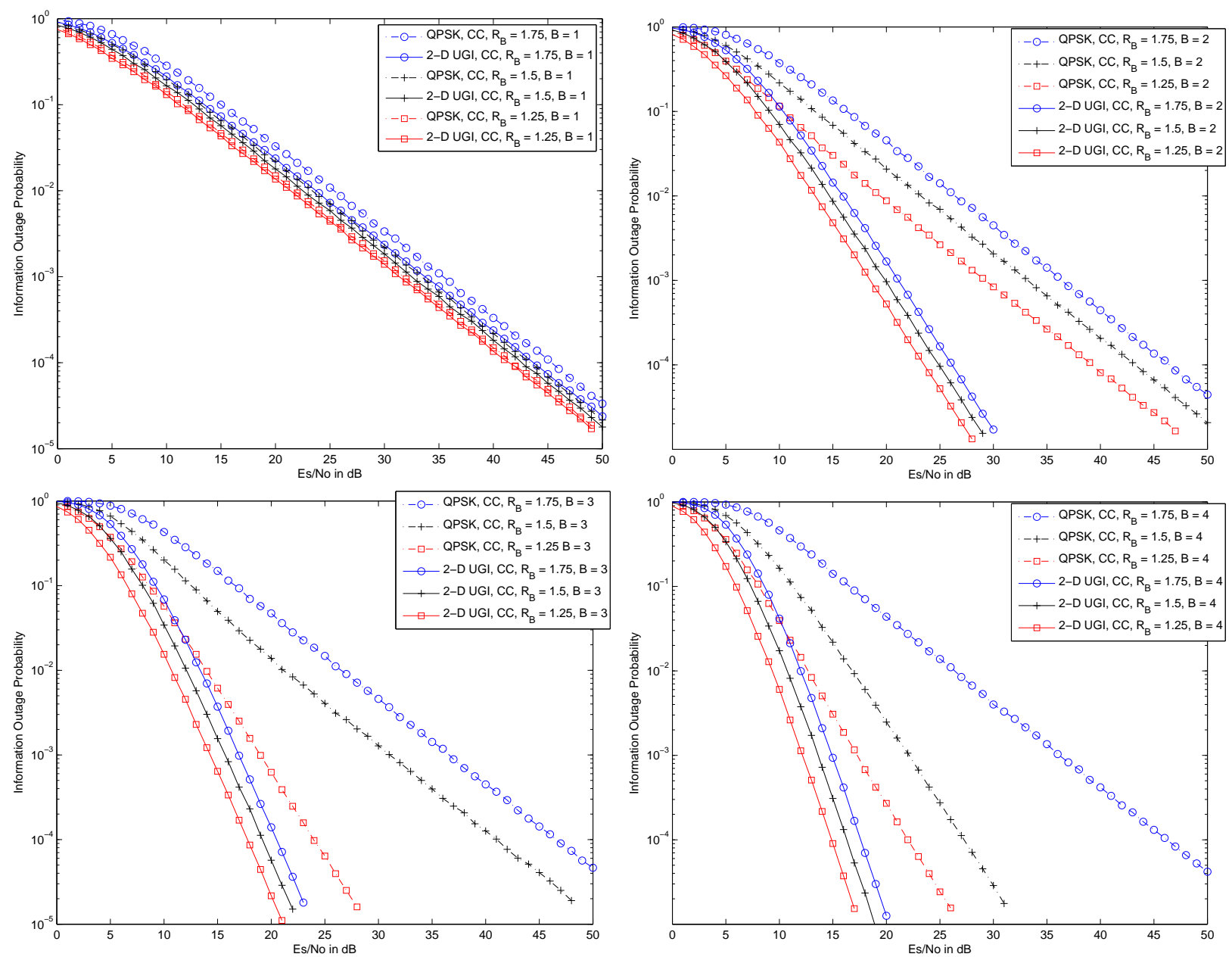

Figure 3.6: Information outage probability vs. SNR for unconstrained (solid line) and QPSK modulation (dashed line) in Rayleigh block fading for $B=1,2,3,4$ using code combining with different rates; $R_{B}=1.5,1.75$, and 2 .

as the $B=2$ unconstrained case. Similarly, the $B=4$ constrained case has the same slope as the $B=3$ unconstrained case. For $B=10$, the asymptotic slope for the constrained case is indeed 6 , though this is not obvious by looking at the figure because slope 6 and 10 look similar to the eye.

Information outage probability was further investigated for the unconstrained Gaussian input and QPSK constrained modulation for different rates. Fig. 3.6 provides plots for different block values $B=1,2,3,4$ with $R_{B}=1.25,1.5,1.75$. The behavior noticed is intuitive since the system experiences more outages as the rate increases. These results show that the system becomes more energy inefficient when operating at higher spectral 
efficiencies. This can be explained by looking at equation (3.19), where higher rates suffer higher diversity losses. This can be clearly observed for the case of $R_{B}=1.75$. The block diversity $d=1,1,1,1$ for $B=1,2,3,4$. The diversity losses for $R_{B}=1.25,1.5,1.75$ are shown as a function of different block values for the unconstrained Gaussian input, graylabeled 16QAM and QPSK modulation in Fig. 3.7. If the ratio of the rate to number of bits per symbol (i.e. $\frac{R_{B}}{\log _{2} M}$ ) is the same for both modulations, they both incur the same diversity loss. For example, if we use $R_{B}=2.5,3,3.5$ with 16QAM, we will end up with the same three rightmost curves as shown in Figs. 3.6/3.7.

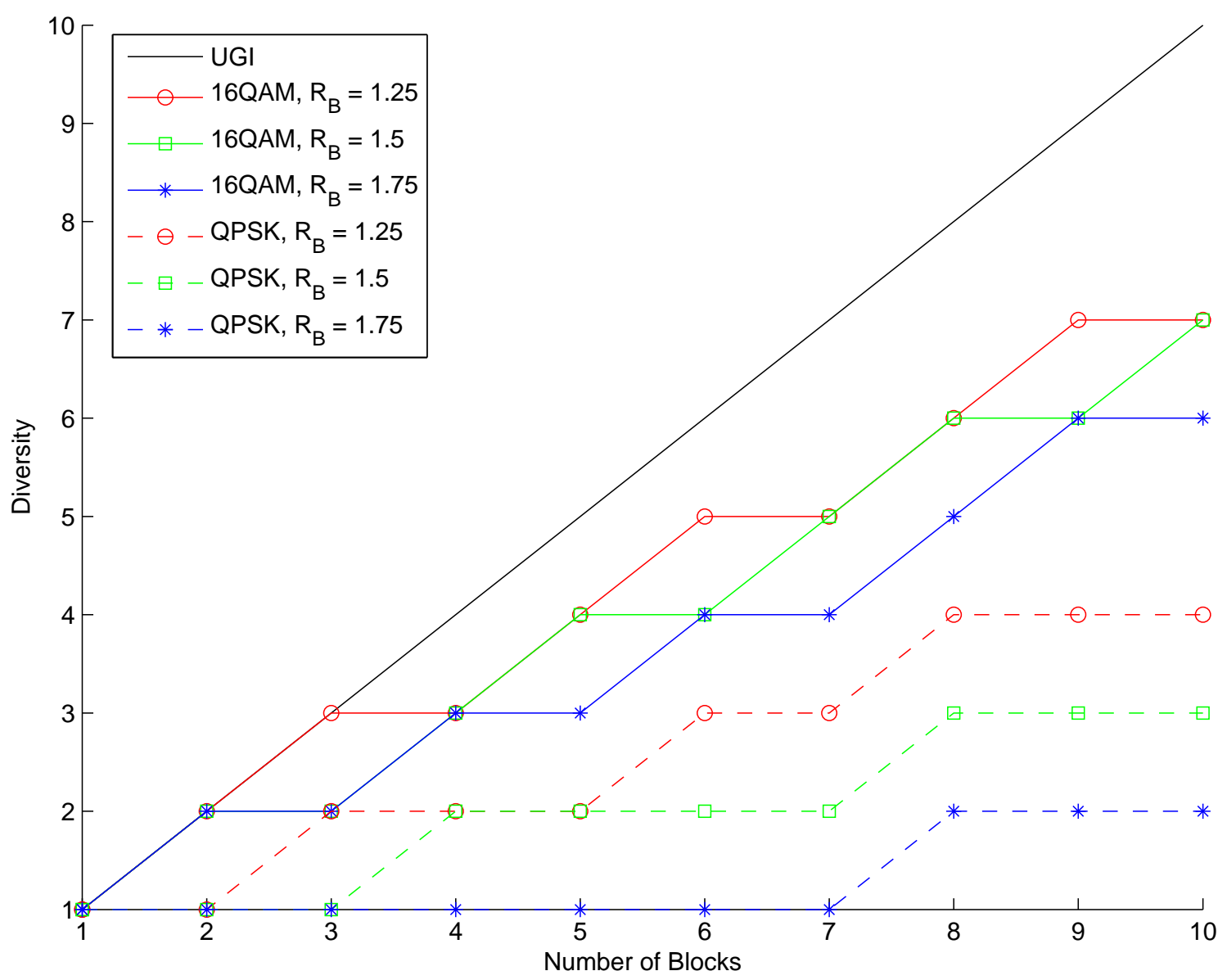

Figure 3.7: Diversity vs. number of block for the unconstrained Gaussian input (solid black), QPSK constrained modulation (dashed line), and 16 QAM constrained modulation(solid line) for different rates; $R_{B}=1.25$ (circle), 1.5(square), and 1.75(star). 


\subsection{Conclusion}

In this chapter, an overview of information theory has been presented in order to lay a foundation for the remainder of the thesis. The unconstrained AWGN and ergodic Rayleigh fading channel capacities were derived based on the fact that the input distribution was Gaussian thereby maximizing the mutual information over all possible input distributions. Block fading channels are parallel Gaussian channels, so their instantaneous capacity is the sum of the capacities of each block (at least for incremental redundancy and code combining). The influence of rate on the information outage probability and the diversity, which is related to the slope of the curve, was studied for both unconstrained Gaussian input channels, as well as for channels with constrained modulation. It was shown that the diversity of the system depends on the rate, number of blocks, and the choice of modulation.

Based on the information given in this chapter, chapter 4 will further study and provide an analysis of hybrid-ARQ in point-to-point communications for the block fading channel. Chapter 6 will provide the same analysis for hybrid-ARQ based relaying protocols under modulation Constraints. 


\section{Chapter 4}

\section{Hybrid Automatic Repeat Request}

\subsection{Introduction}

Automatic Repeat Request (ARQ) is a technique where a message is encoded by a low rate mother code and then partitioned into several blocks. Blocks are sent one by one until enough information is accumulated at the destination to correctly decode the message. ARQ thereby ensures that a data stream is delivered accurately to the end user despite errors that might occur during transmission. ARQ ensures a high level of reliability through the repeated retransmission of an erroneous frame. The source retransmits if one of the following two events occur:

1. the source receives a negative aknowledgement (NACK).

2. the source does not receive a valid acknowledgement (ACK) from the destination within a specific time duration.

Usually, when the error persists beyond a predetermined number of retransmissions, the source will move on to transmitting the next message in the queue.

There are several ways to implement ARQ namely, Stop-and-wait ARQ, Go-Back-N ARQ and Selective Repeat ARQ. The simplest implementation and the most bandwidth inefficient is Stop-and-wait ARQ. In Stop-and-wait ARQ the source transmits a single frame and then must await an ACK without sending any further frames. In this protocol, two sorts of error could occur. First, the frame that arrives at the destination could be damaged. The 
second sort of error is a damaged acknowledgment. Both these errors are remedied by a time-out parameter at the source, whereby if a certain time passes without receiving an ACK the source re-sends the frame again. Stop-and-wait is inefficient only if the source and the destination are far apart. Bluetooth [26], which is a wireless protocol for personal area networks (PANs), has quite acceptable performance since it uses Stop-and-wait over short distances.

\subsection{Hybrid-ARQ}

Hybrid-ARQ is a variation of the ARQ error control method. A hybrid ARQ algorithm consists of the combination of a Forward Error Correction (FEC) code, error detection and a retransmission scheme. In theory, this can be done using one code to do both error correction and detection. However, in practice, prior to transmission, the data block is encoded first with an error-detection code (e.g. Cyclic Redundancy Check or CRC) and then with an error-correction code such as a Reed-Solomon code or turbo code. Deployment of hybridARQ over wireless channels outperforms ordinary ARQ, though at the cost of increased implementation complexity (e.g. physical layer buffering, increased overhead). Due to their intrinsic capability to adapt to different channel conditions, hybrid-ARQ schemes are of great interest for transmission systems that operate over time-varying channels. Performance comparisons for various schemes of hybrid-ARQ in different radio channels can be found in the literature (e.g. [27] and references therein). A proper combination of FEC and ARQ provides higher reliability than an FEC scheme alone, and higher throughput than an ARQ scheme alone.

There are several hybrid-ARQ algorithms which can be differentiated according to the content of subsequent retransmissions. The simplest version of hybrid-ARQ is Type I hybridARQ. After receiving the block, the receiver decodes the message and checks for errors. Type I hybrid-ARQ does not buffer corrupted transmissions for later decoding, which makes it relatively simple to implement. Instead, each retransmitted packet is decoded without using the previously received packet(s).

Further improvements on the reliability performance of Type I hybrid-ARQ can be 
achieved by buffering the incorrectly received message instead of discarding it, which is the idea behind Type II hybrid-ARQ. If the same message (i.e. same coded data bits - repetition coding) is sent again, the information from both received messages can be combined (diversity/Chase combining [28]) before trying to decode the actual data. Re/transmissions can be sent using another scheme where additional parity bits are sent in the form of incremental redundancy. The receiver code combines the multiple received packets by forming a single low rate codeword. This combining of the information across various retransmissions results in a higher probability of successful decoding. This thesis focuses on incremental type II algorithms with code combining.

Another variation of hybrid-ARQ is Type III hybrid-ARQ. The difference between type III hybrid-ARQ and type II hybrid-ARQ is that the re/transmitted packets in Type III hybrid-ARQ can be decoded by themselves due to repeated information of user data bits in each re/transmission. Hence, Type III schemes are less efficient than Type II schemes. However, Type III has the benefit that each packet is self-decodable.

In incremental redundancy hybrid-ARQ, a message is encoded by a low rate mother code and then partitioned into several blocks. Blocks are sent one by one until enough information is accumulated at the destination to correctly decode the message. If the message is correctly decoded, an acknowledgement is sent back through a feedback channel (we assume here that the feedback channel is error and delay-free and that an ideal error detecting code allows the receiver to discriminate between correctly and incorrectly decoded messages). If the transmitter receives an acknowledgement, it will move on to the next message; otherwise, it will send the next block from the current message. This process continues until either the message is received correctly or the last $\left(B_{\max }\right)$ block is transmitted. In this scheme, effective coding is performed whereby (re)transmissions are coded differently rather than simply repeating the same coded bits as in diversity combining. That is, an alternative set of parity bits are sent in each retransmission. By employing combining techniques after every retransmission, a richer set of parity bits is available at the receiver, thereby improving the probability of reliable decoding. An example of incremental redundancy hybrid-ARQ is HSDPA where the data is first coded with a punctured $1 / 3$ Turbo code. HSDPA will be further examined in section 5.2. It is worth mentioning that all types of hybrid-ARQ can be 


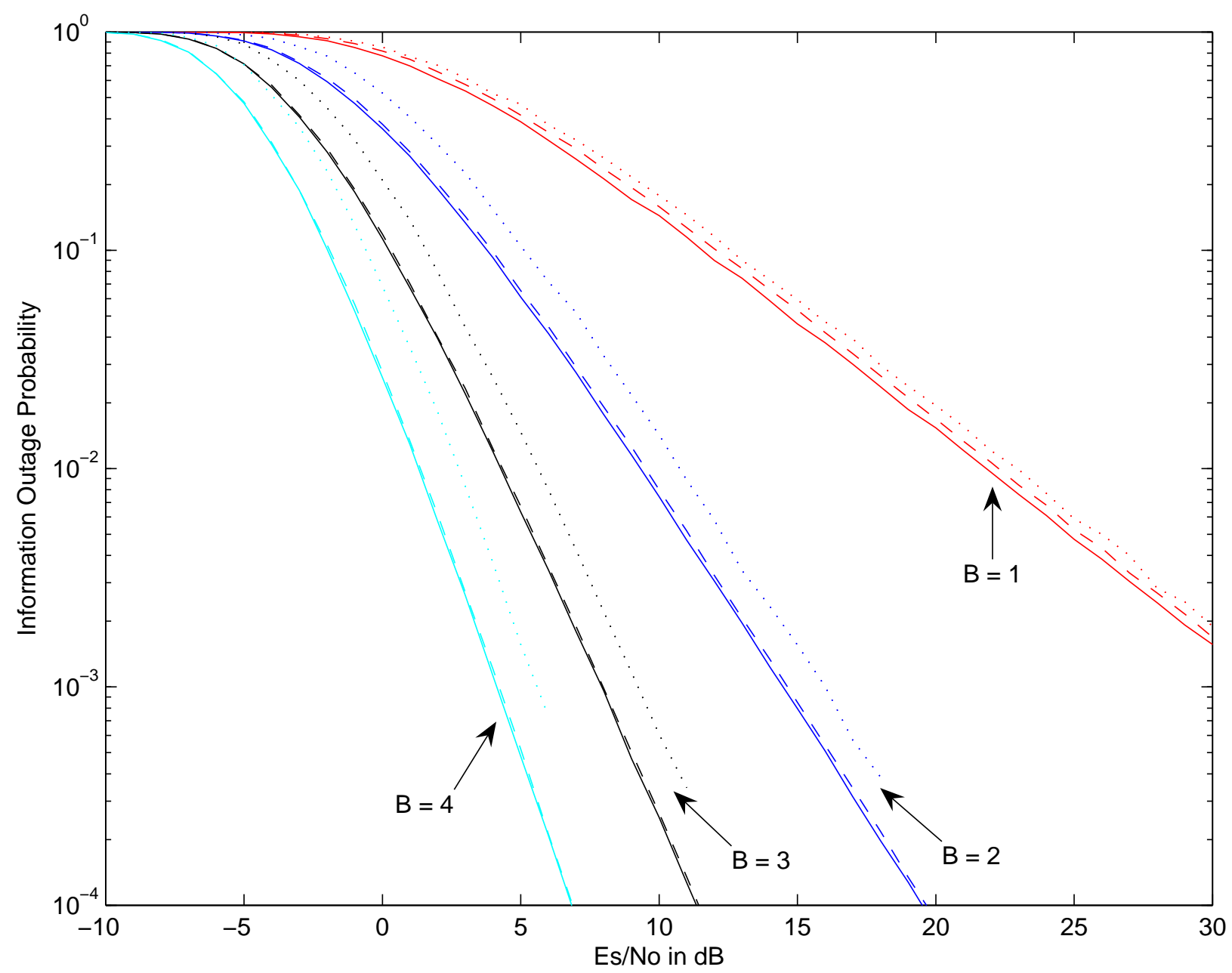

Figure 4.1: Information outage probability vs SNR in Rayleigh block fading using QPSK modulation with rate $R=3202 / 2400$. Unconstrained (solid line) and modulationconstrained (dashed line) theoretical limits are compared against the simulated performance of the HSDPA system (dotted line).

used with any of the ARQ protocol versions mentioned previously.

Often, the channel is uncorrelated from one block to the next, in which case a block fading model may be assumed. When using hybrid-ARQ in a block fading channel with modulation constraints, the code rate after the $B^{\text {th }}$ block has been transmitted is $R_{B}=R / B$. The upper-bound on diversity which was given by (3.19) is then transformed to:

$$
d=1+\left\lfloor B-\frac{R}{\log _{2} M}\right\rfloor \text {. }
$$

This implies that as long as $R<\log _{2} M$ (which must be true in practical systems) then $d$ is upper bounded by $B$ and there is no loss in diversity in hybrid-ARQ systems due to 
using modulation constraints. This can be clearly seen in Fig. 4.1 when compared to Fig. 3.5 in chapter 3. Fig. 4.1 shows that the slope (i.e. diversity) of the modulation constrained and HSDPA system suffers no loss, where the block diversity $d=1,2,3,4$ for the respective block values $B=1,2,3,4$.

\subsection{Throughput}

A key performance metric for hybrid-ARQ is its throughput, which is the number of correct bits conveyed per unit time. In [3], Caire and Tuninetti derived information-theoretic bounds on the throughput of hybrid-ARQ in block fading. The results built upon related work on the performance of standard block fading channels $[4,5]$, i.e. channels with a fixed rate and number of blocks per codeword, as described in section 3.3. The results in [3] placed no constraints upon modulation, and as a consequence, the input to the channel was assumed to be Gaussian distributed. However, practical systems do not use Gaussiandistributed modulation, and the computation of information-theoretic limits on the throughput of hybrid-ARQ with practical modulation constraints has until now remained an open problem.

Let the random variable $B$ indicate the number of hybrid-ARQ transmissions until the packet is successfully received. Initially, consider the case that there is no limit on the number of transmissions. The probability mass function (pmf) of $B$ is then given by:

$$
p_{B}[b]=\left(1-p_{0}(b)\right) \prod_{i=1}^{b-1} p_{0}(i) \text { for } b \geq 1
$$

where $p_{0}(b)$ is the information outage probability after the $b^{t h}$ attempt, as given by (3.18). An interpretation of (4.2) is that for $B=b$, the first $b-1$ attempts must fail while the $b^{\text {th }}$ attempt must succeed.

Often, an upper limit $B_{\max }$ is placed on the number of hybrid-ARQ transmissions. If the message is not received after $B_{\max }$ blocks have been transmitted, then an error is logged, and the system moves on to the next message. The pmf of $B$ with constraint $B_{\max }$ on the 
number of transmissions is

$$
p_{B}[b]= \begin{cases}\xi\left(1-p_{0}(b)\right) \prod_{i=1}^{b-1} p_{0}(i) & \text { for } 1 \leq b \leq B_{\max } \\ 0 & \text { otherwise }\end{cases}
$$

where $\xi$ is a normalization factor required to make $p_{B}[b]$ a valid pmf:

$$
\xi=\left[\left(1-p_{0}(b)\right) \prod_{i=1}^{B_{\max }-1} p_{0}(i)\right]^{-1}
$$

Let $\tau$ be the time between the start of consecutive blocks (which includes the time to transmit the block, process it, send an acknowledgement, and process the acknowledgement). Then the throughput, in bits per second, is:

$$
\eta=\frac{K}{\tau E[B]}
$$

where $E[B]$ is the expected value of $B$, and $K$ is the number of information bits per message. A more meaningful metric is the throughput efficiency, which is the ratio of correct bits to transmitted bits:

$$
\eta_{e f f}=\frac{1-p_{0}\left(B_{\max }\right)}{E[B]}
$$

Another metric of interest is the latency, which is the time between correctly decoded messages, and is given by $\tau / \eta_{\text {eff }}$ seconds.

\subsection{Numerical Results}

Numerical results for the throughput of hybrid-ARQ were produced by using Monte Carlo simulations. Parameters in the simulations were chosen in order to gain some insight on the loss due to the code used by the actual HSDPA system, which will be discussed in the next chapter. The number of information bits is $K=3202$ for QPSK and $K=4664$ for 16QAM. After rate matching, the block size is $L=2400$ QPSK symbols or $L=1920$ 16-QAM symbols. The maximum number of hybrid-ARQ transmissions per message is $B_{\max }=4$ where incremental redundancy with code-combining was used. 


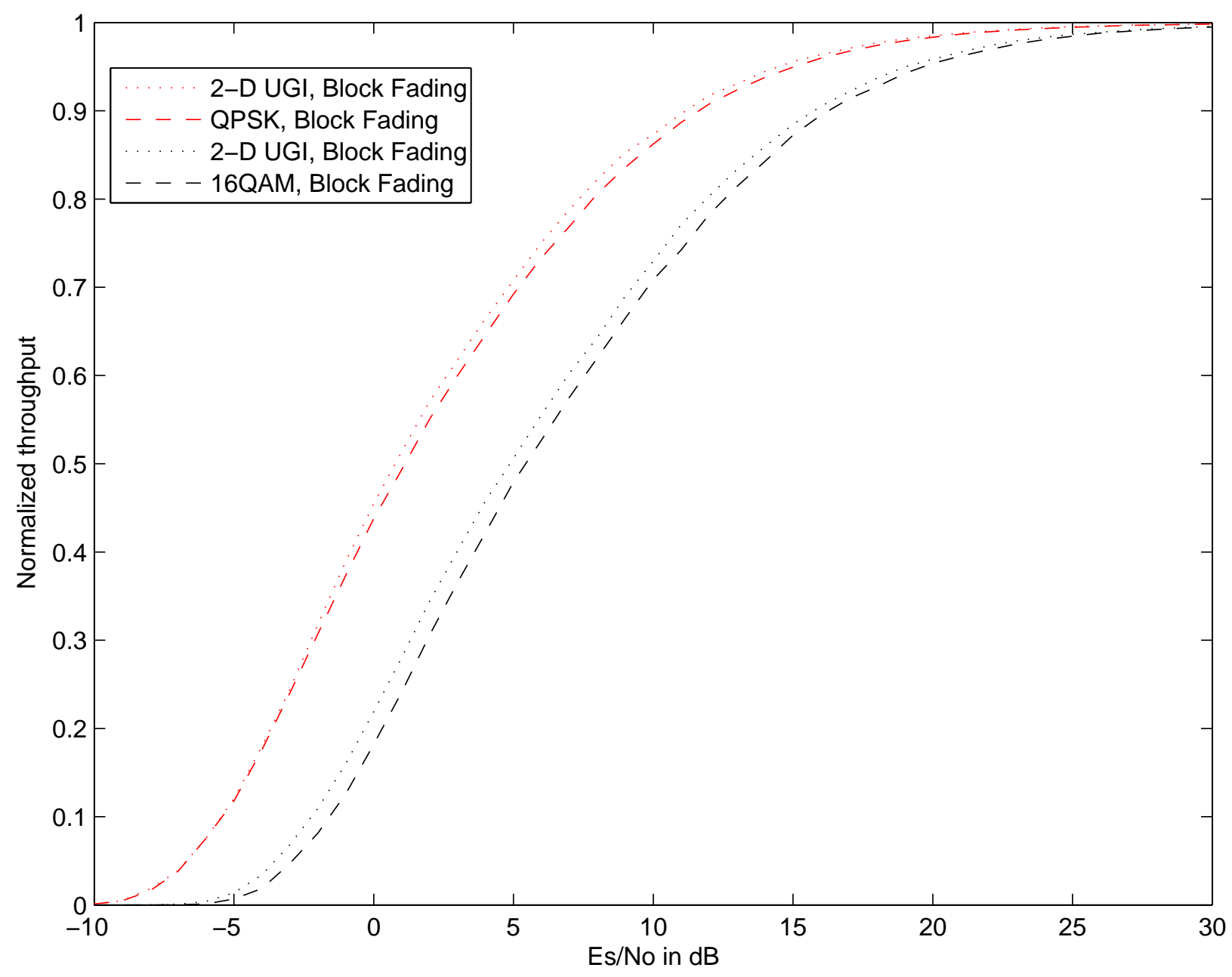

Figure 4.2: Throughput efficiency of hybrid-ARQ with code combining in Rayleigh block fading using QPSK or 16-QAM modulation. For each modulation type, the unconstrained and modulation-constrained theoretical limits are shown.

Fig. 4.2 shows throughput efficiency versus SNR in Rayleigh block fading using both QPSK and 16-QAM modulation. The figure shows two groups of two curves. The group on the left is for QPSK and the group on the right is for 16-QAM. Note that QPSK has better throughput efficiency than 16-QAM in this application because it has a lower per-block code rate $(R=3202 / 2400$ for QPSK and 4664/1920 for QAM). For each modulation type, two curves are shown. The left curve is the information-theoretic limit on throughput with an unconstrained (i.e. Gaussian-distributed) input, while the right curve is the informationtheoretic limit with a modulation-constrained input.

The results shown in Fig. 4.2 indicate the loss in throughput due to modulation con- 
Table 4.1: Value of normalized throughput at 0.5 for different rates

\begin{tabular}{|c||c||c|}
\hline & UGI & QPSK Mod. \\
\hline$R=1.25$ & 0.41 & 0.64 \\
\hline$R=1.5$ & 1.53 & 1.97 \\
\hline$R=1.75$ & 2.52 & 3.39 \\
\hline
\end{tabular}

straints. For instance, with QPSK, a throughput efficiency $\eta_{\text {eff }}=0.5$ is achieved at $\mathcal{E}_{s} / N_{0}=0.77$, and $1.12 \mathrm{~dB}$ for the unconstrained, and modulation-constrained cases, respectively. This implies that the loss due to modulation constraints is 0.35 . Similarly, for 16-QAM, a throughput efficiency $\eta_{\text {eff }}=0.5$ is achieved at $\mathcal{E}_{s} / N_{o}=4.88$, and $5.44 \mathrm{~dB}$ for the unconstrained and modulation-constrained cases, respectively. This implies that the loss due to modulation constraints is $0.56 \mathrm{~dB}$. It is interesting to note that for QPSK, the loss due to the modulation constraint diminishes at low throughput efficiency (e.g. $\eta_{e f f}<0.2$ ), while for QAM it does not (except at extremely small $\eta_{\text {eff }}$ ). These results suggest that the modulation constraint has more of a negative effect when using QAM signaling than when using QPSK signalling, at least for the parameters considered here.

Information outage probabilities for different rates were discussed in the previous chapter and shown in Fig. 3.6. More insight is provided by presenting the overall behavior of the normalized throughput efficiency against using different rates for hybrid-ARQ, as shown in Fig. 4.3. Table 4.1 summarizes the performance difference of the unconstrained Gaussian input against the QPSK constrained modulation by providing the required SNR for a throughput efficiency of $\eta_{e f f}=0.5$. When $R=1.25$, the loss due to modulation is low resulting in a difference of $0.23 \mathrm{~dB}$ between the unconstrained Gaussian input and the QPSK constrained modulation. At a rate of 1.5 , the loss due to modulation is $0.44 \mathrm{~dB}$, while at $R=1.75$ the difference is $0.87 \mathrm{~dB}$. These results show that when operating at higher code rates the system becomes more energy inefficient. This result is a manifestation of the higher probability of outage with the rate increase. 


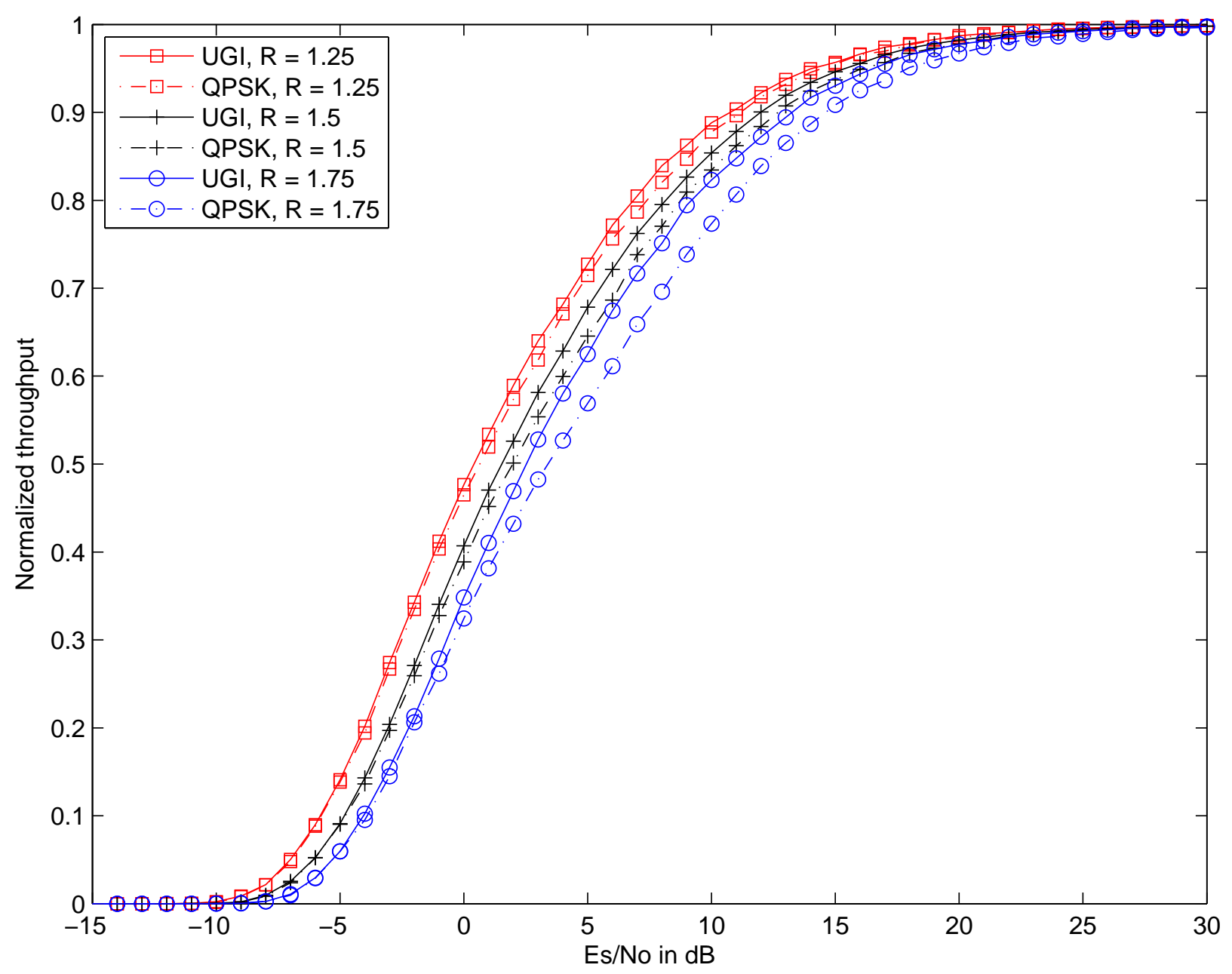

Figure 4.3: Throughput efficiency vs. SNR for unconstrained (solid line) and QPSK modulation (dashed line) in Rayleigh block fading using different rates; $R=1.25,1.5$, and 1.75.

\subsection{Conclusion}

When examining the throughput of any practical hybrid-ARQ system in block fading, there is always a loss relative to the information theoretic bounds derived by Caire and Tuninetti [3]. While modulation constraints cause a loss in diversity in FEC-coded block fading channels, there is no such diversity loss when using hybrid-ARQ. This chapter presented a methodology for determining the information theoretic throughput bound under modulation constraints, thereby allowing the relative throughput losses due to modulation to be quantified. The steps for determining the throughput of Hybrid-ARQ under modulation constraints included determining the AWGN capacity under modulation constraints 
then finding the information outage probability, and finally determining the throughput. While the purpose of this chapter has been to examine the effects of modulation constraints upon the theoretical throughput limits of hybrid-ARQ, the results can be compared against the actual performance achieved when using a practical channel code. In turn, this will quantify the loss due to coding. This is the topic of the following chapter. 


\section{Chapter 5}

\section{Channel Coding}

\subsection{Introduction}

After Shannon established the fundamental limits on the transmission rates in digital communication systems, much research has been invested in finding coding techniques that would approach the capacity limit. In 1993, Berrou, Glavieux, and Thitimajshima in [29], introduced a great breakthrough by proposing turbo codes which virtually closed the gap between the capacity limit and the actual performance achieved with a code. This chapter presents a discussion of error correcting codes that are key to realizing error-free communication through the use of appropriate redundancy.

Before delving into turbo error control coding, it is appropriate to mention two other main channel codes, namely, block codes and convolutional codes. Each code is characterized by the ratio $R$ of data bits to total bits $R=\frac{K}{N}$ called the code rate. Another important criteria is the coding gain, which emphasizes the reduction in the required $E_{b} / N_{0}$ to achieve a specified bit error rate (BER) of an error-correcting coded system relative to uncoded modulation.

\subsubsection{Block Codes}

A block code encodes a set of $K$ data bits into a code word of $N$ bits $(N>K)$. For each distinct sequence of $K$ data bits, there is a distinct codeword of $N$ bits. The performance of block codes is dominated by the minimum (Hamming) distance $d_{\min }$. The minimum 
Hamming distance $d_{\min }$ among all pairs of distinct codewords in a block code $\mathcal{C}$ is,

$$
d_{\text {min }}=\min _{\mathbf{v}_{i}, \mathbf{v}_{j} \in \mathcal{C}}\left\{d_{H}\left(\mathbf{v}_{i}, \mathbf{v}_{j}\right): \mathbf{v}_{i} \neq \mathbf{v}_{j}\right\}
$$

A code $\mathcal{C} \subset \mathcal{V}_{n}$, Hamming space of length n, with $|\mathcal{C}|=2^{k}$ and minimum distance $d_{\text {min }}$ is referred to a $\left(N, K, d_{\text {min }}\right)$ code for short. A code $\mathcal{C}$ is linear if the sum of every pair of codewords $\mathbf{v}_{i}, \mathbf{v}_{j} \in \mathcal{C}$ is also a codeword, $\mathbf{v}_{i}+\mathbf{v}_{j} \in \mathcal{C}$. For linear codes, the minimum distance is the minimum Hamming weight of all the nonzero codewords in $\mathcal{C}$,

$$
d_{\min }=\arg \min _{\mathbf{v} \in \mathcal{C}}\left\{w_{H}(\mathbf{v})\right\}
$$

\subsubsection{Convolutional Codes}

In convolutional coding, [30] and [31], the coded sequence of $N$ bits depends not only on the $K$ data bits but also on the previous $\nu-1$ data bits $(\nu>1$, where $\nu$ is called the constraint length). The encoding is done on a continuous basis on a stream of data rather than independent sets of $K$ data bits. Thus, convolutional codes are useful for realtime applications because they can be continuously encoded and decoded. A convolutional encoder with constraint length $\nu$ consists of a $\nu-1$ stage shift register. The message bits are applied serially to the input of the shift register, such that the output is a linear combination of the inputs and the contents of the shift register. In practice, the length of the input stream is often limited, after which the encoder is returned to the all zeros state prior to encoding the next frame by inserting tail bits (i.e. by feeding $\nu-1$ zeros into the encoder). A Recursive Systematic Convolutional (RSC) encoder, is constructed from a standard convolutional encoder by simply feeding back one of the outputs. An example constraint length $\nu=4$ RSC encoder can be seen in the circled part of Fig. 5.1.

Because the convolutional encoder is a finite state machine, it can be represented by a state diagram. A trellis diagram, which is used in the decoding process, is an expansion of the state diagram that explicitly shows the passage of time. The trellis shows each state at every instant of time. The importance of the trellis arises from its structure where each distinct input message stream and its corresponding output bits are represented by a unique path through the trellis. A sample of a trellis diagram for a 4 state convolutional code is 


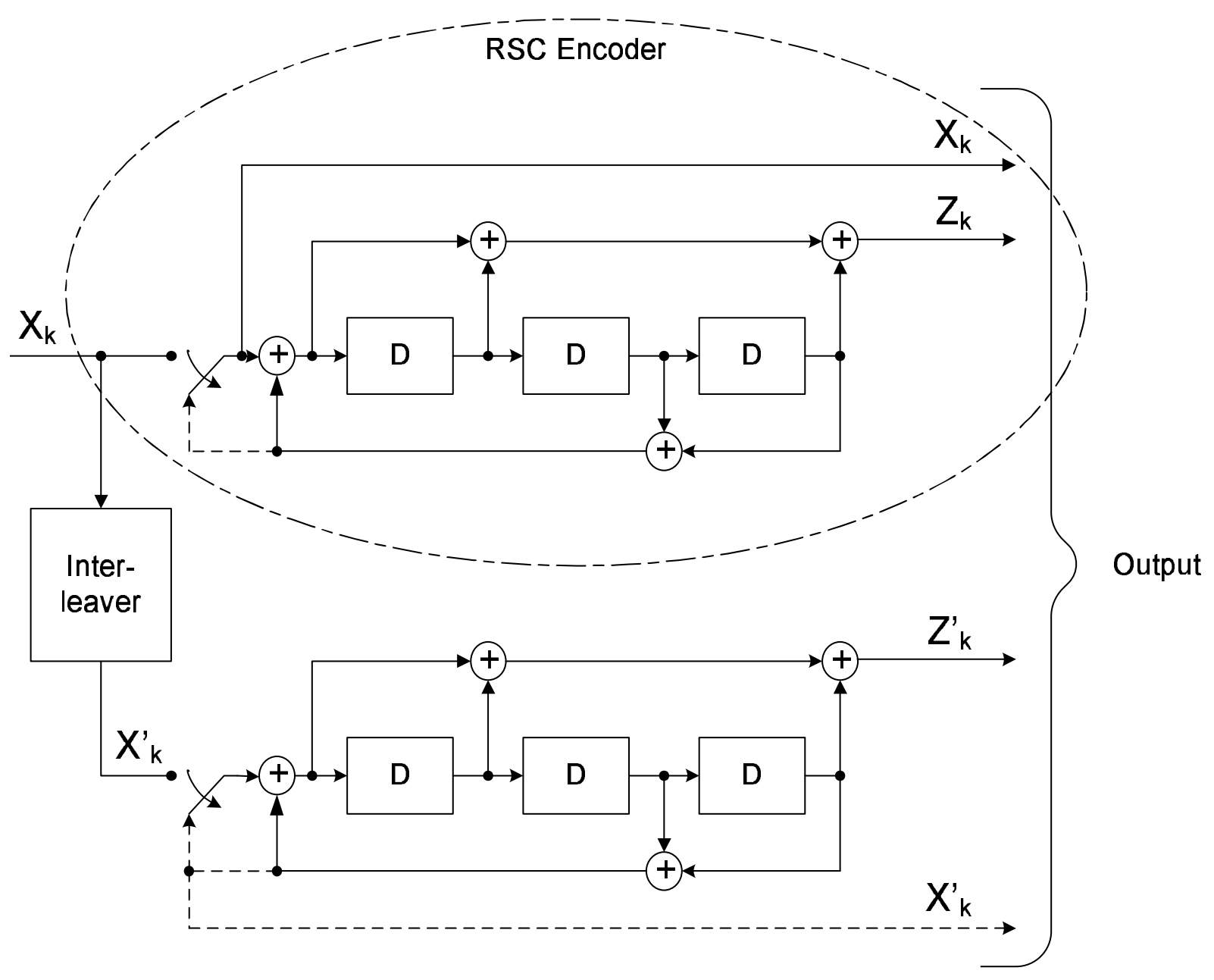

Figure 5.1: UMTS Turbo Encoder

shown in Fig. 5.2. Although not shown, the convention is to label each branch with input bit(s) and its corresponding output bits.

In [32], Viterbi derived a decoding algorithm that operates over the code trellis and provides a Maximum Likelihood (ML) decoding with low complexity and ease of implementation in hardware. The Viterbi algorithm, as it has come to be known, compares the received sequence with the possible transmitted sequences one trellis stage at a time. The Viterbi algorithm traverses the trellis by choosing the path through the trellis whose coded sequence differs from the received sequence in the fewest number of places according to some distance metric (e.g. Hamming distance for hard decision decoding, and squared Euclidean distance for soft decision decoding). When a new path is chosen, all paths leading into the 

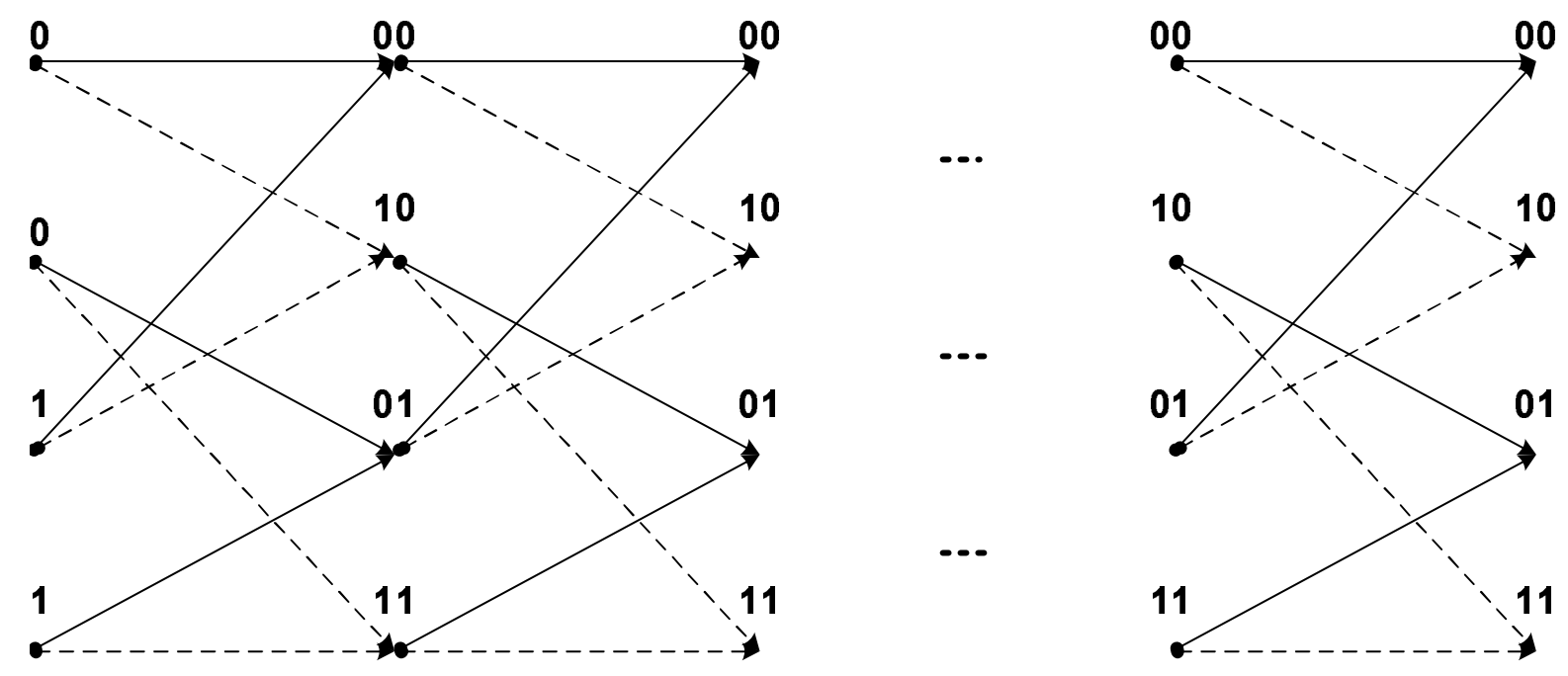

Figure 5.2: A trellis for a 4 state convolutional code. A solid line denotes a transition due to a binary input symbol 0 , where the dashed line represents the transition due to a binary input symbol 1

state other than the one that minimizes the distance can be discarded. Once a valid path is selected as the correct path, the decoder can recover the input data bits by reading them off the most likely path through the trellis.

\subsubsection{Turbo Codes}

An example of of a turbo encoder is shown in Fig. 5.1. This example shows the turbo encoder for the UMTS standard which uses two constraint length $\nu=4$ RSC encoders concatenated in parallel and an internal interleaver [8]. $X_{k}, 1 \leqslant k \leqslant K$, is the input to the first RSC constituent encoder and to the turbo code internal interleaver, where $K$ is the number of bits taking on values between $40 \leq K \leq 5114$. $Z_{k}$ is the output of the first RSC encoder. $X_{k}^{\prime}$ is the input to the second RSC encoder after having been interleaved, and $Z_{k}^{\prime}$ is the output. The exact operation of the interleaver is described in the UMTS standard [8] and is not repeated here. 


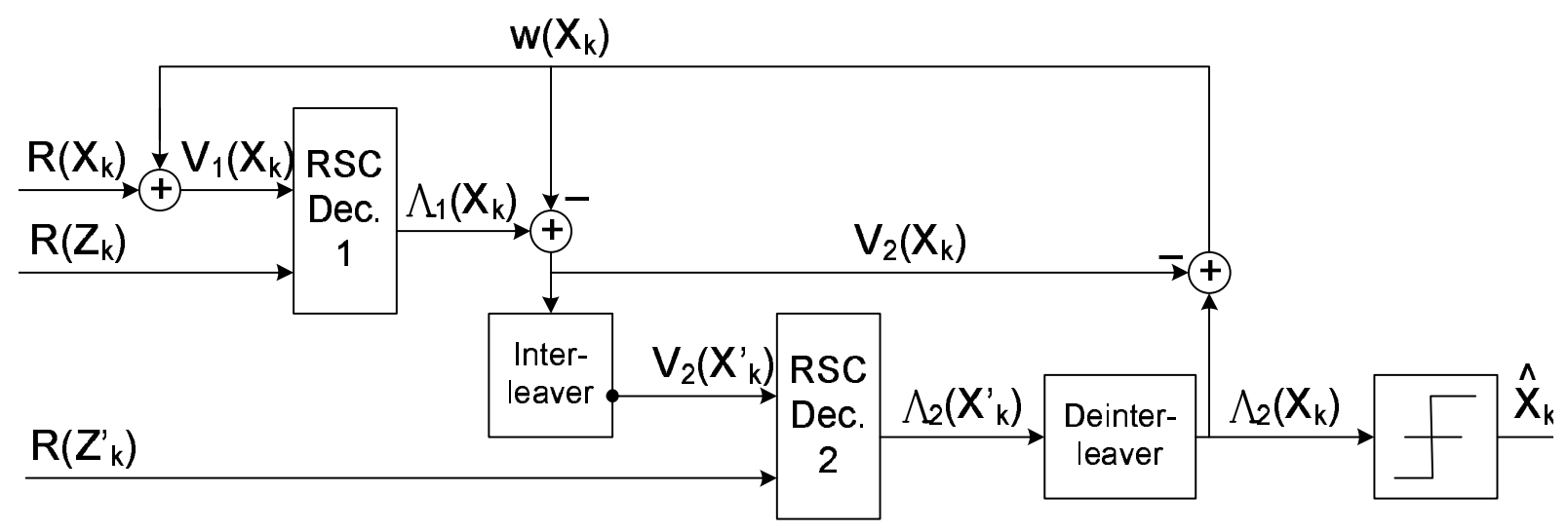

Figure 5.3: UMTS Turbo Decoder

\section{Decoder}

One implementation for the turbo decoder for the UMTS standard is shown in Fig. 5.3. $w\left(X_{k}\right)$ is the extrinsic information produced by the second decoder, which combined with the received systematic data in Log Likelihood Ratio (LLR) format form the first input to the upper RSC decoder. The second input to the upper decoder is the received parity bits in LLR format, $R\left(Z_{k}\right)$. The upper RSC decoder outputs a new LLR variable denoted $\Lambda_{1}\left(X_{k}\right)$. The inputs to the lower decoder are the sum of the systematic channel LLR and interleaved extrinsic information from the upper decoder, $V_{2}\left(X_{k}^{\prime}\right)$, and the interleaved received parity bits in LLR format, $R\left(Z_{k}^{\prime}\right) . \Lambda_{2}\left(X_{k}\right)$ is the deinterleaved LLR output of the lower RSC decoder, which the data estimate $\hat{X}_{k}$ is based on. The presence of a feedback loop implies an iterative manner of operation. Each of the RSC decoders shown operate during each half iteration, thereby alternating between each other. The RSC decoders each execute a version of the classic MAP algorithm that is implemented in the log-domain, ([33] and references therein). [33] explains that the log-domain MAP algorithm is based on the Viterbi algorithm, though with two alterations:

1. The trellis is swept twice, once in the forward direction and once in the reverse direction. 

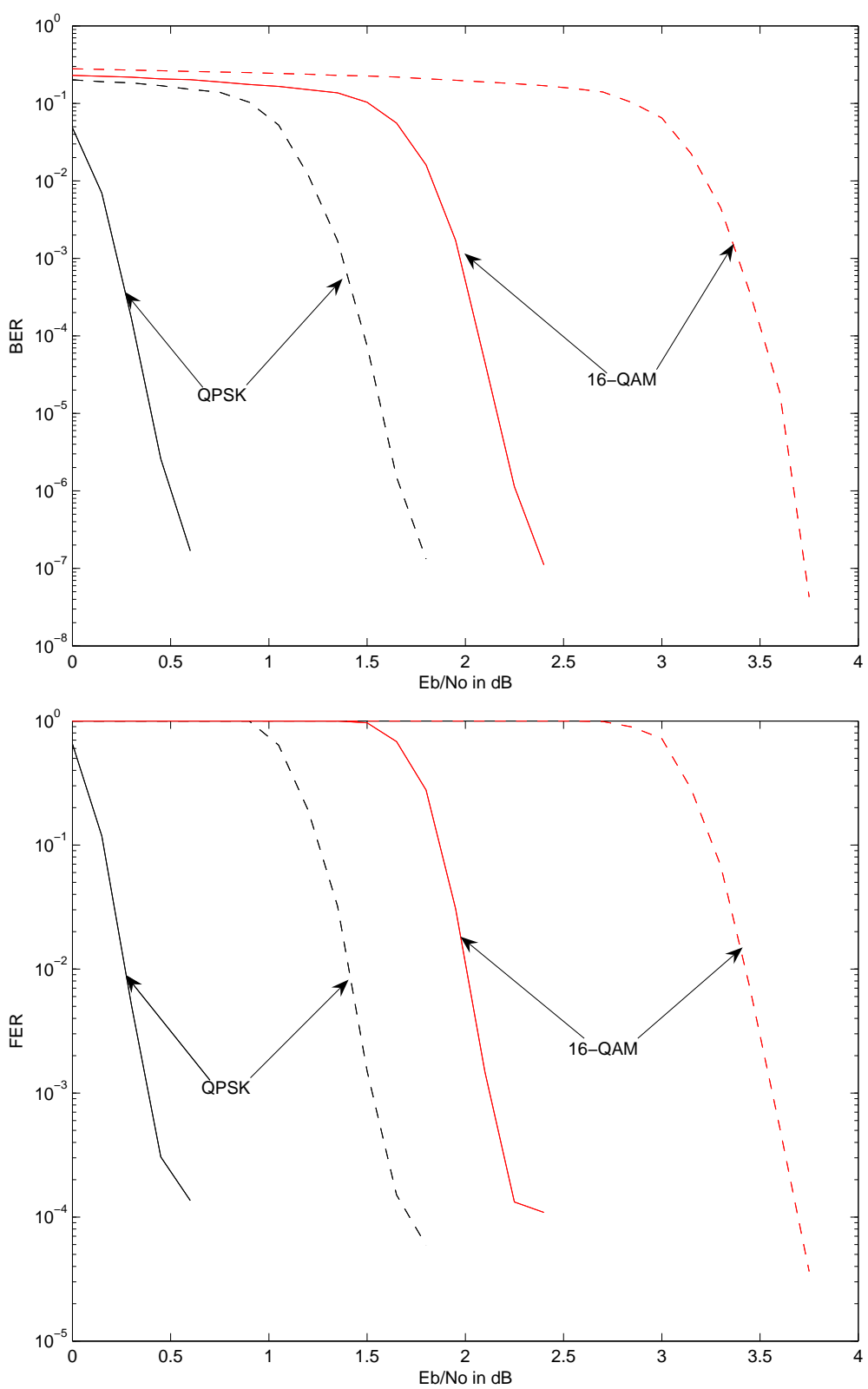

Figure 5.4: Simulation results for a UMTS rate $\frac{1}{3}$ Turbo code in AWGN (solid line) and Rayleigh fading (dashed line) for gray-labeled 16QAM signal with K $=5114$ after 14 iterations using the log-map algorithm (a) BER (b) FER

2. The add-compare-select (ACS) operation of the Viterbi algorithm is replaced with the Jacobi logarithm, also known as the max* operator. 
Table 5.1: Comparison of turbo code performance against AWGN and ergodic Rayleigh channel capacity $\left(B E R=10^{-5}\right)$ at rate $\frac{1}{3}$ in terms of $\frac{E_{b}}{N_{o}}$ under modulation constraints

\begin{tabular}{|c||c||c||c||c|}
\hline & AWGN Capacity & AWGN turbo & Ergodic Rayleigh Capacity & Rayleigh turbo \\
\hline QPSK & $-0.49 \mathrm{~dB}$ & $0.40 \mathrm{~dB}$ & $0.48 \mathrm{~dB}$ & $1.58 \mathrm{~dB}$ \\
\hline 16QAM & $1.11 \mathrm{~dB}$ & $2.16 \mathrm{~dB}$ & $2.38 \mathrm{~dB}$ & $3.61 \mathrm{~dB}$ \\
\hline
\end{tabular}

The Jacobi logarithm, (i.e. the max* operator), is computed as follows:

$$
\begin{aligned}
\max *(x, y) & =\log \left(e^{x}+e^{y}\right) \\
& =\max (x, y)+\log \left(1+e^{-|y-x|}\right) \\
& =\max (x, y)+f_{c}(|y-x|)
\end{aligned}
$$

Fig. 5.4 provides bit error rate (BER) and frame error rate (FER) results for the UMTS rate $\frac{1}{3}$ Turbo code in AWGN and Rayleigh fading for gray-labelled 16-QAM signal with $K=5114$ after 14 iterations using the log-MAP algorithm. From the figure, one can observe the fact that turbo codes have very good performance for low signal-to-noise channel conditions. A comparison of the turbo code performance against the AWGN and ergodic Rayleigh capacity curves in Figs. 3.2 and 3.3 for QPSK and gray-labelled 16QAM is shown in Table 5.1 for rate $\frac{1}{3}$ and at a $B E R=10^{-5}$. The difference in performance achieved when using a turbo code against the capacity curve in terms of $\frac{E_{b}}{N_{o}}$ is 0.89 and $1.1 \mathrm{~dB}$ for QPSK modulation for the AWGN and ergodic Rayleigh channels, respectively. As for 16QAM, the difference is 1.05 and $1.23 \mathrm{~dB}$.

\subsection{Limits on the Throughput of HSDPA}

High Speed Downlink Packet Access (HSDPA) is a newly standardized hybrid-ARQ protocol that uses QPSK and (gray-labelled) 16QAM bit interleaved turbo-coded modulation. In this section, the throughput efficiency of HSDPA (obtained through computer simulations and numerical analysis) is compared against the corresponding information theoretic bounds (both unconstrained and modulation-constrained).

With HSDPA, the message is first encoded by the rate $1 / 3$ UMTS turbo code shown in 
Table 5.2: Maximum Throughput (kbps) for the Fixed Reference Channel Definitions: H-Set $1,2,3$

\begin{tabular}{|c||c||c|}
\hline & QPSK & 16-QAM \\
\hline H-Set 1 & 534 & 777 \\
\hline H-Set 2 & 801 & 1166 \\
\hline H-Set 3 & 1601 & 2332 \\
\hline
\end{tabular}

Fig. 5.1. A two stage rate matching algorithm is used to puncture the codeword prior to QPSK or 16QAM modulation. Because the encoder is binary and separated from the M-ary modulator by a bitwise interleaver, this is an example of bit-interleaved coded-modulation (BICM) [10]. After bitwise interleaving the codeword, it is modulated using either QPSK or 16-QAM. For each modulation type, there are eight ways to perform rate matching, which is specified by a three bit variable called the redundancy version [34]. In the case of 16-QAM, gray-labelling is used and rate matching can be used to essentially rearrange the signal constellation mapping. When a retransmission is requested, the rate matching algorithm can either be run with the same redundancy version, resulting in a repetition code which is diversity-combined at the receiver, or a different redundancy version can be used for each transmission, in which case code-combining is used. Blocks continue to be generated by rate matching with different parameters and sent until either the destination correctly decodes the message or an upper limit on the number of retransmissions is reached.

Monte Carlo simulations of HSDPA were performed using key parameters, such as the message size $(K)$, block length after rate matching $(L)$, sequence of redundancy versions, and time between the start of consecutive blocks $(\tau)$; chosen to comply with the 3GPP approval standard [34]. There are a total of six test sets defined in [34], termed H-Set 1 through H-Set 6. In this section, we give throughput results for H-Set 1 through 3, which differ only in the value of $\tau$. The time between the start of consecutive blocks is $\tau=6,4$, and 2 msec for H-Set 1, 2, and 3, respectively. The maximum throughput for these three H-Sets, which occurs as $E[B] \rightarrow 1$, or equivalently as $\mathcal{E}_{s} / N_{o} \rightarrow \infty$, is given in Table 5.2. For a plot of the maximum throughput of H-Set 1 refer to Appendix B.

Fig. 5.5, which replicates Fig. 4.2 results' with the addition of the HSDPA results, shows throughput efficiency versus SNR in Rayleigh block fading for H-Sets 1 through 3 


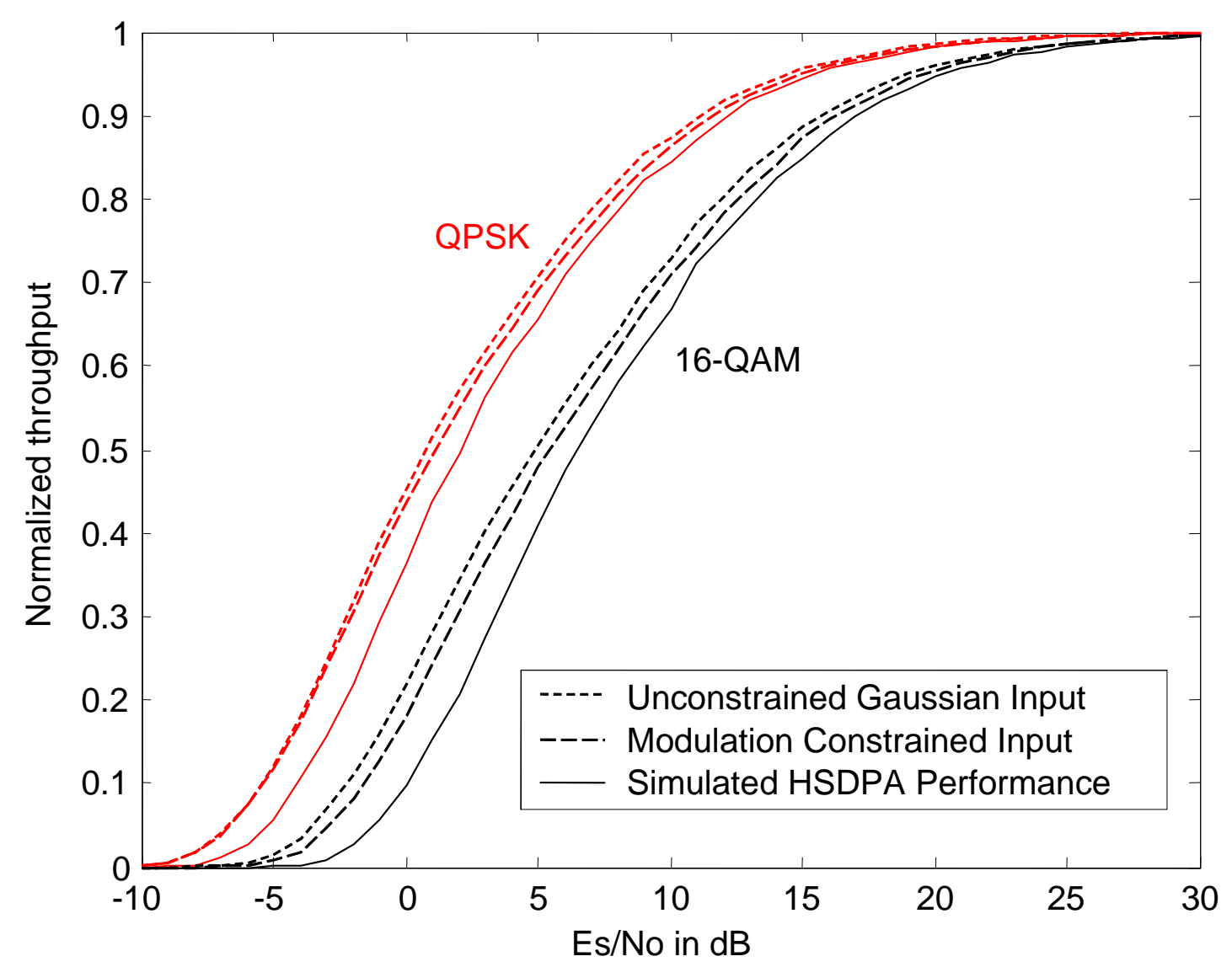

Figure 5.5: Throughput efficiency of HSDPA H-Sets 1 through 3 in Rayleigh block fading using QPSK or 16-QAM modulation. For each modulation type, the unconstrained and modulation-constrained theoretical limits are compared against the simulated performance of the HSDPA system.

using both QPSK and 16-QAM modulation. Since H-Sets 1 through 3 differ only in the value of $\tau$, all three have the same throughput efficiency. The figure shows two groups of three curves. The group on the left is for QPSK and the group on the right is for 16-QAM. For each modulation type, three curves are shown. The leftmost curve is the informationtheoretic limit on throughput with an unconstrained (i.e. Gaussian-distributed) input, while the middle curve is the information-theoretic limit with a modulation-constrained input. The rightmost curve is the throughput of the simulated HSDPA system in block fading.

The results shown in Fig. 5.5 indicate how much of the performance difference between HSDPA and the corresponding theoretical limits is due to the modulation constraints and 
how much is due to the use of the turbo code. For instance, with QPSK, a throughput efficiency $\eta_{\text {eff }}=0.5$ is achieved at $\mathcal{E}_{s} / N_{0}=0.77,1.12$, and $2.05 \mathrm{~dB}$ for the unconstrained, modulation-constrained, and actual HSDPA cases, respectively. This implies that while HSDPA has a $1.28 \mathrm{~dB}$ loss compared to the unconstrained theoretic bound, about 0.97 $\mathrm{dB}$ of this loss can be attributed to the turbo code, while the rest is attributed to the modulation constraint. Similarly, for 16-QAM, a throughput efficiency $\eta_{\text {eff }}=0.5$ is achieved at $\mathcal{E}_{s} / N_{o}=4.88,5.44$, and $6.48 \mathrm{~dB}$ for the unconstrained, modulation-constrained, and actual HSDPA cases, respectively. This indicates that of the $1.60 \mathrm{~dB}$ difference between HSDPA and the unconstrained theoretic bound, about $1.04 \mathrm{~dB}$ of this loss is due to the turbo code.

\subsection{Conclusion}

There is always a relative loss in throughput performance of any practical hybrid-ARQ system in block fading compared to the information theoretic bounds. In the case of HSDPA, this loss is in the range of $1-2 \mathrm{~dB}$. While there are several causes for this loss, these causes can be roughly partitioned into those that are due to the modulation constraints and those that are due to the use of a practical code. In the case of HSDPA, about 0.5 to $0.6 \mathrm{~dB}$ of the loss is due to using a 16-QAM modulation constraint, while up to $0.4 \mathrm{~dB}$ of the loss is due to using QPSK modulation constraints. In the case of HSDPA, the coding loss is about $1 \mathrm{~dB}$ when using either 16-QAM or QPSK modulation constraints.

As for the losses due to causes other than modulation, there are several factors. First, both the unconstrained and modulation-constrained throughput bounds were found by using expressions for the AWGN Shannon-sense capacity of each block. As such, these expressions are derived under the assumption of an infinite block length. However, practical systems must use a finite block length (e.g. in HSDPA it is 2400 QPSK symbols or 1920 QAM symbols). Thus some of the loss is due to finite block length effects, and the amount of this loss can be determined using an extension of the sphere-packing approaching described in [35]. Another issue with HSDPA is that while the rate matching algorithm can be used to produce up to eight distinct blocks for each modulation type, these blocks are not mutually exclusive, i.e. some code bits will appear in more than one block. As a consequence, the 
processing at the receiver will actually be a combination of code-combining and diversitycombining. This problem can be alleviated by using a rate compatible code, such as a rate compatible turbo code [36], which will have distinct blocks and is therefore amenable to pure code-combining. One weakness of using rate compatible coding is that it imposes a finite upper limit on the maximum number of retransmissions $B_{\text {max }}$; this drawback can possibly be alleviated by using a rateless code such as an LT code [37] or a Raptor code [38]. In addition to finite block length effects and presence of repeated code bits, the other losses relative to the information theoretic bounds can be attributed to the code imperfectness as defined by [39]. 


\section{Chapter 6}

\section{Hybrid-ARQ Based Relay Networks}

\subsection{Introduction}

In the previous chapters, point to point communication was assumed along with the traditional hybrid-ARQ protocol where the source always initiates the transmission of data. The focus of this chapter is the extension of hybrid-ARQ to relay networks and the impact of modulation constraints upon such systems. In a relay network, additional relay terminals assist the transmission of the message from source to destination. While the initial hybridARQ transmission must always come from the source, each retransmissions may come from any relay that overhears the message. The destination then combines the information from all the received transmissions be it via the source or relay/s. Thus the time-diversity benefits of hybrid-ARQ are combined with the spatial-diversity of relaying.

Relays are classified into two categories according to the way they enact their retransmissions [15]:

1. Amplifying relays (amplify and forward): the relay simply amplifies the source's transmission and retransmits.

2. Decoding relays (decode and forward): the relay decodes the source's transmission, re-encodes and retransmits.

From here onwards, decoding relays are assumed in the analysis presented in this chapter. 
Many relaying strategies can be employed to place constraints on how the nodes in the network cooperate. In the case of no constraints and where all nodes can simultaneously transmit together, coherent detection at the receiver and co-phasing of signals by transmitting nodes proves difficult as nodes use different local oscillators and current cost-effective technology trends are not up to par to deal with such complexities. The literature includes many constrained network cooperation schemes (e.g. [15], [40]). A trivial case would be the conventional multi-hop, where a routing protocol manages point to point links to deliver the message, however, multi-hop fails to make use of the spatial diversity available in the network introduced by the broadcasting nature of wireless communication.

When relaying is used, a key issue is how to select the relay. In [41], a relay selection scheme is proposed based on the geographic distribution of nodes in the network. Basically, the node closest to the destination is selected to act as the relay. In an isotropic propagation environment, this is equivalent to selecting the node whose link to the destination has the highest average SNR. Another option in a block fading environment is to select the node whose link to the destination has the highest instantaneous SNR [42]. However, [41] fails to make use of transmit diversity present in the network since nodes flush their memory from any previous transmissions once a relay is decided upon after the contention scheme (i.e. information can not be combined at the destination from multiple transmissions). [11], which is the emphasis of this chapter, instead does make use of the transmit diversity by combining the information from the transmissions at each node. While the results in [11] assumed an unconstrained channel input, the results presented in this chapter show the impact of modulation constraints on hybrid-ARQ relaying protocols.

Fig. 6.1 shows a simple example of a relay network with a source, a destination and one relay. Single channel outages will occur in the network if any of the channels' instantaneous capacities is less than the code rate per block, or $C\left(\gamma_{s, r}\right), C\left(\gamma_{s, d}\right), C\left(\gamma_{r, d}\right) \leq R_{B}$. For this simple relay network, the overall relay channel outage will occur if any of the following scenarios occurs:

1. The source-relay link is in outage $C\left(\gamma_{s, r}\right) \leq R_{B}$ along with source-destination link $C\left(\gamma_{s, d}\right) \leq R_{B}$ 


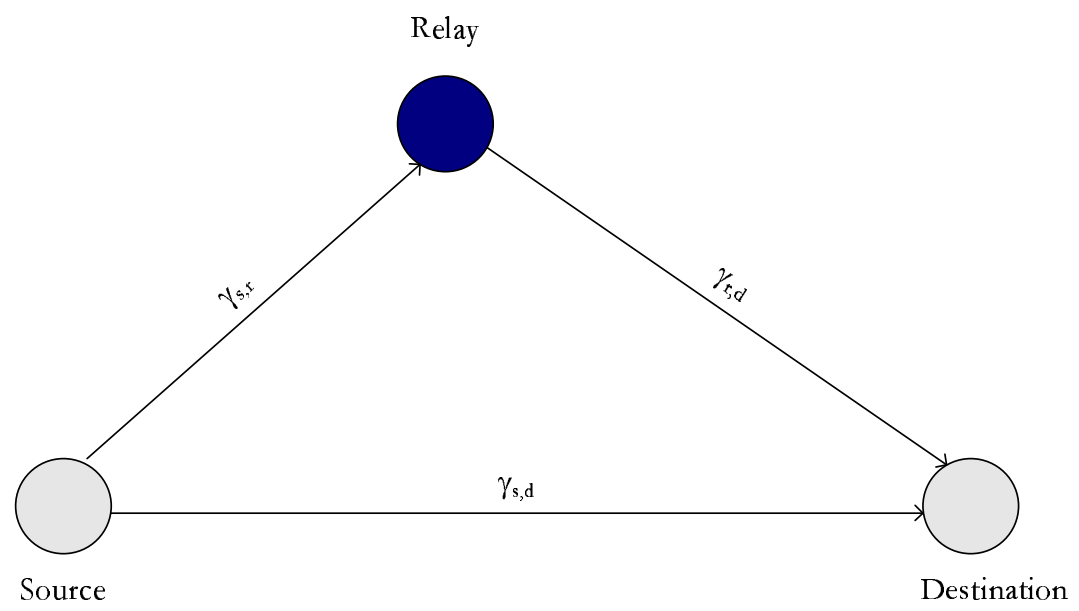

Figure 6.1: A simple relay network

2. The source-destination link is in outage $C\left(\gamma_{s, d}\right) \leq R_{B}$ along with relay-destination link $C\left(\gamma_{r, d}\right) \leq R_{B}$

\subsection{Relay Network Model}

The general relay network model can be presented as shown in Fig. 6.2. The only difference from the point-to-point model is that the transmitted output of the M-ary modulator can be overheard by any node within the broadcast range. The modulated codeword is broken into $B_{\max }$ equal-length blocks, where the $b^{\text {th }}$ block is transmitted with an instantaneous SNR denoted by $\gamma_{b, i \rightarrow j} \equiv\left|h_{i \rightarrow j}[b]\right|^{2} \mathcal{E}_{s} / N_{0}$, where $b, i \rightarrow j$ denotes the instantaneous SNR between the transmitting node $i$ and the receiving node $j$ during the $b^{\text {th }}$ block transmission. All transmissions of signals occur over a block fading channel such that each block is sent over an independent channel. This is denoted by the fading coefficient $h_{i \rightarrow j}[b]$, for the link between nodes $i$ and $j$ of the $b^{\text {th }}$ block, being constant for the entire duration of the block; here the channel during one block is conditionally Gaussian (conditioned on $h_{i \rightarrow j}[b]$ ).

Following the notation used in [11], the relay network is composed of a cluster of nodes $\mathcal{N}=\left\{Z_{k}: 1 \leq k \leq K_{n}\right\}$ consisting of a source $Z_{s}=Z_{1}$, a destination $Z_{d}=Z_{K_{n}}$, and $K_{r}=K_{n}-2$ relays. The numbering of relays refers to their respective distance from the destination, where $Z_{K_{n}-1}$ is the closest to the destination. Each node knows its geographical 


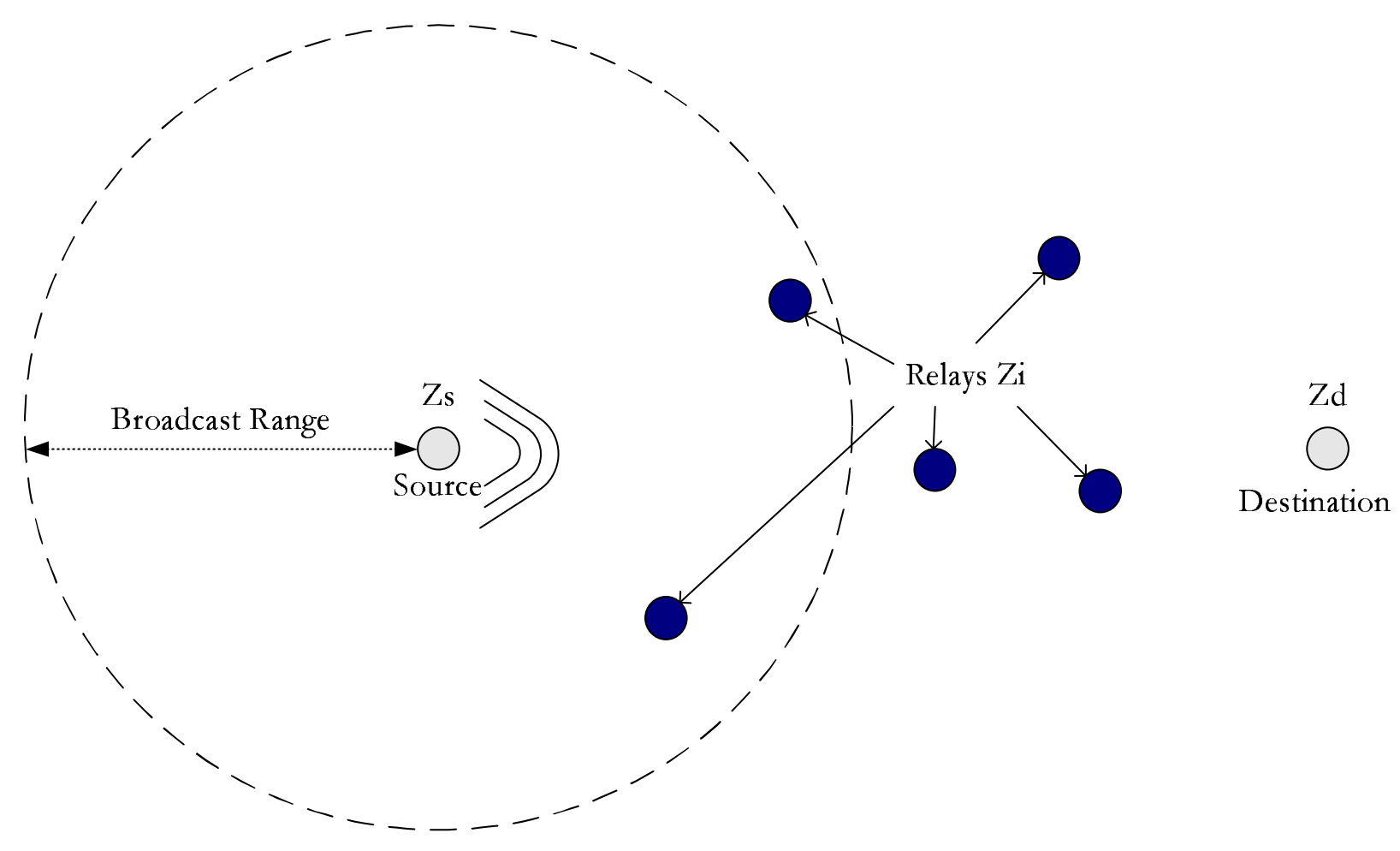

Figure 6.2: A cluster of $\mathrm{K}$ nodes constituting a relay network

location compared to other nodes (e.g. via an on-board Global Positioning System GPS) and is equipped with a single antenna and a single half-duplex radio, hence, when a node in $\mathcal{N}$ begins transmission the only nodes that can overhear the transmission are other nodes in $\mathcal{N}$ that are not transmitting at the same time. Here, transmission over orthogonal time slots $s$ where each block occupies one time slot is assumed.

The source's transmitter sends the first block $\mathbf{x}[1]$ at time $s=1$, and if the destination's receiver is able to successfully decode it, an acknowledgement will be sent back through a feedback channel (we assume here that the feedback channel is error and delay-free and that an ideal error detecting code allows the receiver to discriminate between correctly and incorrectly decoded messages). If the source's transmitter receives an acknowledgement, it will move on to the next message; otherwise, in contrast to the point to point model, any node that overheard the transmission and was able to successfully decode it can now retransmit the message instead in the next time slot. Geographical relaying, whereby the node that is closest to the destination, is used to determine which node of the decoding set acts as the 
next relay. Thereafter, if the source has not received an acknowledgement, the node in the decoding set closest to the destination sends the next block (i.e. not necessarily the source). This process continues until either the message is received correctly by the destination or the last $\left(B_{\max }\right)$ block is transmitted.

As stated in earlier chapters, the fading coefficient $h_{i \rightarrow j}[b]$ is random, then so is the instantaneous $S N R$ of the $b^{\text {th }}$ block, and therefore so is the corresponding capacity $C\left(\gamma_{b, i \rightarrow j}\right)$. For Rayleigh block fading, $\left|h_{i \rightarrow j}[b]\right|$ is Rayleigh and $\left|h_{i \rightarrow j}[b]\right|^{2}$ is exponentially distributed. When incremental redundancy and code-combining is used, then after the $B^{\text {th }}$ block has been transmitted, the capacities of the $B$ blocks add, since each block is sent over an independent Gaussian channel. The resulting capacity for node $j$ after block $B$ has been transmitted in a relay network is:

$$
C_{j}(B)=\frac{1}{B}\left(\sum_{b=1}^{B} C\left(\gamma_{b, i \rightarrow j}\right)\right),
$$

where $i$ is the index of the node that transmits during block $b$ and where the $\frac{1}{B}$ term is needed because blocks are orthogonal and therefore effectively occupy only $1 / B^{\text {th }}$ of the channel.

For repetition coding and diversity combining the SNRs add and so the capacity when $B$ blocks are transmitted is:

$$
C_{j}(B)=\frac{1}{B} C\left(\sum_{b=1}^{B} \gamma_{b, i \rightarrow j}\right) .
$$

Recall that when there are no modulation constraints, the capacities in (6.1) and (6.2) are found from the unconstrained AWGN capacity (3.9), while when there are modulation constraints equation (3.10) or equations (3.12) and (3.13) must be used for CM and BICM, respectively.

Then the information theoretic bound on the FER in block fading for a single node $j$, as a function of $B$, is the information outage probability, defined as the probability that the instantaneous capacity of node $j$ is less than the rate $R_{B}=R / B$,

$$
p_{0, j}=P\left[C_{j}(B)<R_{B}\right]
$$

Whenever $C_{j}(B)<R_{B}$, an information outage occurs, and reliable signaling to that node is not possible. Denote $\mathcal{D}(s)$ as the decoding set at the beginning of time slot $s$ where nodes 
are added to the set as soon as they accumulate enough information to decode the message (i.e. $\mathcal{D}(s)=\left\{C\left(Z_{i}\right)>R_{B}\right\}$; where $R_{B}$ is the code rate per block). No node is taken out of the decoding set once it is added to it and the source is always included at $s=1$; (i.e. $\left.\mathcal{D}(1)=\left\{Z_{s}\right\}\right)$. Only one node is allowed to transmit in a given time slot. The overall outage probability is the probability that the destination is in outage (i.e. $C_{K_{n}}<R_{B}$ ), and hybrid-ARQ retransmission stop once $C_{K_{n}}>R_{B}$.

\subsection{Throughput Analysis}

The throughput analysis for hybrid-ARQ based relay networks follows directly from the point to point throughput analysis carried out in section 4.3. This section will then elaborate on the effect of path loss parameters and network topologies instead and show their respective throughput results through the use of Monte Carlo integration.

The parameters used for all the simulations are as follows. The distance between the source and the destination is normalized to one, and the relays' locations are scaled accordingly. Once again, all results assume the use of incremental redundancy and code combining. The block rate is $R=1.5$. Finally, the use of the term relaying gain, the difference in the SNR between two curves for a given throughput efficiency, is used in order to interpret the results.

\subsubsection{Path Loss Exponent Coefficient}

In order to study the effects of the path loss exponent coefficient, $\alpha$, on throughput, a linear practical relay network of 3 nodes was used (i.e. a source, a destination, and a relay placed halfway between them). The maximum number of transmissions is $B_{\max }=4$.

Fig. 6.3 demonstrates the effect of varying the path loss exponent coefficient. It can clearly be seen that at high $E_{s} / N_{o}$ the effect of the path loss exponent for both the QPSK constrained modulation and the unconstrained Gaussian input is the same. This is because at high SNR transmission is direct (i.e. without the use of a relay), and since distance from source to destination is unity the path loss does not depend on $\alpha$. The behavior seen for low SNR values seems counterintuitive, for as $\alpha$ increases the path loss increases as well. 


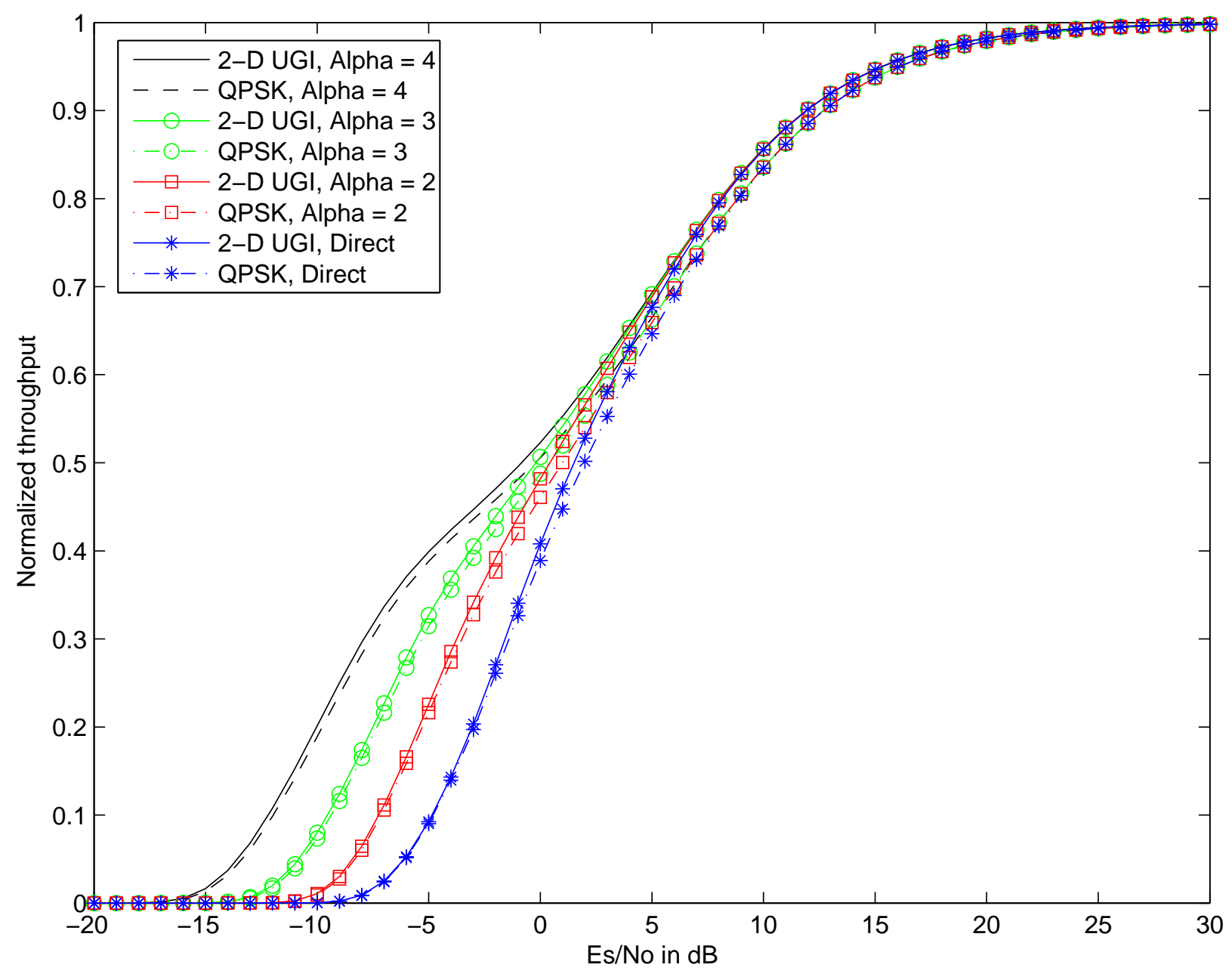

Figure 6.3: Throughput efficiency vs. SNR for unconstrained (solid line) and QPSK modulation (dashed line) in Rayleigh block fading using different values for the path loss exponent; $\alpha=2,3,4, K_{r}=1$

However, this suggests that there is more to gain with relaying in harsher channel conditions. This can be explained given the normalized distance between the source and the destination. For the normalization, the following relation was assumed: $\frac{k}{d^{\alpha}}=1$. This in turn results in a gain from the source to relay and relay to destination. For a relay placed halfway the relation becomes $\frac{k}{\left(\frac{d}{2}\right)^{\alpha}}=1$ which equals $2^{\alpha} \frac{k}{d^{\alpha}}=1$.

\subsubsection{Maximum number of Transmission, $B_{\max }$}

As mentioned in chapter 4, hybrid-ARQ does not keep on transmitting forever. For instance, as in the example of HSDPA, the maximum number of transmission, $B_{\max }$, was 4 . 


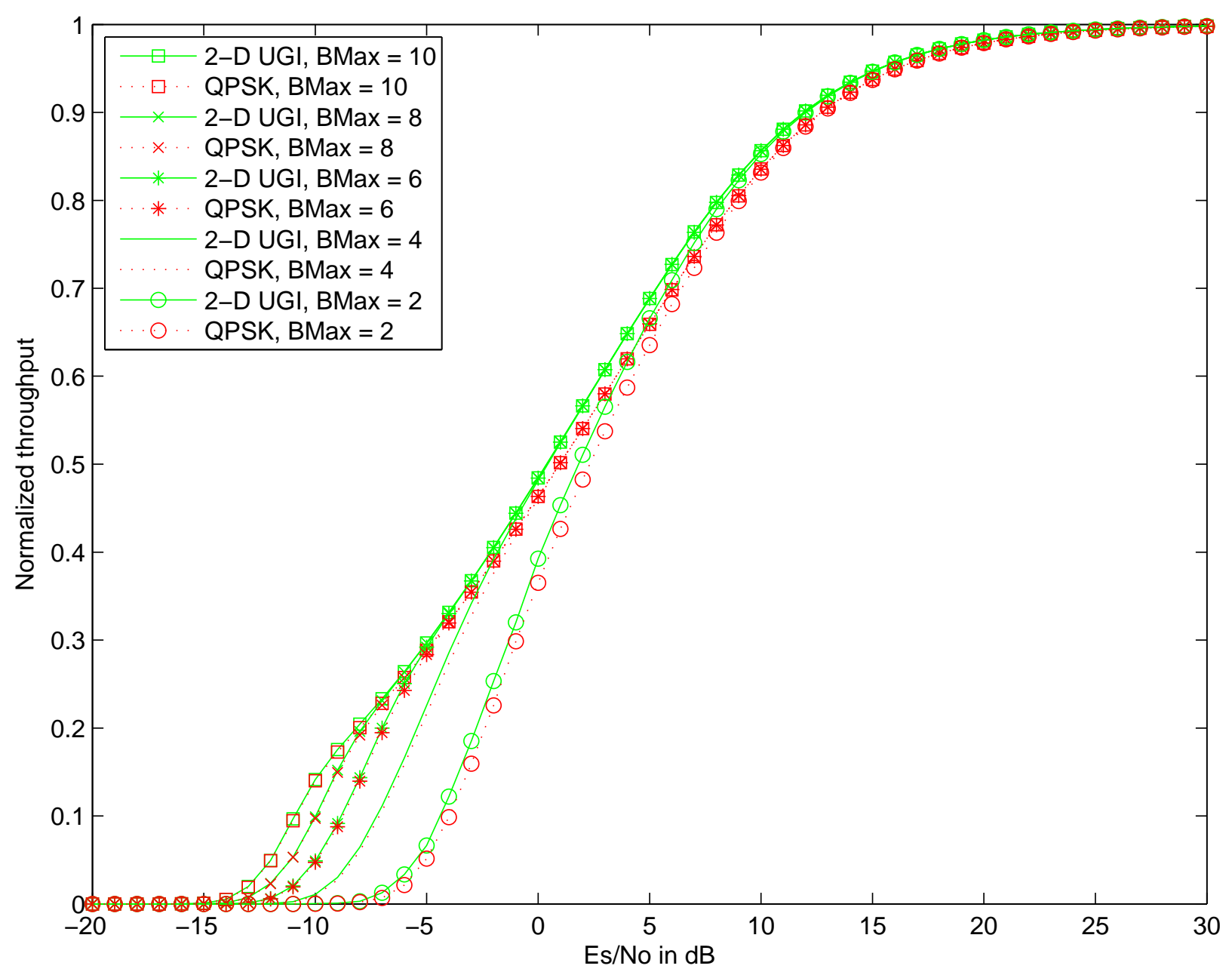

Figure 6.4: Throughput efficiency vs. SNR for unconstrained (solid line) and QPSK modulation (dashed line) in Rayleigh block fading using different values for the maximum number of transmission; $B_{\max }=2,4,6,8,10, K_{r}=1$

This section investigates other $B_{\max }$ values, however, one should note that the optimality of the system does not depend merely on the throughput performance. For instance, real-time applications require systems that provide little or no delay. The results were simulated for a linear practical relay network of 3 nodes where the relay was placed midway between the source and the destination. QPSK modulation was used with a path loss exponent $\alpha=2$.

As one would expect, Fig. 6.4 shows that as the number of maximum transmission increases, the throughput performance is significantly higher. Equation (6.1) explains this rather easily. The instantaneous capacity for any node is the sum over all the transmitted blocks since each block is sent over an independent Gaussian channel. Hence, as the number 
of transmissions increases then so would the instantaneous capacities which in turn results in lower probability of outage. Thus, providing better throughput measurements. The convergence of the curves at high SNR is due to the fact that the system behaves in a direct transmission fashion since the first block will always be decoded. Using larger $B_{\text {max }}$ is more beneficial at low SNR values, though that implies that the system must endure greater latencies. One more thing to note is that the law of diminishing returns applies here, that is as $B_{\max }$ increases the relaying gain gets smaller. For example, when the throughput efficiency is $\eta=0.2$, the transition from $B_{\max }=2$ to 4 has the highest relaying gain. Whereas the relaying gain in the transition from 4 to 6 is almost cut in half and so on.

\subsubsection{Relay Network Topology}

This section investigates the use of various relaying topologies. Whenever more than one relay is considered, the distances between the relays are assumed to be large enough that they experience independent fading. In addition to the parameters mentioned earlier, all the following plots were generated with a maximum number of transmission of $B_{\max }=4$, a path loss exponent $\alpha=2$, and QPSK constrained modulation where applicable.

\section{Relative Relay Distance}

Results for the 3 node linear topology with a source, a destination, and a relay were given before in one form or the other. However, the relay was always placed in the middle between the source and the destination. The plots in Fig. 6.5 provide insight to whether the relay should be placed closer to the source or closer to the destination. The plots are generated according to the ratio of the distance between the [relay to source : relay to destination].

In Fig. 6.5(a), the relay is closer to the source than it is to the destination. The throughput performance is worse when the relay is closest to the source, and improves as the relay moves further away. It is quite obvious that the highest relaying gain is achieved when the relay is exactly half way between the source and the destination. This is also true for the case of the relay being closer to the destination as can be shown in Fig. 6.5(b). However, if one was faced with a choice of whether to place the relay closer to the source or the destination, 

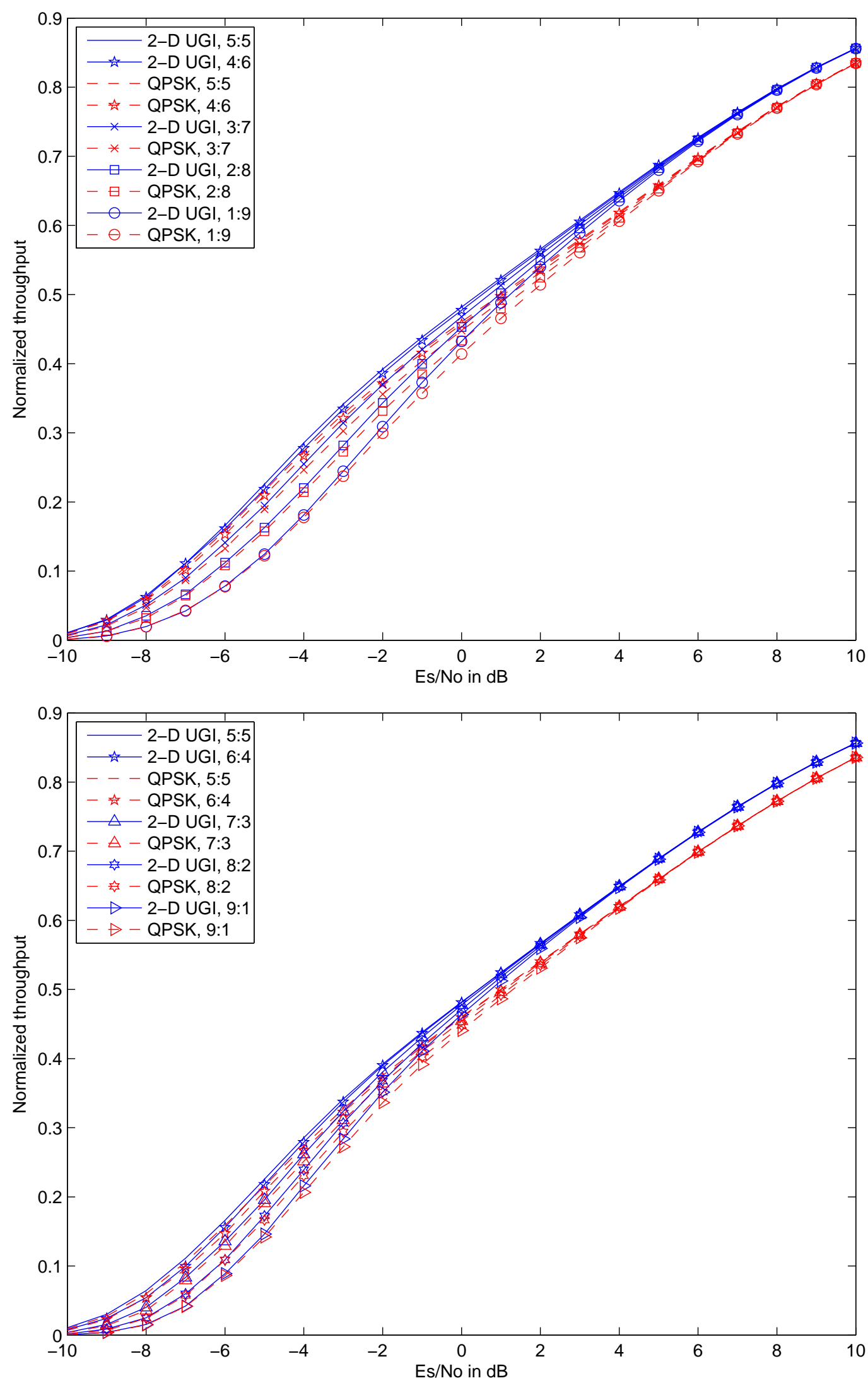

Figure 6.5: Throughput efficiency vs. SNR for unconstrained (solid line) and QPSK modulation (dashed line) in Rayleigh block fading for different relative relay distances, $K_{r}=1$. (a) Relay closer to source (b) Relay closer to destination 


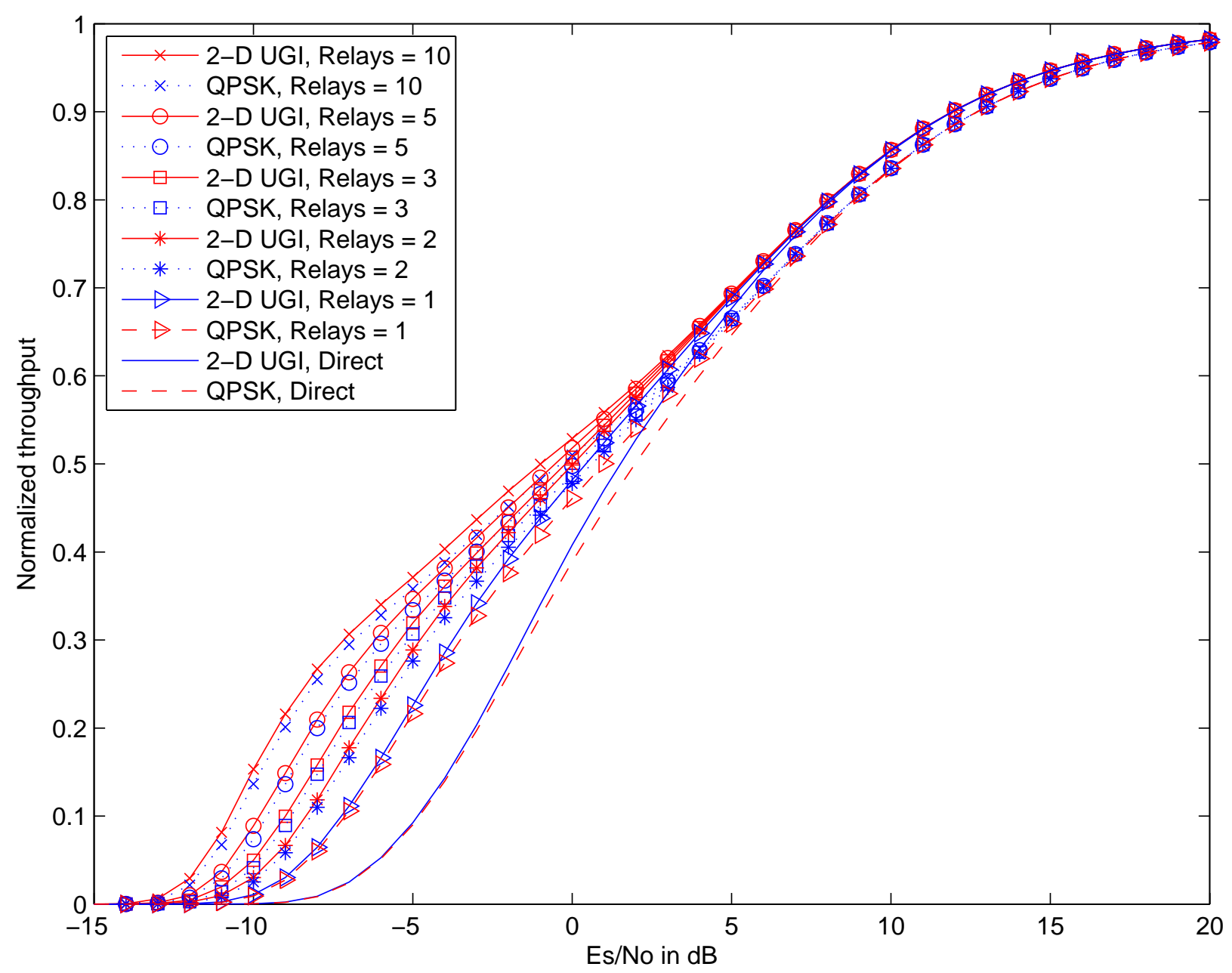

Figure 6.6: Throughput efficiency vs. SNR for unconstrained (solid line) and QPSK modulation (dashed line) in Rayleigh block fading using different number of relays; $K_{r}=1,2,3,5,10$ in an equidistant linear topology

one should opt for the latter. The plots do not clearly show that, though after a closer look positioning the relay closer to the destination provides better throughput performance and higher relaying gains when compared with the reciprocal ratio. For example, at $\frac{E_{s}}{N_{o}}=-2$ $\mathrm{dB}$, the throughput efficiency for the relative relay distance of $9: 1$ is 0.35 and 0.34 for the unconstrained Gaussian input and the QPSK constrained modulation, respectively. For the relative relay distance of $1: 9$, the throughput efficiency is 0.31 for the unconstrained Gaussian input and 0.30 for the QPSK constrained modulation. 


\section{Equidistant Linear Topology with Varying Number of Relays}

To demonstrate the impact of the number of relays on the throughput performance, we consider a generalized line network where the relays are equally spaced between the source and the destination. The classic relay network with 1 relay is compared to networks with 2, 3, 5, and 10 relays. The performance depicted in Fig. 6.6 shows that the use of a larger number of relays provides a higher relaying gain at low SNR values. Once again the law of diminishing returns is quite obvious, for low SNR, in the increased deployment of relays in a wireless network. The transition from direct transmission to one relay results in the highest relaying gain. Thereafter, any transition to a network with a higher number of relays results in a more diminished relaying gain.

In [11], it was illustrated that there exists a practical upper limit on the size of a network. The two reasons cited were the fact that each node in the relay network must expend a nonnegligible quantity of energy to receive and process the message; therefore making it unwieldy for a large number of nodes since reception energy can then exceed the energy consumed by transmitting the RF signal. The second reason relates to deterioration of the bandwidth efficiency of the system with large networks which is partly due to the fact that nodes cannot transmit while listening to sent messages, hence channel resources are not quickly reused. The latter reason is further substantiated by the curves shown in Fig. 6.6. [11] suggested that a maximum limit of 10 nodes should be used in any relay network, or a maximum of 10 nodes in any cluster if the relay network consists of a group of clusters.

\subsection{Conclusion}

In order to take advantage of the spatial diversity that is intrinsic in the wireless broadcast environment, hybrid-ARQ based relay networks allow transmissions to originate from nodes other than the source. The capacities of a node in a relay network in block fading for the unconstrained and constrained modulation were given for both code and diversity combining based on the results derived in earlier chapters. Afterwards, results for the information outage probability for the block fading channel in a hybrid-ARQ based relay network were given. 
Using the results derived earlier for throughput, performance limits and numerical results were presented for some representative topologies and with certain constraints of practical relay networks. It was shown that with a higher path loss exponent coefficient, relay networks provide higher relaying gains. The same conclusion was reached when increasing the number of maximum transmissions that the hybrid-ARQ protocol can assume. When using one relay, the maximum achievable throughput performance is attained when placing the relay exactly halfway between the source and the destination. Increasing the number of relays results in higher relaying gains though the gains diminish with the addition of more relays. Finally, it was shown that randomly placing the relays results in a poorer performance than with pre-calculated placement. When comparing the unconstrained Gaussian input plots to the QPSK constrained modulation, it can be seen that the modulation constrained curves closely follow that of the unconstrained modulation at low SNR. At high SNR, the behavior is the same, while in the mid-range values there exists a $1 \mathrm{~dB}$ difference between the unconstrained Gaussian input and the QPSK constrained modulation. Hybrid-ARQ based relay networks suffer no diversity losses due to the use of hybrid-ARQ, and provide relaying gains that make relaying quite energy efficient, particularly, for low SNR values. 


\section{Chapter 7}

\section{Conclusions}

\subsection{Summary and Conclusions}

In this thesis, we investigated the impact of modulation constraints on the throughput of hybrid-ARQ in a block fading environment. First, we considered the impact of modulation constraints on information outage probability for a block fading channel with a fixed length codeword for point-to-point links. This was achieved by deriving the capacities for the unconstrained and constrained modulation for incremental redundancy and code combining as well as repetition coding and diversity combining in a block fading channel. The derivations were based on the use of BICM whereby the block fading channel is transformed into an addition of individual Gaussian channels. Finally, a comparison of the effect of different rates on the unconstrained Gaussian input and with modulation constraints was provided. Then, we considered the effect of modulation constraints upon the throughput of hybrid-ARQ, where the rate of the codeword varies depending on the instantaneous channel conditions. These theoretical bounds were compared against the simulated performance of HSDPA, a newly standardized hybrid-ARQ protocol that uses QPSK and 16-QAM bit interleaved turbo-coded modulation. This thesis presented a methodology for determining the information theoretic throughput bound under modulation constraints, thereby allowing the relative throughput losses due to modulation and coding to be separated.

The analysis was extended to encompass hybrid-ARQ based relay networks, where retransmissions can occur from relays that have overheard the source and decoded the message 
successfully. As with the point-to-point case, the impact of modulation constraints on hybridARQ based practical relay networks were also considered. Finally, performance limits and throughput numerical results were presented taking into consideration the effect of path loss parameters and various relay network topologies.

After examining the throughput of practical hybrid-ARQ systems in block fading, it was shown that there is always a loss relative to the information theoretic bounds derived in [3]. From our results, we can conclude that in the case of HSDPA, this loss is in the range of 1-2 dB. While there are several causes for this loss, these causes can be roughly partitioned into those that are due to the modulation constraints and those that are due to the use of a practical code. The results also proved that while modulation constraints cause a loss in diversity in FEC-coded block fading channels, there is no such diversity loss when using hybrid-ARQ. Furthermore, it was shown that the diversity of the system depends on the rate, number of blocks, and the choice of modulation. In hybrid-ARQ based relay networks, higher relaying gains were achieved when employing one or more of the following: higher path loss exponent coefficient, increasing the number of maximum transmissions, and increasing the number of relays. Modulation constraints were not found as a limiting factor for hybrid-ARQ relay networks as the loss is quite acceptable.

\section{2 $\quad$ Future Work}

In this thesis, the focus was mainly on the performance of hybrid-ARQ under modulation constraints. Even though a practical channel code example was given, future work can investigate the performance of other channel codes (e.g. Low Density Parity Check or LDPC codes). The relay network model can be further extended to encompass groups of clusters of nodes. Under this assumption, an investigation of the performance of hybrid-ARQ under modulation constraints can be carried out in order to take into account the interference that is generated by neighboring clusters. The idea of using multiple sources can constitute an interesting area for future work, where now the message is known to more than one node at the beginning of transmission. 


\section{References}

[1] J. A. Gutierrez, M. Naeve, E. Callaway, M. Bourgeois, C. Mitter, and B. Heile, "IEEE 802.15.4: A developing standard for low-power low-cost wireless personal area networks," IEEE Network, vol. 15, pp. 12-19, Sept.-Oct. 2001.

[2] S. Wicker, Error Control Systems for Digital Communications and Storage, Prentice Hall, Inc., Englewood Cliffs, NJ, 1995.

[3] G. Caire and D. Tuninetti, "The throughput of hybrid-ARQ protocols for the Gaussian collision channel," IEEE Trans. Inform. Theory, vol. 47, no. 5, pp. 1971-1988, July 2001.

[4] R. Knopp and P. A. Humblet, "On coding for block fading channels," IEEE Trans. Inform. Theory, vol. 46, no. 1, pp. 189-205, Jan. 2000.

[5] L. Ozarow, S. Shamai, and A. D. Wyner, "Information theoretic considerations for cellular mobile radio," IEEE Trans. Veh. Tech., vol. 43, pp. 359-378, May 1994.

[6] A. G. Fàbregas and G. Caire, "Coded modulation in the block-fading channel: Coding theorems and code construction," IEEE Trans. Inform. Theory, vol. 52, pp. 91-114, Jan. 2006.

[7] T. Ghanim and M.C. Valenti, "The throughput of hybrid-arq in block fading under modulation constraints," in Conf. on Information Sciences and Systems (CISS), Mar 2006.

[8] European Telecommunications Standards Institute, "Universal mobile telecommunications system (UMTS): Multiplexing and channel coding (FDD)," 3GPP TS 25.212 version 6.6.0, release 6, Sept. 2005.

[9] R. Love, A. Ghosh, W. Xiao, and R. Ratasuk, "Performance of 3GPP high speed downlink packet access (HSDPA)," Proc. IEEE Veh. Tech. Conf. (VTC), Sept. 2004.

[10] G. Caire, G. Taricco, and E. Biglieri, "Bit-interleaved coded modulation," IEEE Trans. Inform. Theory, vol. 44, pp. 927-946, May 1998.

[11] B. Zhao and M. C. Valenti, "Practical relay networks: A generalization of hybridARQ," IEEE J. Select. Areas Commun., vol. 23, no. 1, pp. 786-787, Jan. 2005. 
[12] E. C. van der Meulen, "Three-terminal communication channels," Advanced Applied Probability, vol. 3, pp. 120-154, 1971.

[13] T. M. Cover and A. A. El Gamal, "Capacity theorems for the relay channel," IEEE Trans. Inform. Theory, vol. 25, pp. 572-584, Sept. 1979.

[14] J. N. Laneman, Cooperative diversity in wireless networks: Algorithms and architectures, Ph.D. thesis, Massachusetts Institute of Technology, Cambridge, MA, Aug. 2002.

[15] J. N. Laneman, D. N. C. Tse, and G. W. Wornell, "Cooperative diversity in wireless networks: Efficient protocols and outage behavior," IEEE Trans. Inform. Theory, 2004, accepted for publication.

[16] A. Sendonaris, E. Erkip, and B. Aazhang, "User cooperation diversity — part I: System description," IEEE Trans. Commun., vol. 51, no. 11, pp. 1927-1938, Nov. 2003.

[17] B. Sklar, "Rayleigh fading channels in mobile digital communication systems part I: Characterization," IEEE Communications Magazine, vol. 35, no. 9, pp. 136-146, Sept. 1997.

[18] P. A. Bello, "Characterization of randomly time-variant linear channels," IEEE Trans Communications Systems, vol. CS 11, no. 4, pp. 360-393, Dec. 1963.

[19] G. Ungerboeck, "Channel coding with multilevel/phase signals," IEEE Trans. Inform. Theory, vol. IT-28, pp. 56-67, Jan. 1982.

[20] E. Biglieri, Coding for Wireless Channels, Springer Science + Business Media Inc., 2005.

[21] A. J. Viterbi, "An intuitive justification and a simplified implemetation of the MAP decoder for convolutional codes," IEEE J. Select. Areas Commun., vol. 16, no. 2, pp. 260-264, Feb. 1998.

[22] C. E. Shannon, "A mathematical theory of communication," Bell System Technical Journal, July/Oct. 1948.

[23] A. Leon-Garcia, Probability and Random Processes for Electrical Engineers, AddisonWesley Publishing Company, Reading, MA, 1994.

[24] T. M. Cover and J. A. Thomas, Elements of Information Theory, Wiley, 1991.

[25] S. G. Wilson, Digital Modulation and Coding, Prentice Hall, Upper Saddle River, NJ, 1996.

[26] M. C. Valenti, M. Robert, and J. H. Reed, "On the throughput of Bluetooth data transmissions," in IEEE Wireless Commun. and Networking Conf., Orlando, FL, Mar. 2002, pp. 119-123.

[27] S. Kallel, R. Link, and S. Bakthiyari, "Throughput performance of memory ARQ schemes," IEEE Trans. Veh. Tech., vol. 48, no. 3, pp. 891-899, May 1999. 
[28] D. Chase, "Code combining: A maximum-likelihood decoding approach for combining an arbitrary number of noisy packets," IEEE Trans. Commun., vol. 33, pp. 385-393, May 1985.

[29] C. Berrou, A. Glavieux, and P. Thitimasjshima, "Near Shannon limit error-correcting coding and decoding: Turbo-codes(1)," in Proc. IEEE Int. Conf. on Commun. (ICC), Geneva, Switzerland, May 1993, pp. 1064-1070.

[30] P. Elias, "Error free coding," IRE Trans Inform. Theory, vol. IT 4, pp. 29-37, 1954.

[31] G. D. Forney, "Convolutional codes I: Algebraic structure," IEEE Trans. Inform. Theory, vol. IT 16, no. 6, pp. 720-738, Jan. 1970.

[32] A. J. Viterbi, "Error bounds for convolutional codes and an asymptotically optimum decoding algorithm," IEEE Trans. Inform. Theory, vol. 13, no. 2, pp. 260-269, Apr. 1967.

[33] M. C. Valenti and J. Sun, "The UMTS turbo code and an efficient decoder implementation suitable for software defined radios," Int. J. Wireless Info. Networks, vol. 8, pp. 203-216, Oct. 2001.

[34] European Telecommunications Standards Institute, "Universal mobile telecommunications system (UMTS): User equipment (UE) radio transmission and reception (FDD)," 3GPP TS 25.101 version 6.9.0, release 6, Sept. 2005.

[35] S. Dolinar, D. Divsalar, and F. Pollara, "Code performance as a function of block size," JPL TDA Progress Report, Tech. Rep., May 1998.

[36] D. N. Rowitch and L. B. Milstein, "On the performance of hybrid FEC/ARQ systems using rate compatable punctured turbo (RCPT) codes," IEEE Trans. Commun., vol. 48, no. 6, pp. 948-959, June 2000.

[37] M. Luby, "LT codes," Symposium on Foundations of Computer Science, Nov. 2002.

[38] A. Shokrollahi, "Raptor codes," Proc. IEEE Int. Symp. on Inform. Theory (ISIT), July 2004.

[39] S. Dolinar, D. Divsalar, and F. Pollara, "Turbo code performance as a function of code block size," Proc. IEEE Int. Symp. on Inform. Theory (ISIT), Aug 1998.

[40] M. Khojastepour, A. Sabharwal, and B. Aazhang, "On the capacity of 'cheap' relay networks," in Conf. on Information Sciences and Systems (CISS), Baltimore, MD, Mar. 2003.

[41] M. Zorzi and R. R. Rao, "Geographic random forwarding (GeRaF) for ad hoc and sensor networks: Energy and latency performance," IEEE Trans. Mobile Comp., vol. 2, no. 4, pp. 349-365, Oct. 2003.

[42] E. Beres and R. Adve, "On selection cooperation in distributed networks," in Conf. on Information Sciences and Systems (CISS), Mar 2006. 


\section{A Numerical Integration : Information Outage Prob- ability}

Numerical integration of the information outage probability for the case of $1 \leqslant B \leqslant 4$ is derived below for the unconstrained Gaussian input. Recall that the information outage probability is the probability that the instantaneous capacity $C\left(\gamma_{1}, \ldots, \gamma_{B}\right)$ is less than the rate $R$ in bits per symbol,

$$
p_{0}\left(\gamma_{1}, \ldots \gamma_{B}\right)=P\left[C\left(\gamma_{1}, \ldots, \gamma_{B}\right)<R\right]
$$

Recall as well that because the fading coefficient $h[b]$ of the $b^{\text {th }}$ block is constant for the entire duration of the block, the channel during one block is conditionally Gaussian (conditioned on $h[b])$. However, since the fading coefficient is random, then so is the instantaneous SNR of the $b^{t h}$ block, which was denoted $\gamma_{b} \equiv|h[b]|^{2} \mathcal{E}_{s} / N_{0}$, and therefore so is the corresponding capacity $C\left(\gamma_{b}\right)$. Then the capacity for the unconstrained Gaussian input is simply:

$$
\begin{aligned}
C(\gamma) & =\log _{2}\left(1+\gamma_{b}\right) \\
& =\log _{2}\left(1+|h[b]|^{2} \frac{\mathcal{E}_{s}}{N_{0}}\right)
\end{aligned}
$$

For Rayleigh block fading, $|h[b]|$ is Rayleigh and $|h[b]|^{2}$ is exponentially distributed. Using the previously stated assumption that $E\left\{|h[b]|^{2}\right\}=1$, the exponential pdf is then:

$$
p_{|h[b]|^{2}}(\mu)= \begin{cases}e^{-\mu} & \text { for } \mu \geq 0 \\ 0 & \text { otherwise }\end{cases}
$$


Let $\Gamma=\frac{\mathcal{E}_{s}}{N_{0}}$, and $\alpha=|h[b]|^{2}$. Now consider the case when $\mathrm{B}=1, R_{B}=R, \mathrm{~A}-1$ becomes:

$$
\begin{aligned}
p_{0}\left(\gamma_{1}\right) & =P\left[C\left(1+\alpha_{1} \Gamma\right)<R\right] \\
& =P\left[\log _{2}\left(1+\alpha_{1} \Gamma\right)<R\right] \\
& =P\left[\left(1+\alpha_{1} \Gamma\right)<2^{R}\right] \\
& =P\left[\alpha_{1}<\frac{2^{R}-1}{\Gamma}\right] \\
& =\int_{0}^{\frac{2^{R}-1}{\Gamma}} p\left(\alpha_{1}\right) d \alpha_{1} \\
& =\int_{0}^{\frac{2^{R}-1}{\Gamma}} e^{-\alpha_{1}} d \alpha_{1} \\
p_{0}\left(\gamma_{1}\right) & =1-\exp \left\{-\frac{2^{R}-1}{\Gamma}\right\}
\end{aligned}
$$

For the case of $\mathrm{B}=2$ :

$$
\begin{aligned}
p_{0}\left(\gamma_{1}, \gamma_{2}\right) & =P\left[\frac{1}{2}\left(C\left(1+\alpha_{1} \Gamma\right)+C\left(1+\alpha_{2} \Gamma\right)\right)<R\right] \\
& =P\left[\log _{2}\left(1+\alpha_{1} \Gamma\right)+\log _{2}\left(1+\alpha_{2} \Gamma\right)<2 R\right] \\
& =P\left[\left(1+\alpha_{1} \Gamma\right)\left(1+\alpha_{2} \Gamma\right)<2^{2 R}\right] \\
& =P\left[\left(1+\alpha_{1} \Gamma+\alpha_{2} \Gamma+\alpha_{1} \alpha_{2} \Gamma^{2}\right)<2^{2 R}\right] \\
& =P\left[\alpha_{1}<\Gamma^{-1}\left\{\frac{2^{2 R}}{1+\alpha_{2} \Gamma}-1\right\}\right] \\
& =\int_{0}^{\frac{2^{2 R}-1}{\Gamma}} \int_{0}^{\Gamma^{-1}\left\{\frac{2^{2 R}}{1+\alpha_{2} \Gamma}-1\right\}} p\left(\alpha_{1}\right) p\left(\alpha_{2}\right) d \alpha_{1} d \alpha_{2} \\
& =\int_{0}^{\frac{2^{2 R}-1}{\Gamma}} e^{-\alpha_{2}} \int_{0}^{\Gamma^{-1}\left\{\frac{2^{2 R}}{1+\alpha_{2} \Gamma}-1\right\}} e^{-\alpha_{1}} d \alpha_{1} d \alpha_{2} \\
p_{0}\left(\gamma_{1}, \gamma_{2}\right) & =\int_{0}^{\frac{2^{2 R}-1}{\Gamma}} e^{-\alpha_{2}}\left[1-\exp \left\{-\Gamma^{-1}\left[\frac{2^{2 R}}{1+\alpha_{2} \Gamma}-1\right]\right\}\right] d \alpha_{2}
\end{aligned}
$$

NOTE: The step before the last is a resultant of the fact that the random variables are independent. 
For $\mathrm{B}=3$ :

$$
\begin{aligned}
& p_{0}\left(\gamma_{1}, \gamma_{2}, \gamma_{3}\right)=P\left[\frac{1}{3}\left(C\left(1+\alpha_{1} \Gamma\right)+C\left(1+\alpha_{2} \Gamma\right)+C\left(1+\alpha_{3} \Gamma\right)\right)<R\right] \\
& =P\left[\log _{2}\left(1+\alpha_{1} \Gamma\right)+\log _{2}\left(1+\alpha_{2} \Gamma\right) \log _{2}\left(1+\alpha_{3} \Gamma\right)<3 R\right] \\
& =P\left[\left(1+\alpha_{1} \Gamma\right)\left(1+\alpha_{2} \Gamma\right)\left(1+\alpha_{3} \Gamma\right)<2^{3 R}\right] \\
& =P\left[\left(1+\alpha_{1} \Gamma+\alpha_{2} \Gamma+\alpha_{1} \alpha_{2} \Gamma^{2}+\alpha_{3} \Gamma+\alpha_{1} \alpha_{3} \Gamma+\alpha_{2} \alpha_{3} \Gamma^{2}+\alpha_{1} \alpha_{2} \alpha_{3} \Gamma^{3}\right)<2^{3 R}\right] \\
& =P\left[\alpha_{1}<\Gamma^{-1}\left\{\frac{2^{3 R}}{1+\alpha_{2} \Gamma+\alpha_{3} \Gamma+\alpha_{2} \alpha_{3} \Gamma^{2}}-1\right\}\right] \\
& =\int_{0}^{\frac{2^{3 R}-1}{\Gamma}} \int_{0}^{\Gamma^{-1}\left\{\frac{2^{3 R}}{1+\alpha_{2} \Gamma}-1\right\}} \int_{0}^{\Gamma^{-1}\left\{\frac{2^{3 R}}{1+\alpha_{2} \Gamma+\alpha_{3} \Gamma+\alpha_{2} \alpha_{3} \Gamma^{2}}-1\right\}} p\left(\alpha_{1}\right) p\left(\alpha_{2}\right) p\left(\alpha_{3}\right) d \alpha_{1} d \alpha_{2} d \alpha_{3} \\
& =\int_{0}^{\frac{2^{3 R}-1}{\Gamma}} \int_{0}^{\Gamma^{-1}\left\{\frac{2^{3 R}}{1+\alpha_{2} \Gamma}-1\right\}} e^{-\alpha_{2}} e^{-\alpha_{3}} \int_{0}^{\Gamma^{-1}\left\{\frac{2^{3 R}}{1+\alpha_{2} \Gamma+\alpha_{3} \Gamma+\alpha_{2} \alpha_{3} \Gamma^{2}}-1\right\}} e^{-\alpha_{1}} d \alpha_{1} d \alpha_{2} d \alpha_{3} \\
& p_{0}\left(\gamma_{1}, \gamma_{2}, \gamma_{3}\right)=\int_{0}^{\frac{2^{3 R}-1}{\Gamma}} \int_{0}^{\Gamma^{-1}\left\{\frac{2^{3 R}}{1+\alpha_{2} \Gamma}-1\right\}} e^{-\alpha_{2}} e^{-\alpha_{3}} \\
& {\left[1-\exp \left\{-\Gamma^{-1}\left[\frac{2^{3 R}}{1+\alpha_{2} \Gamma+\alpha_{3} \Gamma+\alpha_{2} \alpha_{3} \Gamma^{2}}-1\right]\right\}\right] d \alpha_{2} d \alpha_{3}}
\end{aligned}
$$

Similarly, when $B=4$, the resultant information outage probability is:

$$
\begin{aligned}
p_{0}\left(\gamma_{1}, \gamma_{2}, \gamma_{3}, \gamma_{4}\right)= & \int_{0}^{\frac{2^{4 R}-1}{\Gamma}} \int_{0}^{\Gamma^{-1}\left\{\frac{2^{4 R}}{1+\alpha_{2} \Gamma}-1\right\}} \int_{0}^{\Gamma^{-1}\left\{\frac{2^{3 R}}{1+\alpha_{2} \Gamma+\alpha_{3} \Gamma+\alpha_{2} \alpha_{3} \Gamma^{2}}-1\right\}} e^{-\alpha_{2}} e^{-\alpha_{3}} e^{-\alpha_{4}} \\
& {\left[1-\exp \left\{-\Gamma^{-1}\left[\frac{2^{4 R}}{\beta}-1\right]\right\}\right] d \alpha_{2} d \alpha_{3} d \alpha_{4} }
\end{aligned}
$$

where $\beta=1+\alpha_{2} \Gamma+\alpha_{3} \Gamma+\alpha_{2} \alpha_{3} \Gamma^{2}+\alpha_{4} \Gamma+\alpha_{2} \alpha_{4} \Gamma^{2}+\alpha_{3} \alpha_{4} \Gamma^{2}+\alpha_{2} \alpha_{3} \alpha_{4} \Gamma^{3}$.

Finally, in order to verify the previous results, Fig. (.1) shows a plot for the block values of $\mathrm{B}=1,2$, and 3 for both the numerical integration results derived here and the Monte Carlo results. As can be seen from the figure they provide a perfect match. 


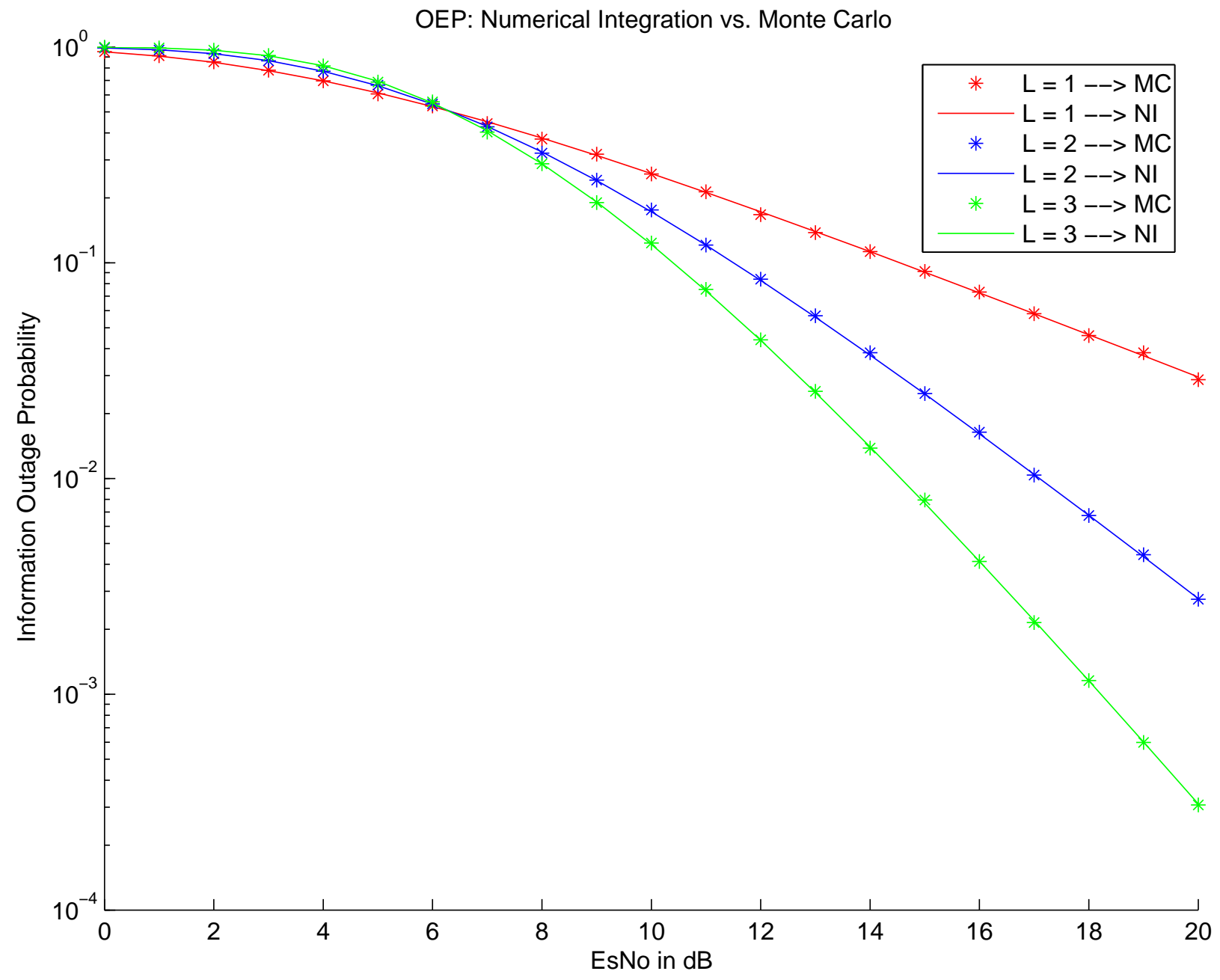

Figure .1: Information outage probability vs SNR in Rayleigh block fading for the unconstrained Gaussian input with rate $R=2$. Numerical integration results (solid line) and Monte Carlo results (star). 


\section{B Maximum Throughput Performance of H-Set 1}

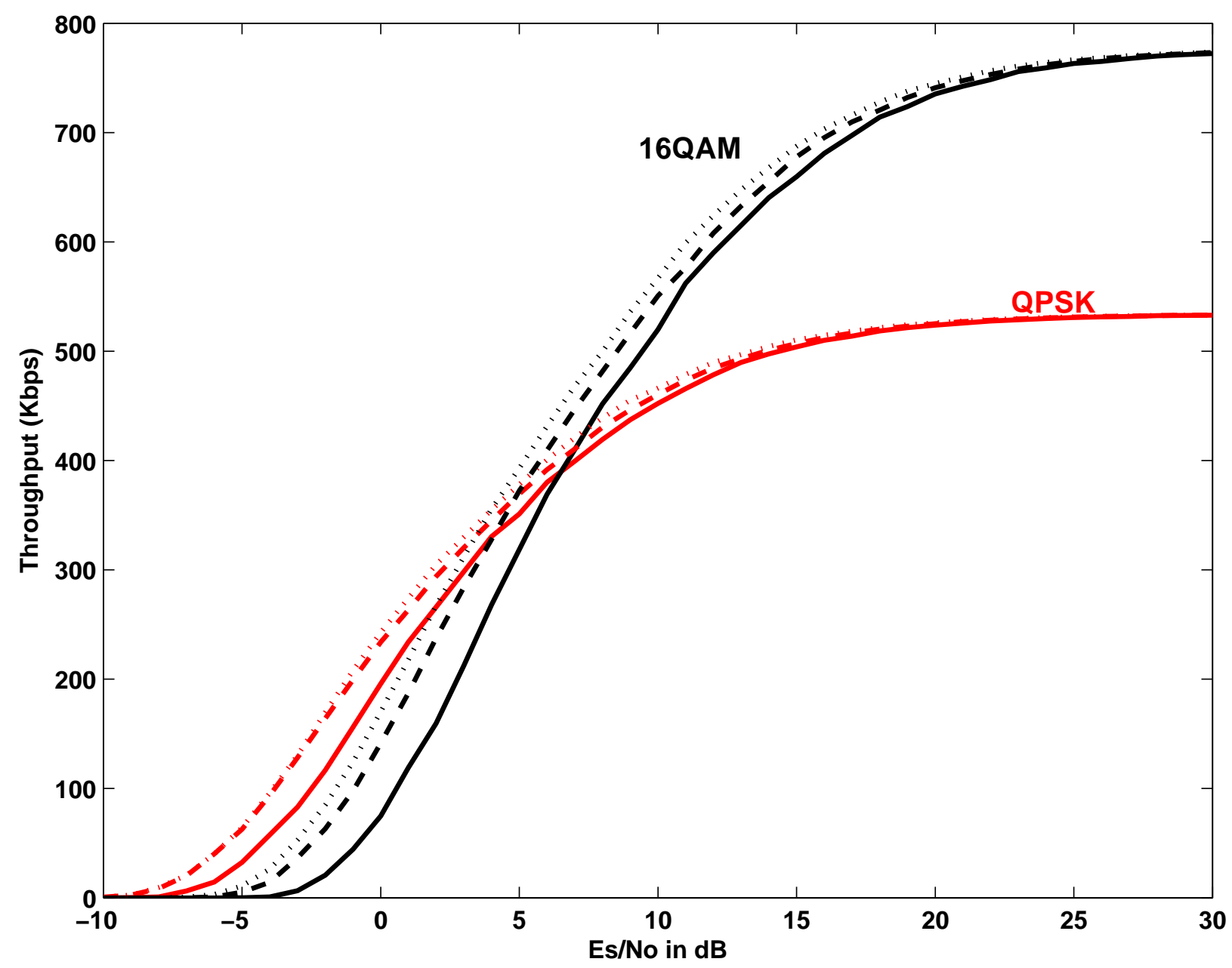

Figure .2: Maximum Throughput of HSDPA H-Set 1 in Rayleigh block fading using QPSK (black) or 16-QAM (red) modulation. For each modulation type, the unconstrained (solid line) and modulation-constrained (dashed line) theoretical limits are compared against the simulated performance of the HSDPA system (dotted line). 